Synthesis of Quinolines, 2-Quinolones, Phenanthridines and 6(5H)-Phenanthridinones via Palladium[0]-Mediated Ullmann Cross-Coupling of 1-Bromo-2-nitroarenes with $\beta$-Halo-enals, -enones or -esters*

Martin G. Banwell, David W. Lupton, Xinghua Ma, Jens Renner and Magne O. Sydnes

Research School of Chemistry, Institute of Advanced Studies, The Australian National University, Canberra, ACT 0200, Australia

$$
\text { mgb@rsc.anu.edu.au }
$$

\title{
Contents Page
}

General S2

Experimental Procedures and Product Characterization for Compounds: 7-8 S3

$\begin{array}{rl}\mathbf{1 0 - 1 1} & \text { S3-S4 } \\ \mathbf{1 3 - 1 4} & \text { S } 4-\mathrm{S} 5 \\ \mathbf{1 6 - 1 9} & \mathrm{S} 5-\mathrm{S} 6 \\ \mathbf{2 1 - 2 2} & \mathrm{S} 6-\mathrm{S} 7 \\ \mathbf{2 4 - 2 7} & \mathrm{S} 7-\mathrm{S} 8 \\ \mathbf{2 9 - 3 2} & \mathrm{S} 8-\mathrm{S} 9 \\ \mathbf{3 4 - 3 5} & \mathrm{S} 9-\mathrm{S} 10 \\ \mathbf{3 7 - 3 8} & \mathrm{S} 10-\mathrm{S} 11 \\ \mathbf{4 0 - 4 1} & \mathrm{S} 11-\mathrm{S} 12 \\ \mathbf{4 3 - 4 4} & \mathrm{S} 12-\mathrm{S} 13 \\ \mathbf{4 6 - 4 7} & \mathrm{S} 13-\mathrm{S} 14 \\ \mathbf{4 9 - 5 0} & \mathrm{S} 14-\mathrm{S} 15 \\ \mathbf{5 2 - 5 3} & \mathrm{S} 15 \\ \mathbf{5 5 - 5 6} & \mathrm{S} 16 \\ \mathbf{5 8 - 5 9} & \mathrm{S} 16-\mathrm{S} 17 \\ \text { References } & \mathrm{S} 18\end{array}$

${ }^{1} \mathrm{H}$ or ${ }^{13} \mathrm{C}$ NMR Spectra of Compounds: $7,10,13,16,18,21$, $23,24,26,29,31,37,38,40,41,43,46,49,52,55,56$ and 58

* The work described here-in is the subject of a patent application submitted to IPAustralia on 17 May 2004. 


\section{Experimental Procedures and Product Characterization}

\section{General Experimental:}

Melting points were measured on a Reichert hot-stage microscope apparatus and are uncorrected. Proton $\left({ }^{1} \mathrm{H}\right)$ and carbon $\left({ }^{13} \mathrm{C}\right)$ NMR spectra were recorded on a Varian Inova 500 spectrometer operating at 500 $\mathrm{MHz}$ for proton and $126 \mathrm{MHz}$ for carbon. In certain cases a Gemini $300 \mathrm{NMR}$ spectrometer, operating at $300 \mathrm{MHz}$ (for proton) and $75 \mathrm{MHz}$ (for carbon), was employed. Spectra were acquired at $20^{\circ} \mathrm{C}$ in deuterochloroform $\left(\mathrm{CDCl}_{3}\right)$ which had been filtered through basic alumina prior to use, or deuteromethanol $\left(\mathrm{CD}_{3} \mathrm{OD}\right)$, deuterium oxide $\left(\mathrm{D}_{2} \mathrm{O}\right)$ or deuterodimethyl sulfoxide $\left[\left(\mathrm{CD}_{3}\right)_{2} \mathrm{SO}\right]$. Signals arising from the residual protio-forms of the solvent were used as the internal standard. Chemical shifts were recorded as $\delta$ values in parts per million (ppm). The assignments of signals observed in the various NMR spectra were often assisted by conducting DEPT, APT, homonuclear $\left({ }^{1} \mathrm{H} /{ }^{1} \mathrm{H}\right)$ correlation spectroscopy (gDQFCOSY), and/or heteronuclear $\left({ }^{1} \mathrm{H} /{ }^{13} \mathrm{C}\right)$ correlation spectroscopy (gHMQC or gHMBC) experiments. Infrared spectra $(v \max )$ were recorded on a Perkin-Elmer 1800 Series FTIR Spectrometer. Samples were analysed as $\mathrm{KBr}$ disks (for solids) or as thin films on $\mathrm{NaCl}$ plates (for oils). Low resolution mass spectra were recorded on a Micromass-Waters LC-ZMD single quadrupole liquid chromatograph-MS or VG Quattro II triple quadrupole MS instrument using electrospray techniques in positive and/or negative ionization mode. Low resolution EI and FAB mass spectra were recorded on an AUTOSPEC spectrometer or a Kratos Analytical Concept ISQ instrument, the latter being located at the University of Tasmania. High resolution mass spectra were acquired by FAB methods on a Thermoquest Mat95XL instrument (located at CSIRO Molecular Science, Melbourne) or by EI methods on an AUTOSPEC instrument. Optical rotations were measured with a Perkin-Elmer 241 polarimeter at the sodium-D line $(589 \mathrm{~nm})$ and the concentrations $(c)(\mathrm{g} / 100 \mathrm{~mL})$ indicated using spectroscopic grade $\mathrm{CHCl}_{3}$ unless otherwise specified. The measurements were carried out in a cell with a path length (l) of 1 dm. Specific rotations $[\alpha]_{D}$ were calculated using the equation $[\alpha]_{D}=100 . \alpha /(c .1)$ and are given in $10^{-1} \cdot \mathrm{deg} \cdot \mathrm{cm}^{2} \cdot \mathrm{g}^{-1}$. Elemental analyses were performed by the Australian National University's Microanalytical Services Unit based at the Research School of Chemistry, Canberra, Australia. The unit cell parameters were recorded on a Nonius Kappa CCD instrument. Dichloromethane and chloroform were distilled from calcium hydride and THF and benzene were distilled, under nitrogen, from sodium benzophenone ketyl. Where necessary, reactions were performed under a nitrogen atmosphere. 
(Z)- $\beta$-(2'-Nitrophenyl)cinnamaldehyde (7). A solution of 1-bromo-2-nitrobenzene (1, R=H) $(396$ $\mathrm{mg}, 2 \mathrm{mmol})$ and (Z)- $\beta$-bromocinnamaldehyde (6) $(420 \mathrm{mg}, 2 \mathrm{mmol})$ in DMSO $(6 \mathrm{~mL})$ was treated with $\mathrm{Pd}_{2}(\mathrm{dba})_{3}(60 \mathrm{mg}, 3 \mathrm{~mol} \%)$ and copper powder $(630 \mathrm{mg}, 0.01 \mathrm{~g}$.-atom). The ensuing mixture was heated at $80{ }^{\circ} \mathrm{C}$ (oil bath) with vigorous stirring for $2 \mathrm{~h}$ then cooled and diluted with diethyl ether $(50 \mathrm{~mL})$. The resulting mixture was filtered through a pad of Celite ${ }^{\mathrm{TM}}$ and the solids thus retained were washed with diethyl ether $(5 \times 10 \mathrm{~mL})$. The combined filtrates were washed with water $(3 \times 20 \mathrm{~mL})$ and brine $(1 \times 30$ $\mathrm{mL})$ then dried $\left(\mathrm{MgSO}_{4}\right)$, filtered and concentrated under reduced pressure to give a yellow oil. Subjection of this material to flash chromatography (4:1 $\mathrm{v} / \mathrm{v}$ hexane-ethyl acetate elution) and concentration of the appropriate fractions $\left(R_{\mathrm{f}}=0.2\right)$ afforded compound $7(310 \mathrm{mg}, 68 \%)$ as a yellow crystalline solid, $\mathrm{mp}=$ 114-121 ${ }^{\circ} \mathrm{C} .{ }^{1} \mathrm{H}$ NMR $\left(\mathrm{CDCl}_{3}, 300 \mathrm{MHz}\right) \delta 9.42(\mathrm{~d}, J=8.1 \mathrm{~Hz}, 1 \mathrm{H}), 8.22(\mathrm{dd}, J=1.5$ and $8.1 \mathrm{~Hz}, 1 \mathrm{H})$, $7.78(\mathrm{dt}, J=1.2$ and $7.2 \mathrm{~Hz}, 1 \mathrm{H}), 7.68(\mathrm{dt}, J=1.5$ and $8.1 \mathrm{~Hz}, 1 \mathrm{H}), 7.48(\mathrm{dd}, J=1.5$ and $7.5 \mathrm{~Hz}, 1 \mathrm{H})$, 7.26-7.39 (m, 5H), $6.70(\mathrm{~d}, J=8.1 \mathrm{~Hz}, 1 \mathrm{H}) ;{ }^{13} \mathrm{C} \mathrm{NMR}\left(\mathrm{CDCl}_{3}, 75 \mathrm{MHz}\right) \delta 191.5,158.4,148.1,137.1$, 133.5, 133.1, 131.9, 130.8, 130.3, 128.8, 127.2, 126.8, 125.3; IR $v_{\text {max }}(\mathrm{KBr}) 3063,2847,2753,1670,1595$, 1572, 1526, 1447, 1346, 1234, 1132, 1077, $855 \mathrm{~cm}^{-1}$; MS, $m / z$ (EI, 70eV) $253\left(\mathrm{M}^{+*}, 13 \%\right), 233$ (4), 224 (100), 196 (92), 180 (55), 178 (53), 167 (50), 165 (47), 152 (42), 77 (52); HRMS, Found: $\mathrm{M}^{+*}, 253.0741$ $\mathrm{C}_{15} \mathrm{H}_{11} \mathrm{NO}_{3}$ requires $\mathrm{M}^{+\bullet}, 253.0739$.

4-Phenylquinoline (8). Commercially available titanium(III) chloride tetrahydrofuran complex (1.68 g, $4 \mathrm{mmol})$ was dissolved in water $(3 \mathrm{~mL})$ then ammonium acetate $(6 \mathrm{~mL}$ of a $2.5 \mathrm{M}$ aqueous solution) and acetone $(3 \mathrm{~mL})$ were added. The resulting dark-blue and biphasic mixture was stirred at $18{ }^{\circ} \mathrm{C}$ for 10 minutes then a solution of compound $7(127 \mathrm{mg}, 0.5 \mathrm{mmol})$ in acetone $(6 \mathrm{~mL})$ was added dropwise. After $0.25 \mathrm{~h}$ the now pale-blue reaction mixture was diluted with water $(100 \mathrm{~mL})$ and extracted with ethyl acetate $(3 \times 75 \mathrm{~mL})$. The combined extracts were washed with sodium bicarbonate $(3 \times 75 \mathrm{~mL}$ of a saturated aqueous solution) then brine $(3 \times 75 \mathrm{~mL})$ before being dried $\left(\mathrm{MgSO}_{4}\right)$, filtered and concentrated under reduced pressure to give a light-yellow oil. Subjection of this material to flash chromatography (80:20:1 $\mathrm{v} / \mathrm{v} / \mathrm{v}$ hexane-ethyl acetate-triethylamine elution) and concentration of the appropriate fractions $\left(R_{\mathrm{f}}=0.2\right)$ afforded compound $\mathbf{8}^{2}(51 \%)$ as an opaque oil (lit. $\left.{ }^{2} \mathrm{mp}=61-62{ }^{\circ} \mathrm{C}\right) .{ }^{1} \mathrm{H} \mathrm{NMR}\left(\mathrm{CDCl}_{3}, 300 \mathrm{MHz}\right) \delta 8.94$ (d, $J=4.5 \mathrm{~Hz}, 1 \mathrm{H}), 8.18(\mathrm{dd}, J=1.2$ and $8.7 \mathrm{~Hz}, 1 \mathrm{H}), 7.92(\mathrm{~d}, J=7.8 \mathrm{~Hz}, 1 \mathrm{H}), 7.72$ (ddd, $J=1.2,6.9$ and $9.9 \mathrm{~Hz}, 1 \mathrm{H}), 7.54-7.46(\mathrm{~m}, 6 \mathrm{H}), 7.34(\mathrm{~d}, J=4.5 \mathrm{~Hz}, 1 \mathrm{H}) ;{ }^{13} \mathrm{C} \mathrm{NMR}\left(\mathrm{CDCl}_{3}, 75 \mathrm{MHz}\right) \delta 149.9,148.6$, $148.5,137.9,129.8,129.5,129.3,128.5,128.4,126.6,125.8,121.3,125.3$; IR $v_{\max }(\mathrm{KBr}) 3058,1584$, 1508, 1490, 1444, 1418, 1389, 850, 768, 698, $611 \mathrm{~cm}^{-1}$; MS, $\mathrm{m} / \mathrm{z}$ (EI, 70eV) $205\left(\mathrm{M}^{+*}, 100 \%\right), 204$ (93), $176(28), 102(23), 88(15)$.

2-(2'-Nitrophenyl)cyclopent-1-enecarbaldehyde (10). Cross-coupling of 1-bromo-2-nitrobenzene (1, $\mathrm{R}=\mathrm{H}$ ) with 2-bromocyclopent-1-enecarboxaldehyde $(\mathbf{9})^{1}$ in the same manner as described above for the preparation of compound $\mathbf{7}$ gave an oily solid on work-up. Subjection of this material to flash 
chromatography ( $4: 1 \mathrm{v} / \mathrm{v}$ hexane-ethyl acetate elution) and concentration of the appropriate fractions $\left(R_{\mathrm{f}}=\right.$ $0.3)$ afforded compound $\mathbf{1 0}(82 \%)$ as a yellow crystalline solid, $\mathrm{mp}=105-115^{\circ} \mathrm{C} .{ }^{1} \mathrm{H}$ NMR $\left(\mathrm{CDCl}_{3}, 300\right.$ MHz) $\delta 9.45(\mathrm{~s}, 1 \mathrm{H}), 8.13(\mathrm{dd}, J=1.2$ and $8.1 \mathrm{~Hz}, 1 \mathrm{H}), 7.67(\mathrm{dt}, J=1.5$ and $7.5 \mathrm{~Hz}, 1 \mathrm{H}), 7.56(\mathrm{dt}, J=1.2$ and $7.5 \mathrm{~Hz}, 1 \mathrm{H}), 7.32(\mathrm{dd}, J=1.5$ and $7.5 \mathrm{~Hz}, 1 \mathrm{H}), 2.85$ (broad t $J=7.5 \mathrm{~Hz}, 2 \mathrm{H}), 2.71$ (tt, $J=2.1$ and 7.5 $\mathrm{Hz}, 2 \mathrm{H}), 2.07(\mathrm{p}, J=7.5 \mathrm{~Hz}, 2 \mathrm{H}) ;{ }^{13} \mathrm{C} \mathrm{NMR}\left(\mathrm{CDCl}_{3}, 75 \mathrm{MHz}\right) \delta 188.5,160.1,147.5,141.0,133.4$, 131.2(0), 131.1(8), 129.5, 125.0, 40.2, 30.1, 22.4; IR $v_{\max }$ (neat) 2949, 2855, 1671, 1602, 1570, 1526, 1346, 1302, 1237, 847, 789, $746 \mathrm{~cm}^{-1}$; MS, m/z (EI, 70eV) 218 (M+*, 37\%), 188 (10), 131 (20), 89 (100), 88 (57), 73 (46), 70 (69), 61 (86); HRMS, Found: $(\mathrm{M}+\mathrm{H})^{+}$, 218.0814. $\mathrm{C}_{12} \mathrm{H}_{11} \mathrm{NO}_{3}$ requires $(\mathrm{M}+\mathrm{H})^{+}$, 218.0817.

2,3-Dihydro-1H-cyclopenta[c]quinoline (11). A magnetically stirred solution of compound $\mathbf{1 0}$ (100 $\mathrm{mg}, 0.46 \mathrm{mmol})$ in methanol $(20 \mathrm{~mL})$ maintained at $18^{\circ} \mathrm{C}$ was treated with $10 \%$ palladium on carbon $(23$ $\mathrm{mg}$ ) and the resulting mixture exposed to an atmosphere of dihydrogen (1 atm.) while being protected from light. After $10 \mathrm{~h}$ the reaction mixture was filtered through a pad of Celite ${ }^{\mathrm{TM}}$ and the solids thus retained washed with methanol $(20 \mathrm{~mL})$. The combined filtrates were concentrated under reduced pressure to give a light-yellow oil. Subjection of this material to flash chromatography (80:20:1 v/v hexane-ethyl acetate-triethylamine elution) and concentration of the appropriate fractions $\left(R_{\mathrm{f}}=0.3\right)$ afforded compound $\mathbf{1 1}^{3}(92 \%)$ as a pale-yellow crystalline solid, $\mathrm{mp}=45-52{ }^{\circ} \mathrm{C}\left(\right.$ lit. $\left.{ }^{3} \mathrm{mp}=58-59{ }^{\circ} \mathrm{C}\right) .{ }^{1} \mathrm{H}$ NMR $\left(\mathrm{CDCl}_{3}, 300\right.$ MHz) $\delta 8.80(\mathrm{~s}, 1 \mathrm{H}), 8.10(\mathrm{~d}, J=8.4 \mathrm{~Hz}, 1 \mathrm{H}), 7.76($ broad d, $J=7.8 \mathrm{~Hz}, 1 \mathrm{H}), 7.63($ broad t, $J=8.4 \mathrm{~Hz}$, $1 \mathrm{H}), 7.50$ (broad t, $J=7.8 \mathrm{~Hz}, 1 \mathrm{H}), 3.23(\mathrm{t}, J=7.5 \mathrm{~Hz}, 2 \mathrm{H}), 3.10(\mathrm{t}, J=7.5 \mathrm{~Hz}, 2 \mathrm{H}), 2.50(\mathrm{p}, J=7.5 \mathrm{~Hz}$, $2 \mathrm{H}) ;{ }^{13} \mathrm{C} \mathrm{NMR}\left(\mathrm{CDCl}_{3}, 75 \mathrm{MHz}\right) \delta 149.9,147.6,146.7,136.6,129.5,128.2,126.3,126.0,124.1,31.2$, 30.8, 24.3; IR $v_{\text {max }}(\mathrm{KBr}) 3298,2937,2856,1646,1587,1568,1508,1461,1435,1402,1310,1295,1152$, 1141, $756 \mathrm{~cm}^{-1}$; MS, m/z (EI, 70eV) 169 (M+*, 40\%), 168 (35), 167 (10), 115 (12), 65 (30), 63 (100), 62 (42), 58 (50); HRMS, Found: $\mathrm{M}^{+*}, 169.0888 . \mathrm{C}_{12} \mathrm{H}_{11} \mathrm{~N}$ requires $\mathrm{M}^{+*}, 169.0891$.

2-(2'-Nitrophenyl)cyclohex-1-enecarbaldehyde (13). Cross-coupling of 1-bromo-2-nitrobenzene (1, $\mathrm{R}=\mathrm{H}$ ) with 2-bromocyclohex-1-enecarboxaldehyde $(\mathbf{1 2})^{1}$ in the same manner as described above for the preparation of compound $\mathbf{7}$ gave an oily solid on work-up. Subjection of this material to flash chromatography $\left(9: 1 \mathrm{v} / \mathrm{v}\right.$ hexane-ethyl acetate elution) and concentration of the appropriate fractions $\left(R_{\mathrm{f}}=\right.$ $0.2)$ afforded compound $\mathbf{1 3}(82 \%)$ as a yellow crystalline solid, $\mathrm{mp}=55-58{ }^{\circ} \mathrm{C} .{ }^{1} \mathrm{H}$ NMR $\left(\mathrm{CDCl}_{3}, 300\right.$ MHz) $\delta 9.22(\mathrm{~s}, 1 \mathrm{H}), 8.08(\mathrm{dd}, J=1.2$ and $8.1 \mathrm{~Hz}, 1 \mathrm{H}), 7.62(\mathrm{dt}, J=1.2$ and $7.5 \mathrm{~Hz}, 1 \mathrm{H}), 7.50(\mathrm{dt}, J=1.2$ and $8.1 \mathrm{~Hz}, 1 \mathrm{H}), 7.28(\mathrm{dd}, J=1.2$ and $7.5 \mathrm{~Hz}, 1 \mathrm{H}), 2.50(\mathrm{~m}, 1 \mathrm{H}), 2.42-2.16$ (complex m, 3H), 1.84-1.70 (complex m, 3H), $1.60(\mathrm{~m}, 1 \mathrm{H}) ;{ }^{13} \mathrm{C} \mathrm{NMR}\left(\mathrm{CDCl}_{3}, 75 \mathrm{MHz}\right) \delta 191.3,156.3,147.2,135.4,135.1,133.3$, 131.0, 129.0, 124.8, 33.6, 22.1, 21.7, 21.0; IR $v_{\max }$ (neat) 2931, 2867, 1670, 1630, 1606, 1570, 1522, 1345, 1273, 1212, 980, 855, 787, $747 \mathrm{~cm}^{-1}$; MS, $\mathrm{m} / \mathrm{z}$ (EI, 70eV) $232\left[(\mathrm{M}+\mathrm{H})^{+}, 1 \%\right), 202(40), 186(32), 174$ (35), 158 (100), 146 (55), 144 (39), 132 (97), 130 (95), 128 (65), 115 (80), 91 (58), 77 (75); HRMS, Found: $(\mathrm{M}+\mathrm{H})^{+}, 232.0972 \mathrm{C}_{13} \mathrm{H}_{13} \mathrm{NO}_{3}$ requires $(\mathrm{M}+\mathrm{H})^{+}, 232.0974$. 
7,8,9,10-Tetrahydrophenanthridine (14). Reductive cyclization of compound 13 in the same manner as described above for the preparation of compound $\mathbf{1 1}$ afforded a light-yellow oil on work-up. Subjection of this material to flash chromatography $(80: 20: 1 \mathrm{v} / \mathrm{v} / \mathrm{v}$ hexane-ethyl acetate-triethylamine elution) and concentration of the appropriate fractions $\left(R_{\mathrm{f}}=0.15\right)$ afforded compound $14^{2}(82 \%)$ as a pale-yellow crystalline solid, $\mathrm{mp}=52-58{ }^{\circ} \mathrm{C}\left(\right.$ lit. $\left.^{2} \mathrm{mp}=61-62{ }^{\circ} \mathrm{C}\right) .{ }^{1} \mathrm{H}$ NMR $\left(\mathrm{CDCl}_{3}, 300 \mathrm{MHz}\right) \delta 8.62(\mathrm{~s}, 1 \mathrm{H}), 8.06$ $(\mathrm{d}, J=8.4 \mathrm{~Hz}, 1 \mathrm{H}), 7.92(\mathrm{~d}, J=8.4 \mathrm{~Hz}, 1 \mathrm{H}), 7.63(\mathrm{t}, J=8.4 \mathrm{~Hz}, 1 \mathrm{H}), 7.53(\mathrm{t}, J=8.4 \mathrm{~Hz}, 1 \mathrm{H}), 3.11(\mathrm{t}, J=$ $6.3 \mathrm{~Hz}, 2 \mathrm{H}), 2.90(\mathrm{t}, J=6.3 \mathrm{~Hz}, 2 \mathrm{H}), 1.93(\mathrm{~m}, 4 \mathrm{H}) ;{ }^{13} \mathrm{C} \mathrm{NMR}\left(\mathrm{CDCl}_{3}, 75 \mathrm{MHz}\right) \delta 152.3,146.2,141.4$, 129.7, 129.6, 128.0, 127.6, 126.3, 122.6, 27.1, 24.9, 22.4, 22.3; IR $v_{\max }$ (KBr) 2932, 1714, 1591, 1573, $1505,1434,1387,1337,1216,1162,1127,909,755 \mathrm{~cm}^{-1}$; MS, $m / z$ (EI, 70eV) $183\left(\mathrm{M}^{+*}, 100 \%\right), 182$ (75), 168 (39), 167 (42), 155 (75), 154 (50), 115 (35).

2-(2'-Nitrophenyl)cyclohept-1-enecarbaldehyde (16). Cross-coupling of 1-bromo-2-nitrobenzene (1, $\mathrm{R}=\mathrm{H}$ ) with 2-bromocyclohept-1-enecarboxaldehyde $(\mathbf{1 5})^{1}$ in the same manner as described above for the preparation of compound $\mathbf{7}$ gave yellow solid on work-up. Subjection of this material to flash chromatography ( $4: 1 \mathrm{v} / \mathrm{v}$ hexane-ethyl acetate elution) and concentration of the appropriate fractions $\left(R_{\mathrm{f}}=\right.$ 0.35) afforded compound $16(75 \%)$ as a yellow crystalline solid, $\mathrm{mp}=98-102{ }^{\circ} \mathrm{C} .{ }^{1} \mathrm{H}$ NMR $\left(\mathrm{CDCl}_{3}, 300\right.$ MHz) $\delta 9.21(\mathrm{~s}, 1 \mathrm{H}), 8.15(\mathrm{dd}, J=1.5$ and $8.1 \mathrm{~Hz}, 1 \mathrm{H}), 7.63(\mathrm{dt}, J=1.5$ and $7.5 \mathrm{~Hz}, 1 \mathrm{H}), 7.53(\mathrm{dt}, J=1.5$ and $7.5 \mathrm{~Hz}, 1 \mathrm{H}), 7.24(\mathrm{dd}, J=1.5$ and $7.5 \mathrm{~Hz}, 1 \mathrm{H}), 2.93-2.77(\mathrm{~m}, 2 \mathrm{H}), 2.40(\mathrm{~m}, 2 \mathrm{H}), 1.99-1.68$ (complex $\mathrm{m}, 4 \mathrm{H}), 1.58-1.30$ (complex m, 2H); ${ }^{13} \mathrm{C}$ NMR $\left(\mathrm{CDCl}_{3}, 75 \mathrm{MHz}\right) \delta$ 191.0, 162.4, 146.5, 141.3, 136.6, 133.4, 131.4, 129.1, 125.1, 38.4, 31.9, 25.7, 25.4, 24.0; IR $v_{\text {max }}(\mathrm{KBr}) 2924,2852,1671,1623,1605,1570$, 1526, 1449, 1344, $749 \mathrm{~cm}^{-1}$; MS, m/z (EI, 70eV) 245 (M+*, 10\%), 216 (40), 197 (38), 172 (100), 146 (90), 132 (95), 130 (91), 115 (81), 77 (84); HRMS, Found: $\mathrm{M}^{+\bullet}, 245.1046 \mathrm{C}_{14} \mathrm{H}_{15} \mathrm{NO}_{3}$ requires $\mathrm{M}^{+*}, 245.1052$.

8,9,10,11-Tetrahydro-7H-cyclohepta[c]quinoline (17). Reductive cyclization of compound 16 in the same manner as described above for the preparation of compound $\mathbf{1 1}$ afforded a light-yellow oil on workup. Subjection of this material to flash chromatography (70:30:1 v/v hexane-ethyl acetate-triethylamine elution) and concentration of the appropriate fractions $\left(R_{\mathrm{f}}=0.35\right)$ afforded compound $\mathbf{1 7}^{4}(84 \%)$ as an orange crystalline solid, $\mathrm{mp}=65-67{ }^{\circ} \mathrm{C}\left(\right.$ lit. $\left.^{4} \mathrm{mp}=67{ }^{\circ} \mathrm{C}\right) .{ }^{1} \mathrm{H} \mathrm{NMR}\left(\mathrm{CDCl}_{3}, 300 \mathrm{MHz}\right) \delta 8.78(\mathrm{~d}, J=$ $9.3 \mathrm{~Hz}, 1 \mathrm{H}), 8.40(\mathrm{~s}, 1 \mathrm{H}), 8.07$ (d, $J=9.3 \mathrm{~Hz}, 1 \mathrm{H}), 7.62-7.56$ (complex m, 2H), $3.18(\mathrm{~m}, 2 \mathrm{H}), 2.87$ (m, $2 \mathrm{H}), 1.02(\mathrm{~m}, 2 \mathrm{H}), 1.69(\mathrm{~m}, 4 \mathrm{H}) ;{ }^{13} \mathrm{C} \mathrm{NMR}\left(\mathrm{CDCl}_{3}, 75 \mathrm{MHz}\right) \delta$ 139.5, 136.3(4), 136.2(6), 128.9, 128.7, 128.2, 123.8, 120.1, 33.2, 31.9, 27.7, 27.0, 26.3; IR $v_{\text {max }}(\mathrm{KBr}) 2923,2852,1627,1387,1307,1187,1146$,

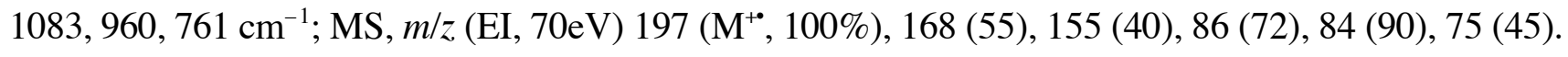

2-(4'-Methoxy-2'-nitrophenyl)cyclohex-1-enecarbaldehyde (18). Cross-coupling of 1-bromo-4methoxy-2-nitrobenzene $(\mathbf{1}, \mathrm{R}=\mathrm{OMe})$ with 2-bromocyclohex-1-enecarbox-aldehyde $(\mathbf{1 2})^{1}$ in the same manner as described above for the preparation of compound $\mathbf{7}$ gave a yellow oil on work-up. Subjection of 
this material to flash chromatography $(7: 3 \mathrm{v} / \mathrm{v}$ hexane-ethyl acetate elution) and concentration of the appropriate fractions $\left(R_{\mathrm{f}} 0.35\right)$ afforded compound $18(88 \%)$ as a yellow oil. ${ }^{1} \mathrm{H} \mathrm{NMR}\left(\mathrm{CDCl}_{3}, 300 \mathrm{MHz}\right)$

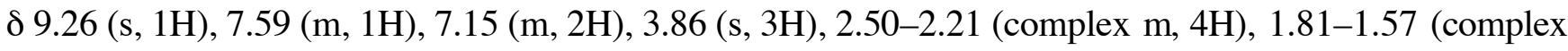
$\mathrm{m}, 4 \mathrm{H}) ;{ }^{13} \mathrm{C} \mathrm{NMR}\left(\mathrm{CDCl}_{3}, 75 \mathrm{MHz}\right) \delta 191.6,159.6,156.4,147.9,135.8,131.9,127.1,119.8,109.5,55.8$, 33.8, 22.2, 21.9, 21.1; IR $v_{\max }(\mathrm{KBr}) 2937,2841,1673,1631,1563,1531,1460,1349,1302,1035,1275$, 1255, 1224, $799 \mathrm{~cm}^{-1}$; MS, m/z (EI, 70eV) $261\left(\mathrm{M}^{+*}, 1 \%\right), 232$ (40), $216(45), 215$ (60), 204 (45), 188 (100), 176 (57), 162 (85), 160 (75), 115 (60), 77 (47); HRMS, Found: $\mathrm{M}^{+\bullet}, 261.0997 \mathrm{C}_{14} \mathrm{H}_{15} \mathrm{NO}_{4}$ requires $\mathrm{M}^{+\bullet}, 261.1001$.

3-Methoxy-7,8,9,10-tetrahydrophenanthridine (19). Reductive cyclization of compound 18 in the same manner as described above for the preparation of compound $\mathbf{1 1}$ afforded a light-yellow oil on workup. Subjection of this material to flash chromatography $(75: 25: 1 \mathrm{v} / \mathrm{v}$ hexane-ethyl acetate-triethylamine elution) and concentration of the appropriate fractions $\left(R_{\mathrm{f}}=0.2\right)$ afforded compound $\mathbf{1 9}^{5}(68 \%)$ as an orange crystalline solid, $\mathrm{mp}=75-78^{\circ} \mathrm{C}\left(\right.$ lit. $\left.^{5} \mathrm{mp}=53-54.7{ }^{\circ} \mathrm{C}\right) .{ }^{1} \mathrm{H} \mathrm{NMR}\left(\mathrm{CDCl}_{3}, 300 \mathrm{MHz}\right) \delta 8.26(\mathrm{~s}$, 1H), $8.04(\mathrm{~s}, 1 \mathrm{H}), 7.79$ (d, $J=8.7 \mathrm{~Hz}, 1 \mathrm{H}), 7.22(\mathrm{~d}, J=8.7 \mathrm{~Hz}, 1 \mathrm{H}), 3.95(\mathrm{~s}, 3 \mathrm{H}), 2.98(\mathrm{t}, J=6.3 \mathrm{~Hz}, 2 \mathrm{H})$, $2.73(\mathrm{t}, J=6.3 \mathrm{~Hz}, 2 \mathrm{H}), 1.92-1.80$ (complex m, 4H); ${ }^{13} \mathrm{C} \mathrm{NMR}\left(\mathrm{CDCl}_{3}, 75 \mathrm{MHz}\right) \delta 160.7,140.5,136.9$, 133.6, 127.9, 124.6, 124.4, 121.1, 98.6, 55.8, 26.7, 24.7, 22.1, 22.0; IR $v_{\max }(\mathrm{KBr}) 2936,1625,1589,1573$, 1512, 1421, 1392, 1281, 1232, 1178, 1029, $733 \mathrm{~cm}^{-1}$; MS, m/z (EI, 70eV) $213\left(\mathrm{M}^{+\bullet}, 60 \%\right), 200$ (25), 199 (25), 185 (35), 115 (25), 86 (77), 84 (100); HRMS, Found: $\mathrm{M}^{+\bullet}, 213.1154 . \mathrm{C}_{14} \mathrm{H}_{15} \mathrm{NO}$ requires $\mathrm{M}^{+\bullet}$, 213.1154 .

(E)-2-(2'-Nitrobenzylidene)cyclohexanone (21). A magnetically stirred mixture of (E)-2(bromomethylene)cyclohexanone (20 $)^{6}(234 \mathrm{mg}, 1.24 \mathrm{mmol})$ and copper(I) iodide (283 $\left.\mathrm{mg}, 1.49 \mathrm{mmol}\right)$ in DMSO $(8 \mathrm{~mL})$ maintained at $70{ }^{\circ} \mathrm{C}$ was treated with 1-bromo-2-nitrobenzene $(\mathbf{1}, \mathrm{R}=\mathrm{H})(500 \mathrm{mg}, 2.48$ $\mathrm{mmol})$, copper powder $(315 \mathrm{mg}, 4.96 \mathrm{mmol})$ and $\mathrm{PdCl}_{2}(\mathrm{dppf})_{2} \mathrm{CH}_{2} \mathrm{Cl}_{2}(25 \mathrm{mg})$. The resulting mixture was heated at $90{ }^{\circ} \mathrm{C}$ (oil bath) for $2 \mathrm{~h}$, cooled to $18{ }^{\circ} \mathrm{C}$ and diluted with $\mathrm{CH}_{2} \mathrm{Cl}_{2}(60 \mathrm{~mL})$ then ammonium chloride (120 mL of a saturated aqueous solution). The separated organic phase was washed with ammonium chloride $(2 \times 100 \mathrm{~mL}$ of a saturated aqueous solution $)$ then dried $\left(\mathrm{MgSO}_{4}\right)$, filtered and concentrated under reduced pressure to give a yellow oil. Subjection of this material to flash chromatography $(100: 3 \rightarrow 100: 10 \mathrm{v} / \mathrm{v}$ hexane-ethyl acetate gradient elution) and concentration of the appropriate fractions $\left(R_{\mathrm{f}}=0.4\right.$ in 55:25:10 v/v/v hexane- $\mathrm{CH}_{2} \mathrm{Cl}_{2}$-ethyl acetate) afforded compound 21 (204 $\mathrm{mg}, 71 \%)$ as a light-yellow solid, $\mathrm{mp}=102-104{ }^{\circ} \mathrm{C} .{ }^{1} \mathrm{H} \mathrm{NMR}\left(\mathrm{CDCl}_{3}, 300 \mathrm{MHz}\right) \delta 8.11(\mathrm{dd}, J=1.2$ and $8.2 \mathrm{~Hz}, 1 \mathrm{H}), 7.63(\mathrm{dt}, J=1.2$ and $7.7 \mathrm{~Hz}, 1 \mathrm{H}), 7.59$ (broad s, $1 \mathrm{H}), 7.49$ (dt, $J=1.2$ and $8.2 \mathrm{~Hz}, 1 \mathrm{H}), 7.32$ $(\mathrm{d}, J=7.7 \mathrm{~Hz}, 1 \mathrm{H}), 2.60-2.44$ (complex m, 4H), $1.93(\mathrm{~m}, 2 \mathrm{H}), 1.73(\mathrm{~m}, 2 \mathrm{H}) ;{ }^{13} \mathrm{C} \mathrm{NMR}\left(\mathrm{CDCl}_{3}, 75 \mathrm{MHz}\right)$

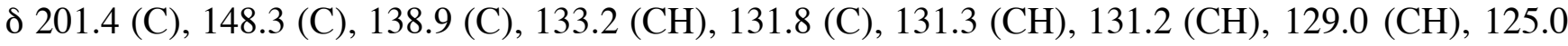
$(\mathrm{CH}), 40.7\left(\mathrm{CH}_{2}\right), 28.8\left(\mathrm{CH}_{2}\right), 24.0\left(\mathrm{CH}_{2}\right), 23.8\left(\mathrm{CH}_{2}\right)$; IR $v_{\max }(\mathrm{KBr}) 2937,2864,1690,1602,1524$, 
1344, 1142, $717 \mathrm{~cm}^{-1}$; MS, m/z (EI, 70eV) $231\left(\mathrm{M}^{+\bullet}, 2 \%\right), 215$ (8), 199 (44), 185 (25), 120 (65), 119 (100), 92 (50), 77 (27), 55 (35); HRMS, Found: $\mathrm{M}^{+\bullet}$, 231.0892. $\mathrm{C}_{13} \mathrm{H}_{13} \mathrm{NO}_{3}$ requires $\mathrm{M}^{+\bullet}, 231.0895$.

1,2,3,4-Tetrahydroacridine (22). Reductive cyclization of compound 21 in the same manner as described above for the preparation of compound 11, save for running the reaction for only $2 \mathrm{~h}$, afforded a light-yellow oil on work-up. Subjection of this material to flash chromatography $(100: 5 \rightarrow 100: 25 \mathrm{v} / \mathrm{v}$ hexane-ethyl acetate gradient elution) and concentration of the appropriate fractions $\left(R_{\mathrm{f}}=0.3\right.$ in 55:25:20 v/v/v hexane- $\mathrm{CH}_{2} \mathrm{Cl}_{2}$-ethyl acetate) afforded compound $\mathbf{2 2}^{7}(46 \%)$ as a light-yellow solid, $\mathrm{mp}=53-55^{\circ} \mathrm{C}$ (lit. $\left.{ }^{7} \mathrm{mp}=55-57{ }^{\circ} \mathrm{C}\right) .{ }^{1} \mathrm{H} \mathrm{NMR}\left(\mathrm{CDCl}_{3}, 300 \mathrm{MHz}\right) \delta 7.98(\mathrm{~d}, J=8.4 \mathrm{~Hz}, 1 \mathrm{H}), 7.77(\mathrm{~s}, 1 \mathrm{H}), 7.67(\mathrm{~d}, J=$ $8.1 \mathrm{~Hz}, 1 \mathrm{H}), 7.59(\mathrm{dt}, J=1.4$ and $8.4 \mathrm{~Hz}, 1 \mathrm{H}), 7.41(\mathrm{t}, J=8.1 \mathrm{~Hz}, 1 \mathrm{H}), 3.12(\mathrm{t}, J=6.5 \mathrm{~Hz}, 2 \mathrm{H}), 2.94(\mathrm{t}, J$ $=6.5 \mathrm{~Hz}, 2 \mathrm{H}, 2 \mathrm{H}), 1.96(\mathrm{~m}, 2 \mathrm{H}), 1.88(\mathrm{~m}, 2 \mathrm{H}) ;{ }^{13} \mathrm{C} \mathrm{NMR}\left(\mathrm{CDCl}_{3}, 75 \mathrm{MHz}\right) \delta 159.3(\mathrm{C}), 146.6(\mathrm{C})$, $135.2(\mathrm{CH}), 131.1(\mathrm{C}), 128.6(\mathrm{CH}), 128.3(\mathrm{CH}), 127.3(\mathrm{C}), 127.0(\mathrm{CH}), 125.7(\mathrm{CH}), 33.6\left(\mathrm{CH}_{2}\right), 29.3$ $\left(\mathrm{CH}_{2}\right), 23.3\left(\mathrm{CH}_{2}\right), 23.0\left(\mathrm{CH}_{2}\right)$; IR $v_{\max }(\mathrm{KBr}) 2935,2858,1623,1491,1437,1414,1243,750 \mathrm{~cm}^{-1} ; \mathrm{MS}$, m/z (EI, 70eV) $183\left(\mathrm{M}^{+\bullet}, 100 \%\right), 182$ (79), 168 (41), 154 (37), 115 (19), 77 (14); HRMS, Found: $\mathrm{M}^{+\bullet}$, 183.1045. $\mathrm{C}_{13} \mathrm{H}_{13} \mathrm{~N}$ requires $\mathrm{M}^{+\bullet}, 183.1048$.

(E)-2-(2'-Nitrobenzylidene)cycloheptanone (24). Cross-coupling of 1-bromo-2-nitrobenzene (1, $\mathrm{R}=\mathrm{H})$ with $(E)-2$-(bromomethylene)cycloheptanone $(\mathbf{2 3})^{6}$ in the same manner as described above for the preparation of compound $\mathbf{2 1}$ gave a yellow oil on work-up. Subjection of this material to flash chromatography $(100: 3 \rightarrow 100: 6 \mathrm{v} / \mathrm{v}$ hexane-ethyl acetate gradient elution) and concentration of the appropriate fractions $\left(R_{\mathrm{f}}=0.4\right.$ in $55: 25: 10 \mathrm{v} / \mathrm{v} / \mathrm{v}$ hexane- $\mathrm{CH}_{2} \mathrm{Cl}_{2}$-ethyl acetate $)$ afforded compound $\mathbf{2 4}^{8}$ $(73 \%)$ as a yellow crystalline solid, $\mathrm{mp}=105-107^{\circ} \mathrm{C}\left(\right.$ lit. $\left.^{8} \mathrm{mp}=95-97{ }^{\circ} \mathrm{C}\right) .{ }^{1} \mathrm{H} \mathrm{NMR}\left(\mathrm{CDCl}_{3}, 300 \mathrm{MHz}\right)$ $\delta 8.12(\mathrm{dd}, J=1.2$ and $8.2 \mathrm{~Hz}, 1 \mathrm{H}), 7.71(\mathrm{~s}, 1 \mathrm{H}), 7.63(\mathrm{dt}, J=1.2$ and $7.7 \mathrm{~Hz}, 1 \mathrm{H}), 7.49(\mathrm{dt}, J=1.2$ and $8.2 \mathrm{~Hz}, 1 \mathrm{H}), 7.27(\mathrm{~d}, J=7.7 \mathrm{~Hz}, 1 \mathrm{H}), 2.71(\mathrm{~m}, 2 \mathrm{H}), 2.40(\mathrm{~m}, 2 \mathrm{H}), 1.76(\mathrm{~m}, 4 \mathrm{H}), 1.62(\mathrm{~m}, 2 \mathrm{H}) ;{ }^{13} \mathrm{C} \mathrm{NMR}$ $\left(\mathrm{CDCl}_{3}, 75 \mathrm{MHz}\right) \delta 203.6(\mathrm{C}), 148.2(\mathrm{C}), 141.6(\mathrm{C}), 133.4(\mathrm{CH}), 133.0(\mathrm{CH}), 132.8(\mathrm{C}), 131.1(\mathrm{CH})$, $128.9(\mathrm{CH}), 125.0(\mathrm{CH}), 43.5\left(\mathrm{CH}_{2}\right), 31.3\left(\mathrm{CH}_{2}\right), 29.9\left(\mathrm{CH}_{2}\right), 28.1\left(\mathrm{CH}_{2}\right), 25.5\left(\mathrm{CH}_{2}\right)$; IR $v_{\max }(\mathrm{KBr})$ 2933, 2917, 2858, 1682, 1614, 1599, 1523, 1349, 1173, $752 \mathrm{~cm}^{-1}$; MS, m/z (EI, 70eV) $245\left(\mathrm{M}^{+\bullet},<1 \%\right)$, 213 (6), 199 (27), 120 (96), 119 (100), 92 (57), 77 (30) HRMS, Found: $\mathrm{M}^{+\bullet}, 245.1050 . \mathrm{C}_{14} \mathrm{H}_{15} \mathrm{NO}_{3}$ requires: $\mathrm{M}^{+\bullet}, 245.1052$.

7,8,9,10-Tetrahydro-6H-cyclohepta[b]quinoline (25). A magnetically stirred mixture of compound 24 (310 $\mathrm{mg}, 1.26 \mathrm{mmol})$ and 10\% $\mathrm{Pd}$ on carbon $(78 \mathrm{mg})$ in methanol $(2 \mathrm{~mL})$ was exposed to dihydrogen (1 atm.). After stirring at $18{ }^{\circ} \mathrm{C}$ for $1 \mathrm{~h}$, the reaction mixture was treated with triethylamine $(1 \mathrm{~mL})$ and after a further $5 \mathrm{~h}$ filtered through a short pad of Celite ${ }^{\mathrm{TM}}$ and the filtrate concentrated under reduced pressure. The ensuing light-yellow was subjected to flash chromatography $(100: 5 \rightarrow 100: 20 \mathrm{v} / \mathrm{v}$ hexaneethyl acetate gradient elution) thus affording, after concentration of the appropriate fractions $\left(R_{\mathrm{f}}=0.5\right.$ in 55:25:20 v/v/v hexane- $\mathrm{CH}_{2} \mathrm{Cl}_{2}$-ethyl acetate), compound $\mathbf{2 5}^{9}$ (152 $\mathrm{mg}, 61 \%$ ) as a white crystalline solid, 
$\mathrm{mp}=90-91.5^{\circ} \mathrm{C}\left(\right.$ lit. $\left.^{9} \mathrm{mp}=91-92{ }^{\circ} \mathrm{C}\right) .{ }^{1} \mathrm{H} \mathrm{NMR}\left(\mathrm{CDCl}_{3}, 300 \mathrm{MHz}\right) \delta 8.00(\mathrm{~d}, J=8.4 \mathrm{~Hz}, 1 \mathrm{H}), 7.79$ (s, $1 \mathrm{H}), 7.70(\mathrm{dd}, J=1.3$ and $8.2 \mathrm{~Hz}, 1 \mathrm{H}), 7.61(\mathrm{~m}, 1 \mathrm{H}), 7.44(\mathrm{~m}, 1 \mathrm{H}), 3.21(\mathrm{~m}, 2 \mathrm{H}), 2.93(\mathrm{~m}, 2 \mathrm{H}), 1.88(\mathrm{~m}$, 2H), $1.75(\mathrm{~m}, 4 \mathrm{H}) ;{ }^{13} \mathrm{C} \mathrm{NMR}\left(\mathrm{CDCl}_{3}, 75 \mathrm{MHz}\right) \delta 164.8(\mathrm{C}), 146.2(\mathrm{C}), 136.6(\mathrm{C}), 134.8(\mathrm{CH}), 128.6$ $(\mathrm{CH}), 128.5(\mathrm{CH}), 127.5(\mathrm{C}), 126.9(\mathrm{CH}), 125.9(\mathrm{CH}), 40.1\left(\mathrm{CH}_{2}\right), 35.6\left(\mathrm{CH}_{2}\right), 32.3\left(\mathrm{CH}_{2}\right), 29.0\left(\mathrm{CH}_{2}\right)$, $27.1\left(\mathrm{CH}_{2}\right)$; IR $v_{\max }(\mathrm{KBr}) 2924,2850,1491,1448,1420,1340,750 \mathrm{~cm}^{-1} ; \mathrm{MS}, \mathrm{m} / z$ (EI, 70eV) $197\left(\mathrm{M}^{+\bullet}\right.$, 100\%), 196 (56), 182 (41), 168 (59), 115 (17); HRMS, Found: $\mathrm{M}^{+\bullet}, 197.1202 . \mathrm{C}_{14} \mathrm{H}_{15} \mathrm{~N}$ requires: $\mathrm{M}^{+\bullet}$, 197.1205.

2-(4'-Methoxy-2'-nitrobenzylidene)cyclohexanone (26). Cross-coupling of 1-bromo-4-methoxy-2nitrobenzene $(\mathbf{1}, \mathrm{R}=\mathrm{OMe})$ with $(E)$-2-(bromomethylene)cyclohexanone $(\mathbf{2 0})^{6}$ in the same manner as described above for the preparation of compound $\mathbf{2 1}$ gave a yellow oil on work-up. Subjection of this material to flash chromatography $(100: 3 \rightarrow 100: 5 \mathrm{v} / \mathrm{v}$ hexane-ethyl acetate gradient elution) and concentration of the appropriate fractions $\left(R_{\mathrm{f}}=0.3\right.$ in $55: 25: 10 \mathrm{v} / \mathrm{v} / \mathrm{v}$ hexane- $\mathrm{CH}_{2} \mathrm{Cl}_{2}$-ethyl acetate $)$ afforded compound $26(75 \%)$ as a light-yellow solid, $\mathrm{mp}=90-92{ }^{\circ} \mathrm{C} .{ }^{1} \mathrm{H}$ NMR $\left(\mathrm{CDCl}_{3}, 300 \mathrm{MHz}\right) \delta$ $7.61(\mathrm{~d}, J=2.6 \mathrm{~Hz}, 1 \mathrm{H}), 7.52(\mathrm{~m}, 1 \mathrm{H}), 7.24(\mathrm{~d}, J=9.6 \mathrm{~Hz}, 1 \mathrm{H}), 7.15(\mathrm{dd}, J=2.6$ and $9.6 \mathrm{~Hz}, 1 \mathrm{H}), 3.89$ (s, 3H), 2.55 (m, 4H), $1.92(\mathrm{~m}, 2 \mathrm{H}), 1.73(\mathrm{~m}, 2 \mathrm{H}) ;{ }^{13} \mathrm{C} \mathrm{NMR}\left(\mathrm{CDCl}_{3}, 75 \mathrm{MHz}\right) \delta 201.6(\mathrm{C}), 159.8(\mathrm{C}), 149.3$ (C), $138.3(\mathrm{C}), 132.4(\mathrm{CH}), 131.2(\mathrm{CH}), 123.8(\mathrm{C}), 119.7(\mathrm{CH}), 109.6(\mathrm{CH}), 56.1\left(\mathrm{CH}_{3}\right), 40.7\left(\mathrm{CH}_{2}\right)$, $28.9\left(\mathrm{CH}_{2}\right), 24.1\left(\mathrm{CH}_{2}\right), 23.9\left(\mathrm{CH}_{2}\right)$; IR $v_{\max }(\mathrm{KBr}) 2942,2867,1686,1619,1529,1345,1250,1141,1033$ $\mathrm{cm}^{-1}$; MS, m/z (EI, 70eV) $261\left(\mathrm{M}^{+\bullet},<1 \%\right), 229$ (7), 215 (37), 150 (59), 149 (100), 122 (22), 77 (14), 55 (21); HRMS, Found: $\mathrm{M}^{+\bullet}, 261.1010 . \mathrm{C}_{14} \mathrm{H}_{15} \mathrm{NO}_{4}$ requires: $\mathrm{M}^{+\bullet}, 261.1001$.

1,2,3,4-Tetrahydro-6-methoxyacridine (27). Reductive cyclization of compound 26 in the same manner as described above for the preparation of compound 25 afforded a light-solid on work-up. Subjection of this material to flash chromatography $(100: 30 \mathrm{v} / \mathrm{v}$ hexane-ethyl acetate elution) and concentration of the appropriate fractions $\left(R_{\mathrm{f}}=0.5\right.$ in $40: 25: 55 \mathrm{v} / \mathrm{v} / \mathrm{v}$ ethyl acetate- $\left.\mathrm{CH}_{2} \mathrm{Cl}_{2}-\mathrm{hexane}\right)$ afforded compound $27^{10}(51 \%)$ as a light-yellow crystalline solid, $\mathrm{mp}=51-52{ }^{\circ} \mathrm{C}$ (lit. ${ }^{10} \mathrm{mp}=58-59{ }^{\circ} \mathrm{C}$ ). ${ }^{1} \mathrm{H} \mathrm{NMR}\left(\mathrm{CDCl}_{3}, 300 \mathrm{MHz}\right) \delta 7.70(\mathrm{~s}, 1 \mathrm{H}), 7.55(\mathrm{~d}, J=8.9 \mathrm{~Hz}, 1 \mathrm{H}), 7.31(\mathrm{~d}, J=2.5 \mathrm{~Hz}, 1 \mathrm{H}), 7.08(\mathrm{dd}, J$ $=2.5$ and $8.9 \mathrm{~Hz}, 1 \mathrm{H}), 3.91(\mathrm{~s}, 3 \mathrm{H}), 3.08(\mathrm{t}, J=6.5 \mathrm{~Hz}, 2 \mathrm{H}), 2.91(\mathrm{t}, J=6.3 \mathrm{~Hz}, 2 \mathrm{H}), 1.96(\mathrm{~m}, 2 \mathrm{H}), 1.86$ $(\mathrm{m}, 2 \mathrm{H}) ;{ }^{13} \mathrm{C} \mathrm{NMR}\left(\mathrm{CDCl}_{3}, 75 \mathrm{MHz}\right) \delta 160.2(\mathrm{C}), 159.3(\mathrm{C}), 148.1(\mathrm{C}), 135.1(\mathrm{CH}), 128.7(\mathrm{C}), 128.0$ $(\mathrm{CH}), 122.5(\mathrm{C}), 118.9(\mathrm{CH}), 106.3(\mathrm{CH}), 55.5\left(\mathrm{CH}_{3}\right), 33.5\left(\mathrm{CH}_{2}\right), 29.8\left(\mathrm{CH}_{2}\right), 23.3\left(\mathrm{CH}_{2}\right), 23.1\left(\mathrm{CH}_{2}\right)$; IR $v_{\max }(\mathrm{KBr}) 2933,2858,1626,1498,1454,1239,1031,732 \mathrm{~cm}^{-1} ; \mathrm{MS}, \mathrm{m} / z$ (EI, 70eV) $213\left(\mathrm{M}^{+\bullet}, 100 \%\right)$, 212 (44), 198 (39), 185 (24), 170 (18), 115 (12); HRMS, Found: $\mathrm{M}^{+\bullet}, 213.1151 . \mathrm{C}_{14} \mathrm{H}_{15} \mathrm{NO}$ requires: $\mathrm{M}^{+\bullet}$, 213.1154 .

1-(2'-Nitrophenyl)-3,4-dihydronaphthalene-2-carbaldehyde (29). Cross-coupling of 1-bromo-2nitrobenzene $(\mathbf{1}, \mathrm{R}=\mathrm{H})$ with aldehyde $28^{11}$ in the same manner as described above for the preparation of compound 7 gave an oily solid on work-up. Subjection of this material to flash chromatography $(3: 1 \mathrm{v} / \mathrm{v}$ 
hexane-ethyl acetate elution) and concentration of the appropriate fractions $\left(R_{\mathrm{f}}=0.4\right)$ afforded compound $29(93 \%)$ as a yellow powder, $\mathrm{mp}=118{ }^{\circ} \mathrm{C} .{ }^{1} \mathrm{H}$ NMR $\left(\mathrm{CDCl}_{3}, 300 \mathrm{MHz}\right) \delta 9.49(\mathrm{~s}, 1 \mathrm{H}), 8.26(\mathrm{ddd}, J=$ 0.6, 1.2 and $8.1 \mathrm{~Hz}, 1 \mathrm{H}), 7.77(\mathrm{dt}, J=1.5$ and $7.5 \mathrm{~Hz}, 1 \mathrm{H}), 7.68(\mathrm{~m}, 1 \mathrm{H}), 7.44(\mathrm{dd}, J=1.5 \mathrm{and} 7.5 \mathrm{~Hz}$, 1H), 7.29 (complex m, 1H), 7.28 (d, $J=0.9 \mathrm{~Hz}, 1 \mathrm{H}), 7.08$ (m, 1H), 6.59 (d, $J=7.5 \mathrm{~Hz}, 1 \mathrm{H}), 3.10-2.83$ (complex m, 3H), 2.61-2.49 (complex m, 1H); ${ }^{13} \mathrm{C} \mathrm{NMR}\left(\mathrm{CDCl}_{3}, 75 \mathrm{MHz}\right) \delta 191.3,151.1,148.7,138.4$, $134.2,133.8,133.4,133.1,131.2,130.5,129.9,128.1,126.8,126.3,125.2,27.2,20.0 ; \operatorname{IR} v_{\max }(\mathrm{NaCl})$ 2848, 1665, 1525, 1345, 855, 777, $721 \mathrm{~cm}^{-1}$; MS, m/z (EI, 70eV) $279\left(\mathrm{M}^{+\bullet}, 9 \%\right), 250$ (76), 231 (36), 217 (25), 206 (100), 202 (89), 189 (22), 180 (36), 165 (22), 101 (24); HRMS, Found: M+, 279.0895. $\mathrm{C}_{17} \mathrm{H}_{13} \mathrm{NO}_{3}$ requires $\mathrm{M}^{+\bullet}, 279.0895$.

7,8-Dihydrobenzo $[k]$ phenanthridine (30). Reductive cyclization of compound 29 in the same manner as described above for the preparation of compound $\mathbf{1 1}$ afforded a light-yellow oil on work-up. Subjection of this material to flash chromatography (47:47:6 v/v/v hexane-ethyl acetate-triethylamine elution) and concentration of the appropriate fractions $\left(R_{\mathrm{f}}=0.4\right)$ afforded compound $\mathbf{3 0}^{11}(87 \%)$ as a light-yellow oil. ${ }^{1} \mathrm{H} \mathrm{NMR}\left(\mathrm{CDCl}_{3}, 300 \mathrm{MHz}\right) \delta 8.83(\mathrm{~s}, 1 \mathrm{H}), 8.51(\mathrm{~d}, J=8.4 \mathrm{~Hz}, 1 \mathrm{H}), 8.15(\mathrm{dd}, J=0.9$ and $8.4 \mathrm{~Hz}, 1 \mathrm{H})$, 8.01-7.98 (complex m, 1H), 7.69 (two overlapping dd, $J=1.2$ and $6.9 \mathrm{~Hz}, 1 \mathrm{H}$ ), 7.57 (two overlapping dd, $J=1.4$ and $6.9 \mathrm{~Hz}, 1 \mathrm{H}$ ), 7.45-7.38 (complex m, 3H), 2.96-2.85 (complex m, 4H); IR $v_{\max }\left(\mathrm{CH}_{2} \mathrm{Cl}_{2}\right)$ 1651, 1607, 1569, 1558, 1505, $1487 \mathrm{~cm}^{-1}$; MS, m/z (EI, 70eV) $231\left(\mathrm{M}^{+\bullet}, 100 \%\right), 230$ (78), 202 (37), 101 (24).

1-(4'-Methoxy-2'-nitrophenyl)-3,4-dihydronaphthalene-2-carbaldehyde (31). Cross-coupling of 1-bromo-4-methoxy-2-nitrobenzene $(\mathbf{1}, \mathrm{R}=\mathrm{OMe})$ with aldehyde $\mathbf{2 8}^{11}$ in the same manner as described above for the preparation of compound 7 gave a yellow crystalline solid on work-up. Subjection of this material to flash chromatography $(4: 1 \mathrm{v} / \mathrm{v}$ hexane-ethyl acetate elution) and concentration of the appropriate fractions $\left(R_{\mathrm{f}}=0.7\right)$ afforded compound $\mathbf{3 1}(89 \%)$ as a yellow crystalline solid, $\mathrm{mp}=204{ }^{\circ} \mathrm{C}$. ${ }^{1} \mathrm{H}$ NMR $\left(\mathrm{CDCl}_{3}, 300 \mathrm{MHz}\right) \delta 9.52(\mathrm{~s}, 1 \mathrm{H}), 7.75(\mathrm{~d}, J=2.1 \mathrm{~Hz}, 1 \mathrm{H}), 7.33-7.24$ (complex m, 4H), 7.10-7.05 (complex m, 1H), 6.63 (d, $J=7.5 \mathrm{~Hz}, 1 \mathrm{H}), 3.96(\mathrm{~s}, 3 \mathrm{H}), 3.07-2.81$ (complex m, 3H), 2.58-2.47 (complex m, 1H); ${ }^{13} \mathrm{C} \mathrm{NMR}\left(\mathrm{CDCl}_{3}, 75 \mathrm{MHz}\right) \delta 191.6,160.3,151.1,149.4,138.5,134.4$, $134.2,133.9,130.4,128.0,126.7,126.2,122.9,119.8,109.9,56.0,27.2,20.1 ; \mathrm{IR} v_{\max }(\mathrm{NaCl}) 2840,1663$, 1531, 1347, 1300, 1031, 775, $736 \mathrm{~cm}^{-1}$; MS, m/z (EI, 70eV) 309 (M+*, 14\%), 280 (60), 263 (42), 236 (100), 189 (61), 165 (30), 152 (17), 115 (16), 63 (11); HRMS, Found: $\mathrm{M}^{+\bullet}, 309.0999 . \mathrm{C}_{18} \mathrm{H}_{15} \mathrm{NO}_{4}$ requires $\mathbf{M}^{+\bullet}, 309.1001$.

3-Methoxy-7,8-dihydrobenzo $[k]$ phenanthridine (32). Reductive cyclization of compound 31 in the same manner as described above for the preparation of compound $\mathbf{1 1}$ afforded a light-yellow oil on workup. Subjection of this material to flash chromatography $(47: 47: 6 \mathrm{v} / \mathrm{v} / \mathrm{v}$ hexane-ethyl acetate-triethylamine elution) and concentration of the appropriate fractions $\left(R_{\mathrm{f}}=0.2\right)$ afforded compound $32(91 \%)$ as a yellow oil. ${ }^{1} \mathrm{H}$ NMR $\left(\mathrm{CDCl}_{3}, 300 \mathrm{MHz}\right) \delta 8.34(\mathrm{~d}, J=9.6 \mathrm{~Hz}, 1 \mathrm{H}), 8.33$ (s, 1H), 8.12 (d, $\left.J=2.7 \mathrm{~Hz}, 1 \mathrm{H}\right), 7.79$ 
$(\mathrm{d}, J=6.6 \mathrm{~Hz}, 1 \mathrm{H}), 7.42-7.34$ (complex m, 3H), $7.27(\mathrm{dd}, J=2.7$ and $9.6 \mathrm{~Hz}, 1 \mathrm{H}), 4.00(\mathrm{~s}, 3 \mathrm{H})$, 2.84-2.69 (complex m, 4H); ${ }^{13} \mathrm{C} \mathrm{NMR}\left(\mathrm{CDCl}_{3}, 75 \mathrm{MHz}\right) \delta 160.9,142.4,139.6,135.4,132.0,131.2$, $129.3,128.7,128.6,128.4,127.5,126.7,121.8,121.6,98.4,55.9,28.7,26.5 ;$ IR $v_{\max }(\mathrm{NaCl}) 2927,1645$, $1624,1481,1384,1275,1225,1199,1164,1026 \mathrm{~cm}^{-1}$; MS, $m / z(\mathrm{EI}, 70 \mathrm{eV}) 277\left([\mathrm{M}+\mathrm{O}]^{+\bullet}, 100 \%\right), 261$ (M+•, 99\%), 247 (14), 230 (20), 217 (28), 189 (25); HRMS, Found: $\mathrm{M}^{+\bullet}, 261.1156 . \mathrm{C}_{18} \mathrm{H}_{15} \mathrm{NO}$ requires $\mathrm{M}^{+\bullet}, 261.1154$.

4,5-Methylenedioxy-2'-nitro-2-biphenylcarbaldehyde (34). Cross-coupling of 1-bromo-2nitrobenzene $(\mathbf{1}, \mathrm{R}=\mathrm{H})$ with aldehyde $\mathbf{3 3}$ in the same manner as described above for the preparation of compound 7 gave a yellow solid on work-up. Subjection of this material to flash chromatography $(4: 1 \mathrm{v} / \mathrm{v}$ hexane-ethyl acetate elution) and concentration of the appropriate fractions $\left(R_{\mathrm{f}}=0.45 \mathrm{in} 7: 3 \mathrm{v} / \mathrm{v}\right.$ hexaneethyl acetate) afforded compound $\mathbf{3 4}^{12}(57 \%)$ as a yellow solid, $\mathrm{mp}=135-138{ }^{\circ} \mathrm{C}\left(\right.$ lit. $^{12} \mathrm{mp}=227-228$ $\left.{ }^{\circ} \mathrm{C}\right) .{ }^{1} \mathrm{H}$ NMR $\left(\mathrm{CDCl}_{3}, 300 \mathrm{MHz}\right) \delta 9.57(\mathrm{~s}, 1 \mathrm{H}), 8.07(\mathrm{dd}, J=1.8$ and $7.5 \mathrm{~Hz}, 1 \mathrm{H}), 7.67(\mathrm{dt}, J=1.5$ and $7.5 \mathrm{~Hz}, 1 \mathrm{H}), 7.60(\mathrm{dt}, J=1.8$ and $7.5 \mathrm{~Hz}, 1 \mathrm{H}), 7.45(\mathrm{~s}, 1 \mathrm{H}), 7.37$ (dd, $J=1.5$ and $7.5 \mathrm{~Hz}, 1 \mathrm{H}), 6.67$ (s, $1 \mathrm{H}), 6.12(\mathrm{~m}, 2 \mathrm{H}) ;{ }^{13} \mathrm{C} \mathrm{NMR}\left(\mathrm{CDCl}_{3}, 75 \mathrm{MHz}\right) \delta 188.8,152.1,149.1,148.4,137.7,132.8,132.6,129.4$, $128.8,124.4,109.5,107.2,102.4$ (one signal obscured or overlapping); IR $v_{\max }$ (KBr) 1678, 1609, 1523, 1476, 1407, 1360, 1349, 1270, 1255, 1230, $1035 \mathrm{~cm}^{-1}$; MS, m/z (EI, 70eV) $271\left(\mathrm{M}^{+\bullet}, 30 \%\right), 242$ (20), 225 (100), 198 (24), 139 (65); HRMS, Found: $\mathrm{M}^{+\bullet}, 271.0471 \mathrm{C}_{14} \mathrm{H}_{9} \mathrm{NO}_{5}$ requires $\mathrm{M}^{+\bullet}, 271.0481$.

Trispheridine $\{[1,3]$ Dioxolo[4,5-j]phenanthridine (35). Reductive cyclization of compound 34 in the same manner as described above for the preparation of compound 11 afforded compound $\mathbf{3 5}^{13}(68 \%)$ as a pale-yellow solid, $\mathrm{mp}=111-125^{\circ} \mathrm{C}$, [lit. ${ }^{13} \mathrm{mp}=144.5-145^{\circ} \mathrm{C}$ (sublimation from $119{ }^{\circ} \mathrm{C}$ onwards) $]$. ${ }^{1} \mathrm{H}$ NMR $\left(\mathrm{CDCl}_{3}, 300 \mathrm{MHz}\right) \delta 9.09(\mathrm{~s}, 1 \mathrm{H}), 8.38(\mathrm{~d}, J=7.2 \mathrm{~Hz}, 1 \mathrm{H}), 8.14(\mathrm{~d}, J=8.7 \mathrm{~Hz}, 1 \mathrm{H}), 7.91$ (s, $1 \mathrm{H}), 7.69(\mathrm{t}, J=8.7 \mathrm{~Hz}, 1 \mathrm{H}), 7.63(\mathrm{~d}, J=7.2 \mathrm{~Hz}, 1 \mathrm{H}), 7.34(\mathrm{~s}, 1 \mathrm{H}), 6.17(\mathrm{~s}, 2 \mathrm{H}) ;{ }^{13} \mathrm{C} \mathrm{NMR}\left(\mathrm{CDCl}_{3}, 75\right.$ MHz) $\delta 151.7,151.5,148.2,144.0,130.2$, 130.0, 128.0, 126.7, 124.2, 123.0, 122.0, 105.5, 101.9, 99.9; IR $v_{\max }(\mathrm{KBr}) 2916,1620,1583,1499,1484,1464,1393,1256,1226,1197,1038,942,830,760 \mathrm{~cm}^{-1}$; MS, m/z (EI, 70eV) 223 (M+•, 100\%), 164 (12), 138 (20), 111 (10), 86 (42), 84 (57); HRMS, Found: M $^{+\bullet}$ 223.0631 $\mathrm{C}_{14} \mathrm{H}_{9} \mathrm{NO}_{2}$ requires $\mathrm{M}^{+\bullet}, 223.0633$.

7-Methoxy-8-(2'-nitrophenyl)-3,4,-dihydro-2H-naphthalen-1-one (37). A magnetically stirred mixture of 1-bromo-2-nitrobenzene $(1, \mathrm{R}=\mathrm{H})(300 \mathrm{mg}, 1.5 \mathrm{mmol}), \mathrm{Pd}_{2}(\mathrm{dba})_{3}(25 \mathrm{mg}, 5 \mathrm{~mol} \%)$ and copper powder $(160 \mathrm{mg}, 2.5 \mathrm{mmol})$ in DMSO $(3 \mathrm{~mL})$ was heated to $80{ }^{\circ} \mathrm{C}$ whilst being maintained under a nitrogen atmosphere then a solution of tetralone $36^{14}(125 \mathrm{mg}, 0.5 \mathrm{mmol})$ in DMSO $(1 \mathrm{~mL})$ was added via syringe pump over a period of $1 \mathrm{~h}$. After addition was complete the reaction mixture was maintained at $80{ }^{\circ} \mathrm{C}$ for $4 \mathrm{~h}$. Work-up of the cooled reaction mixture in the same manner as described for the preparation of compound 7 gave a yellow powder. Subjection of this material to flash chromatography (3:2 v/v hexane-ethyl acetate elution) afforded two major fractions, A and B. 
Concentration of fraction $\mathrm{A}\left(R_{\mathrm{f}}=0.6\right)$ afforded a mixture of the desired cross-coupling product and 2,2'-dinitrobiphenyl [product from reductive dimerization of compound $(\mathbf{1} \mathrm{R}=\mathrm{H})$ ].

Concentration of fraction $\mathrm{B} \quad\left(R_{\mathrm{f}}=0.3\right)$ afforded 2,2'-dimethoxy-6,6'7',7'-5H,5'H[1,1]binaphthalenyl-8,8'-dione (product from reductive dimerization of compound 36) (34\%) as a white crystalline solid, $\mathrm{mp}=178-180{ }^{\circ} \mathrm{C} .{ }^{1} \mathrm{H} \mathrm{NMR}\left(\mathrm{CDCl}_{3}, 300 \mathrm{MHz}\right) \delta 7.21(\mathrm{~d}, J=8.4 \mathrm{~Hz}, 2 \mathrm{H}), 7.07$ (d, $J=8.4 \mathrm{~Hz}, 2 \mathrm{H}), 3.63(\mathrm{~s}, 6 \mathrm{H}), 2.96$ (complex m, 4H), 2.47 (t, $J=6.6 \mathrm{~Hz}, 4 \mathrm{H}$ ), 2.16-1.98 (complex m, $4 \mathrm{H}) ;{ }^{13} \mathrm{C} \mathrm{NMR}\left(\mathrm{CDCl}_{3}, 75 \mathrm{MHz}\right) \delta 198.6,155.0,136.9,131.4,128.8,128.1,115.8,56.4,40.0,30.0,23.2$; IR $v_{\max }(\mathrm{NaCl}) 2923,2857,1681,1483,1268,1219,1189,1027,988,921,824 \mathrm{~cm}^{-1} ; \mathrm{MS}, \mathrm{m} / z$ (EI, 70eV)

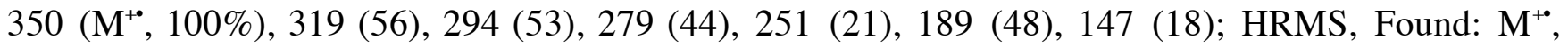
350.1520. $\mathrm{C}_{22} \mathrm{H}_{22} \mathrm{O}_{4}$ requires $\mathrm{M}^{+\bullet}, 350.1518$.

Subjection of fraction A, obtained as described above, to flash chromatography (4:1 v/v dichloromethane-pentane elution) and concentration of the appropriate fractions $\left(R_{\mathrm{f}}=0.6\right)$ afforded compound $37(53 \%)$ as a yellow solid, $\mathrm{mp}=204{ }^{\circ} \mathrm{C} .{ }^{1} \mathrm{H} \mathrm{NMR}\left(\mathrm{CDCl}_{3}, 300 \mathrm{MHz}\right) \delta 8.16(\mathrm{dd}, J=1.2$ and $8.4 \mathrm{~Hz}, 1 \mathrm{H}), 7.59(\mathrm{dt}, J=1.5$ and $7.5 \mathrm{~Hz}, 1 \mathrm{H}), 7.47(\mathrm{dt}, J=1.5$ and $7.5 \mathrm{~Hz}, 1 \mathrm{H}), 7.30(\mathrm{~d}, J=8.4 \mathrm{~Hz}, 1 \mathrm{H})$, $7.12(\mathrm{dd}, J=1.5$ and $7.5 \mathrm{~Hz}, 1 \mathrm{H}), 7.10(\mathrm{~d}, J=8.4 \mathrm{~Hz}, 1 \mathrm{H}), 3.65(\mathrm{~s}, 3 \mathrm{H}), 2.98(\mathrm{t}, J=6.3 \mathrm{~Hz}, 2 \mathrm{H}), 2.53(\mathrm{t}, J$

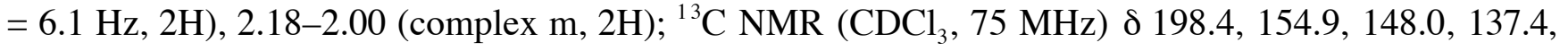
$134.8,132.8,131.2,130.8,129.6,128.0,127.3,124.2,115.8,56.1,40.0,29.8,23.1 ;$ IR $v_{\max }(\mathrm{NaCl}) 2937$, 1678, 1520, 1347, 1270, 1028, 936, 854, 788, $754 \mathrm{~cm}^{-1}$; MS, m/z (EI, 70eV) $297\left(\mathrm{M}^{+\bullet}, 1 \%\right), 251$ (100), 236 (12), 198 (11), 149 (7); HRMS, Found: $\mathrm{M}^{+\bullet}, 297.1012 . \mathrm{C}_{17} \mathrm{H}_{15} \mathrm{NO}_{4}$ requires $\mathrm{M}^{+\bullet}, 297.1001$.

5,6-Dihydro-11-Methoxy-4H-benz[ $[\boldsymbol{k l}]$ acridine (38). Reductive cyclization of compound 37 in the same manner as described above for the preparation of compound $\mathbf{1 1}$ afforded a clear colorless oil on work-up. Subjection of this material to flash chromatography $(63: 31: 6 \mathrm{v} / \mathrm{v} / \mathrm{v}$ hexane-ethyl acetatetriethylamine elution) and concentration of the appropriate fractions $\left(R_{\mathrm{f}}=0.4\right)$ afforded compound 38 (92\%) as a clear, yellow oil. ${ }^{1} \mathrm{H} \mathrm{NMR}\left(\mathrm{CDCl}_{3}, 300 \mathrm{MHz}\right) \delta 9.45(\mathrm{~d}, J=8.4 \mathrm{~Hz}, 1 \mathrm{H}), 8.17(\mathrm{~d}, J=8.4 \mathrm{~Hz}$, $1 \mathrm{H}), 7.69(\mathrm{t}, J=8.4 \mathrm{~Hz}, 1 \mathrm{H}), 7.58(\mathrm{t}, J=8.4 \mathrm{~Hz}, 1 \mathrm{H}), 7.40(\mathrm{~d}, J=8.4 \mathrm{~Hz}, 1 \mathrm{H}), 7.23(\mathrm{~d}, J=8.1 \mathrm{~Hz}, 1 \mathrm{H})$, $4.09(\mathrm{~s}, 3 \mathrm{H}), 3.36(\mathrm{t}, J=6.0 \mathrm{~Hz}, 2 \mathrm{H}), 3.12(\mathrm{t}, J=6.0 \mathrm{~Hz}, 2 \mathrm{H}), 2.22-2.14$ (complex m, $2 \mathrm{H}) ;{ }^{13} \mathrm{C}$ NMR $\left(\mathrm{CDCl}_{3}, 75 \mathrm{MHz}\right) \delta 159.7,156.3,143.9,131.1,128.7,127.9,126.7,125.8,124.8,123.7,123.0,111.7$, $55.7,35.7,30.8,23.2$ (one signal obscured or overlapping); IR $v_{\max }(\mathrm{NaCl}) 2926,2855,1574,1455,1435$, 1361, 1248, 1122, 1046, 1027, 814, $765 \mathrm{~cm}^{-1}$; MS, m/z (EI, 70eV) 249 (M+•, 100\%), 234 (45), 206 (19), 204 (22); HRMS, Found: $\mathrm{M}^{+\bullet}, 249.1148 . \mathrm{C}_{17} \mathrm{H}_{15} \mathrm{NO}$ requires $\mathrm{M}^{+\bullet}, 249.1154$.

3-(2'-Nitrophenyl)-1H-indole-2-carbaldehyde (40). Cross-coupling of 1-bromo-2-nitrobenzene (1, $\mathrm{R}=\mathrm{H}$ ) with aldehyde $39^{15}$ in the same manner as described above for the preparation of compound 7 gave a yellow powder on work-up. Subjection of this material to flash chromatography $(1: 1 \mathrm{v} / \mathrm{v}$ hexane-ethyl acetate elution) and concentration of the appropriate fractions $\left(R_{\mathrm{f}}=0.7\right)$ afforded a mixture of the desired cross-coupling product and 2,2'-dinitrobiphenyl [product from reductive dimerization of compound ( $\mathbf{1}$ 
$\mathrm{R}=\mathrm{H})$ ]. Subjection of this material to flash chromatography $(1: 1 \mathrm{v} / \mathrm{v}$ hexane-diethyl ether elution) and concentration of the appropriate fractions $\left(R_{\mathrm{f}}=0.4\right)$ then afforded compound $\mathbf{4 0}(63 \%)$ as a powder solid, $\mathrm{mp}=158{ }^{\circ} \mathrm{C} .{ }^{1} \mathrm{H} \mathrm{NMR}\left(\mathrm{CDCl}_{3}, 300 \mathrm{MHz}\right) \delta 9.72(\mathrm{~s}, 1 \mathrm{H}), 9.33($ broad s, 1H), $8.11(\mathrm{dd}, J=1.5$ and 7.5 $\mathrm{Hz}, 1 \mathrm{H}), 7.74(\mathrm{dt}, J=1.5$ and $7.5 \mathrm{~Hz}, 1 \mathrm{H}), 7.65(\mathrm{dt}, J=1.8$ and $8.1 \mathrm{~Hz}, 1 \mathrm{H}), 7.58(\mathrm{dd}, J=1.8$ and $7.5 \mathrm{~Hz}$, $1 \mathrm{H}), 7.48(\mathrm{t}, J=8.4 \mathrm{~Hz}, 1 \mathrm{H}), 7.45-7.38$ (complex m, 2H), 7.19-7.13 (complex m, 1H); ${ }^{13} \mathrm{C}$ NMR $\left(\mathrm{CDCl}_{3}, 75 \mathrm{MHz}\right) \delta 181.4,136.7,134.2,132.6,132.0,129.4,127.9,126.9,126.5,124.8(4), 124.8(0)$, 123.8, 121.9, 121.1, 112.7; IR $v_{\max }(\mathrm{NaCl}) 1652,1525,1349,1333,1231,1231,1016,867,851,785,740$, $702 \mathrm{~cm}^{-1}$; MS, m/z (EI, 70eV) $266\left(\mathrm{M}^{+\bullet}, 100 \%\right), 221$ (64), 219 (60), 210 (83), 209 (93), 190 (49), 181 (67), 165 (68), 152 (44), 139 (24), 77 (28), 63 (24); HRMS, Found: $\mathrm{M}^{+\bullet}, 266.0689 . \mathrm{C}_{15} \mathrm{H}_{10} \mathrm{~N}_{2} \mathrm{O}_{3}$ requires $\mathrm{M}^{+\bullet}, 266.0691$.

7H-Indolo[2,3-c] $]$ quinoline (41). Reductive cyclization of compound 40 in the same manner as described above for the preparation of compound $\mathbf{1 1}$ afforded a clear colorless oil on work-up. Subjection of this material to flash chromatography $(42: 42: 8 \mathrm{v} / \mathrm{v} / \mathrm{v}$ hexane-ethyl acetate-triethylamine elution) and concentration of the appropriate fractions $\left(R_{\mathrm{f}}=0.1\right)$ afforded compound $\mathbf{4 1}^{16}(66 \%)$ as a yellow powder, $\mathrm{mp}=251{ }^{\circ} \mathrm{C}\left(\right.$ lit. $\left.^{16} \mathrm{mp}=249{ }^{\circ} \mathrm{C}\right) .{ }^{1} \mathrm{H}$ NMR $\left[\left(\mathrm{CD}_{3}\right)_{2} \mathrm{CO}, 300 \mathrm{MHz}\right] \delta 11.24($ broad s, $1 \mathrm{H}), 9.31(\mathrm{~s}, 1 \mathrm{H})$, $8.81(\mathrm{dd}, J=1.2$ and $8.4 \mathrm{~Hz}, 1 \mathrm{H}), 8.68(\mathrm{~d}, J=8.1 \mathrm{~Hz}, 1 \mathrm{H}), 8.22(\mathrm{dd}, J=1.2$ and $8.1 \mathrm{~Hz}, 1 \mathrm{H}), 7.81-7.73$ (complex m, 2H), 7.65 (ddd, $J=1.5,6.9$ and 8.1 Hz, 1H), 7.59 (ddd, $J=1.2,7.2$ and 8.1 Hz, 1H), 7.42 (ddd, $J=1.2,7.2$ and $8.1 \mathrm{~Hz}, 1 \mathrm{H}) ;{ }^{13} \mathrm{C} \mathrm{NMR}\left[\left(\mathrm{CD}_{3}\right)_{2} \mathrm{CO}, 75 \mathrm{MHz}\right] \delta 143.9,140.6,139.5,133.9,131.3$, 127.8, 127.5, 126.0, 125.6, 124.1, 123.8, 122.9, 121.3, 121.0, 113.4; IR $v_{\max }$ (KBr disc) 2924, 2853, 1622, 1341, 1137, 1110, 762, $745 \mathrm{~cm}^{-1}$; MS, m/z (EI, 70eV) $218\left(\mathrm{M}^{+\bullet}, 100 \%\right), 190$ (12), 164 (6), 163 (6), 109 (7); HRMS, Found: $\mathrm{M}^{+\bullet}, 218.0837 . \mathrm{C}_{15} \mathrm{H}_{10} \mathrm{~N}_{2}$ requires $\mathrm{M}^{+\bullet}, 218.0844$.

2-(2'-Nitrophenyl)cyclohex-1-ene Carboxylic Acid Methyl Ester (43). Cross-coupling of 1-bromo2-nitrobenzene $(\mathbf{1}, \mathrm{R}=\mathrm{H})$ with bromo-ester $\mathbf{4 2}^{17}$ was carried out in the same manner as described above for the preparation of compound 7 save for the use of a reaction temperature of $90{ }^{\circ} \mathrm{C}$ and a reaction time of 6 h. The yellow oil obtained on work-up was subjected to flash chromatography $(9: 1 \mathrm{v} / \mathrm{v}$ hexane-ethyl acetate elution) and three fractions, A-C, were obtained.

Concentration of fraction A $\left(R_{\mathrm{f}}=0.4\right)$ afforded the starting ester $\mathbf{4 2}$ (8\% recovery) which was identical, in all respects, with an authentic sample.

Concentration of fraction $\mathrm{B}\left(R_{\mathrm{f}}=0.2\right)$ afforded 2,2 '-dinitrobiphenyl (18\% at $92 \%$ conversion) which was identical, in all respects, with an authentic sample.

Concentration of fraction $\mathrm{C}\left(R_{\mathrm{f}}=0.1\right)$ afforded compound 43 (39\% at $92 \%$ conversion) as a yellow oil. ${ }^{1} \mathrm{H} \mathrm{NMR}\left(\mathrm{CDCl}_{3}, 300 \mathrm{MHz}\right) \delta 8.04(\mathrm{dd}, J=1.2$ and $8.2 \mathrm{~Hz}, 1 \mathrm{H}), 7.55(\mathrm{dt}, J=1.2$ and $7.4 \mathrm{~Hz}$, $1 \mathrm{H}), 7.39(\mathrm{ddd}, J=1.5,7.4$ and $8.2 \mathrm{~Hz}, 1 \mathrm{H}), 7.10(\mathrm{dd}, J=1.5$ and $7.4 \mathrm{~Hz}, 1 \mathrm{H}), 3.38(\mathrm{~s}, 3 \mathrm{H}), 2.60-2.18$ (complex m, 4H), 1.86-1.54 (complex m, 4H); ${ }^{13} \mathrm{C} \mathrm{NMR}\left(\mathrm{CDCl}_{3}, 126 \mathrm{MHz}\right) \delta 167.6(\mathrm{CO}), 147.0$ (C), $146.9(\mathrm{C}), 139.8(\mathrm{C}), 133.2(\mathrm{CH}), 129.0(\mathrm{CH}), 127.5(\mathrm{CH}), 125.8(\mathrm{C}), 124.2(\mathrm{CH}), 51.3\left(\mathrm{CH}_{3}\right), 33.6$ 
$\left(\mathrm{CH}_{2}\right), 25.8\left(\mathrm{CH}_{2}\right), 22.2\left(\mathrm{CH}_{2}\right), 21.9\left(\mathrm{CH}_{2}\right) ; \mathrm{IR} v_{\max }(\mathrm{KBr}) 2935,1716,1524,1348,1241,1052 \mathrm{~cm}^{-1} ; \mathrm{MS}$, m/z (EI, 70eV) $261\left(\mathrm{M}^{+\bullet}, 2 \%\right), 243$ (40), 215 (78), 202 (70), 146 (100\%), 132 (93), 130 (70), 115 (64), 77 (60); HRMS, Found: $\mathrm{M}^{+\bullet}, 261.0999 . \mathrm{C}_{14} \mathrm{H}_{15} \mathrm{NO}_{4}$ requires $\mathrm{M}^{+\bullet}, 261.1001$

7,8,9,10-Tetrahydro-6(5H)-phenanthridinone (44). Reductive cyclization of compound 43 was achieved in the same manner as described above for the preparation of compound $\mathbf{1 1}$ save for the use of a $1 \mathrm{~h}$ reaction time. In this manner a clear, colorless oil was obtained on work-up. Subjection of this material to flash chromatography (95:4.25:0.75 v/v/v dichloromethane-methanol-15\% aq. ammonia elution) and concentration of the appropriate fractions $\left(R_{\mathrm{f}}=0.15\right)$ afforded an orange solid. Recrystallization (from ethyl acetate-hexane) of this material yielded compound $\mathbf{4 4}^{18}(78 \%)$ as a white crystalline masses, $\mathrm{mp}=$ 263-265 ${ }^{\circ} \mathrm{C}\left(\right.$ lit. $\left.{ }^{18} \mathrm{mp}=268-270{ }^{\circ} \mathrm{C}\right) .{ }^{1} \mathrm{H} \mathrm{NMR}\left(\mathrm{CD}_{3} \mathrm{OD}, 300 \mathrm{MHz}\right) \delta 7.76(\mathrm{dd}, J=0.9$ and $8.1 \mathrm{~Hz}, 1 \mathrm{H})$, $7.46(\mathrm{~m}, 1 \mathrm{H}), 7.33-7.22$ (complex m, 2H), $2.93(\mathrm{~m}, 2 \mathrm{H}), 2.58(\mathrm{~m}, 2 \mathrm{H}), 1.95-1.78$ (complex m, $4 \mathrm{H}) ;{ }^{13} \mathrm{C}$

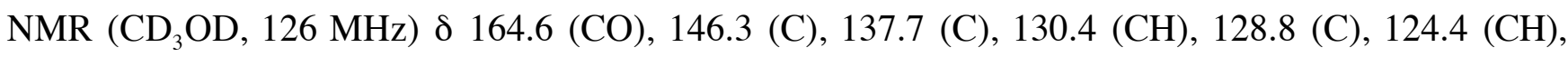
$123.7(\mathrm{CH}), 121.8(\mathrm{C}), 116.7(\mathrm{CH}), 26.6\left(\mathrm{CH}_{2}\right), 24.9\left(\mathrm{CH}_{2}\right), 23.0\left(\mathrm{CH}_{2}\right), 22.9\left(\mathrm{CH}_{2}\right)$; IR $v_{\max }(\mathrm{KBr}) 2935$, 2854, 1648, 1566, 1505, 1437, $746 \mathrm{~cm}^{-1}$; MS, m/z (EI, 70eV) $199\left(\mathrm{M}^{+\bullet}, 100 \%\right), 198$ (73), 184 (67); HRMS, Found: $\mathrm{M}^{+\bullet}, 199.0995 . \mathrm{C}_{13} \mathrm{H}_{13} \mathrm{NO}$ requires $\mathrm{M}^{+\bullet}, 199.0997$

2'Nitro-[1,1'-biphenyl]-2-carboxylic Acid Methyl Ester (46). Cross-coupling of 1-iodo-2nitrobenzene with commercially available iodo-ester 45 was carried out in the same manner as described above for the preparation of compound 7 save for the use of a reaction temperature of $90{ }^{\circ} \mathrm{C}$ and a reaction time of $2.5 \mathrm{~h}$. The yellow solid obtained on work-up was subjected to flash chromatography (9:1 v/v hexane-ethyl acetate elution) and two fractions, A and B, thereby obtained.

Concentration of fraction $\mathrm{A}\left(R_{\mathrm{f}}=0.3\right)$ afforded 2,2'-dinitrobiphenyl $(78 \%)$ which was identical, in all respects, with an authentic sample.

Concentration of fraction B $\left(R_{\mathrm{f}}=0.2\right)$ afforded a $c a$. 1:1 mixture of compound 46 and [1,1'biphenyl]-2,2'-dicarboxylic acid dimethyl ester (product from reductive dimerization of compound 45) as a yellow oil. This mixture was immediately subject to a reductive cyclization reaction as detailed below.

6(5H)-phenanthridinone (47). A $c a$. 1:1 mixture of compound 46 and [1,1'-biphenyl]-2,2'dicarboxylic acid dimethyl ester, obtained as detailed immediately above, was subjected to reductive cyclization in the same manner as described earlier for the preparation of compound $\mathbf{1 1}$ save for use of a reaction time of $0.75 \mathrm{~h}$. In this way a clear colorless oil was obtained on work-up. Subjection of this material to flash chromatography (70:27:3 v/v hexane-ethyl acetate-triethylamine elution) afforded two fractions, $\mathrm{A}$ and $\mathrm{B}$.

Concentration of fraction A $\left(R_{\mathrm{f}}=0.5\right.$ in $7: 3 \mathrm{v} / \mathrm{v}$ hexane-ethyl acetate $)$ afforded pure [1,1'biphenyl]-2,2' -dicarboxylic acid dimethyl ester $^{19}$ (40\%) as light-pink crystalline masses, $\mathrm{mp}=72-73$ 
${ }^{\circ} \mathrm{C}$ (lit. $\left.{ }^{19} \mathrm{mp}=73-74{ }^{\circ} \mathrm{C}\right) .{ }^{1} \mathrm{H}$ NMR $\left(\mathrm{CDCl}_{3}, 300 \mathrm{MHz}\right) \delta 7.99(\mathrm{dd}, J=1.4$ and $7.7 \mathrm{~Hz}, 2 \mathrm{H}), 7.52(\mathrm{dt}, J=$ 1.4 and $7.5 \mathrm{~Hz}, 2 \mathrm{H}), 7.41(\mathrm{dt}, J=1.4$ and $7.7 \mathrm{~Hz}, 2 \mathrm{H}), 7.18(\mathrm{dd}, J=1.4$ and $7.5 \mathrm{~Hz}, 2 \mathrm{H}), 3.59(\mathrm{~s}, 6 \mathrm{H}) ;{ }^{13} \mathrm{C}$ NMR $\left(\mathrm{CDCl}_{3}, 75 \mathrm{MHz}\right) \delta 167.4(\mathrm{CO}), 143.2(\mathrm{C}), 131.4(\mathrm{CH}), 130.1(\mathrm{CH}), 129.8(\mathrm{CH}), 129.3(\mathrm{C}), 127.1$ $(\mathrm{CH}), 51.8\left(\mathrm{CH}_{3}\right)$; IR $v_{\max }$ (thin film) 2924, 1727, 1598, 1574, 1431, 1259, 1189, 1081, 1049, 962, 799, 752, $706 \mathrm{~cm}^{-1}$; MS, m/z (EI, 70eV) 270 (M+e, 23\%), 239 (13), 211 (100), 197 (27), 180 (30), 152 (27), 139 (20), 76 (23); HRMS, Found: $\mathrm{M}^{+\bullet}, 270.0892 . \mathrm{C}_{16} \mathrm{H}_{14} \mathrm{O}_{4}$ requires $\mathrm{M}^{+\bullet}, 270.0892$.

Concentration of fraction B $\left(R_{\mathrm{f}}=0.15\right.$ in $7: 3 \mathrm{v} / \mathrm{v}$ hexane-ethyl acetate $)$ afforded compound $\mathbf{4 7}^{20}$ (28\% from 45) as a white crystalline solid, $\mathrm{mp}=291-293{ }^{\circ} \mathrm{C}$ (lit. $\left.{ }^{20} \mathrm{mp}=291-292{ }^{\circ} \mathrm{C}\right) .{ }^{1} \mathrm{H} \mathrm{NMR}$ $\left[\left(\mathrm{CD}_{3}\right)_{2} \mathrm{SO}, 300 \mathrm{MHz}\right] \delta 11.69$ (br. s, $\left.1 \mathrm{H}\right), 8.50$ (d, $\left.J=7.7 \mathrm{~Hz}, 1 \mathrm{H}\right), 8.38$ (d, $\left.J=7.4 \mathrm{~Hz}, 1 \mathrm{H}\right), 8.31$ (dd, $J$ $=1.1$ and $7.8 \mathrm{~Hz}, 1 \mathrm{H}), 7.84(\mathrm{dt}, J=1.1$ and $7.8 \mathrm{~Hz}, 1 \mathrm{H}), 7.63(\mathrm{dt}, J=1.1$ and $7.4 \mathrm{~Hz}, 1 \mathrm{H}), 7.48(\mathrm{dt}, J=$ 1.1 and $7.8 \mathrm{~Hz}, 1 \mathrm{H}), 7.35(\mathrm{dd}, J=1.1$ and $7.8 \mathrm{~Hz}, 1 \mathrm{H}), 7.25(\mathrm{dt}, J=1.1$ and $7.8,1 \mathrm{H})$; ${ }^{13} \mathrm{C}$ NMR $\left[\left(\mathrm{CD}_{3}\right)_{2} \mathrm{SO}, 125 \mathrm{MHz}\right] \delta 160.8(\mathrm{CO}), 136.6(\mathrm{C}), 134.3(\mathrm{C}), 132.8(\mathrm{CH}), 129.6(\mathrm{CH}), 128.0(\mathrm{CH}), 127.5$ $(\mathrm{CH}), 125.7(\mathrm{C}), 123.3(\mathrm{CH}), 122.6(\mathrm{CH}), 122.3(\mathrm{CH}), 117.6(\mathrm{C}), 116.1(\mathrm{CH})$; IR $v_{\max }(\mathrm{KBr}) 2923,1660$, 1632, 1607, 1557, 1511, 1469, 1424, 1369, 1361, 1154, 1037, 749, $726 \mathrm{~cm}^{-1}$; MS, m/z (EI, $\left.70 \mathrm{eV}\right) 195$ $\left(\mathrm{M}^{+\bullet}, 100 \%\right), 167$ (23), 166 (13), 140 (10), 139 (14); HRMS, Found: $\mathrm{M}^{+\bullet}, 195.0684 . \mathrm{C}_{13} \mathrm{H}_{9} \mathrm{NO}$ requires $\mathrm{M}^{+\bullet}, 195.0684$.

4,2'-Dinitro-biphenyl-2-carboxylic Acid Methyl Ester (49). Cross-coupling of 1-bromo-2nitrobenzene $(\mathbf{1}, \mathrm{R}=\mathrm{H})$ with commercially available compound $\mathbf{4 8}$ in the same manner as described above for the preparation of compound $\mathbf{2 1}$ gave a yellow oil on work-up. Subjection of this material to flash chromatography flash chromatography $(100: 3 \rightarrow 100: 8 \mathrm{v} / \mathrm{v}$ hexane-ethyl acetate gradient elution) afforded, after concentration of the appropriate fractions $\left(R_{\mathrm{f}}=0.4\right.$ in 55:25:10 v/v/v hexane- $\mathrm{CH}_{2} \mathrm{Cl}_{2}-\mathrm{ethyl}$ acetate), compound $49(83 \%)$ as a light-yellow solid, $\mathrm{mp}=141-142{ }^{\circ} \mathrm{C} .{ }^{1} \mathrm{H} \mathrm{NMR}\left(\mathrm{CDCl}_{3}, 300 \mathrm{MHz}\right)$ $\delta 8.94(\mathrm{~d}, J=2.5 \mathrm{~Hz}, 1 \mathrm{H}), 8.45(\mathrm{dd}, J=1.5$ and $8.4 \mathrm{~Hz}, 1 \mathrm{H}), 8.24(\mathrm{dd}, J=1.4$ and $8.4 \mathrm{~Hz}, 1 \mathrm{H}), 7.72(\mathrm{dt}, J$ $=1.5$ and $7.6 \mathrm{~Hz}, 1 \mathrm{H}), 7.63(\mathrm{dt}, J=1.5$ and $7.8 \mathrm{~Hz}, 1 \mathrm{H}), 7.46(\mathrm{~d}, J=8.4 \mathrm{~Hz}, 1 \mathrm{H}), 7.26(\mathrm{dd}, J=1.5$ and $7.6 \mathrm{~Hz}, 1 \mathrm{H}), 3.75(\mathrm{~s}, 3 \mathrm{H}) ;{ }^{13} \mathrm{C} \mathrm{NMR}\left(\mathrm{CDCl}_{3}, 125 \mathrm{MHz}\right) \delta 164.6(\mathrm{C}), 147.4$ (C), 147.3 (C), 146.9 (C), $135.3(\mathrm{C}), 133.4(\mathrm{CH}), 131.3(\mathrm{CH}), 130.7(\mathrm{CH}), 129.9(\mathrm{C}), 129.4(\mathrm{CH}), 126.7(\mathrm{CH}), 125.7(\mathrm{CH}), 124.5$ $(\mathrm{CH}), 52.8\left(\mathrm{CH}_{3}\right)$; IR $v_{\max }(\mathrm{KBr}) 3098,2957,2853,1731,1607,1524,1439,1350,1255,1124,794 \mathrm{~cm}^{-1}$; MS, $m / z$ (EI, 70eV) $302\left(\mathrm{M}^{+\bullet}, 2 \%\right), 301$ (17), 271 (2), 256 (100), 201 (56), 139 (30); HRMS, Found: M $^{+\bullet}$, 302.0542. $\mathrm{C}_{14} \mathrm{H}_{10} \mathrm{~N}_{2} \mathrm{O}_{6}$ requires $\mathrm{M}^{+\bullet}, 302.0539$.

8-Amino-6(5H)-phenanthridinone (50). A magnetically stirred mixture of compound 49 (655 $\mathrm{mg}$, $2.17 \mathrm{mmol})$ and $10 \% \mathrm{Pd}$ on carbon $\mathrm{C}(328 \mathrm{mg})$ in methanol $(5 \mathrm{~mL})$ was exposed to dihydrogen $(1 \mathrm{~atm}$.). After stirring at $18{ }^{\circ} \mathrm{C}$ for $2 \mathrm{~h}$, the reaction mixture was filtered through a short pad of Celite ${ }^{\mathrm{TM}}$ and the filtrate was concentrated under reduced pressure. The ensuing light-yellow oil was subjected to flash chromatography (100:5 v/v methanol/triethylamine elution) and thus affording, after concentration of the appropriate fractions $\left(R_{\mathrm{f}=} 0.5\right.$ in 8:3:1 v/v/v ethyl acetate/hexane/methanol), compound 50 (283 mg, 62\%) 
as a light-yellow solid, mp could not be determined, sublimation: $>217{ }^{\circ} \mathrm{C} .{ }^{1} \mathrm{H}$ NMR $\left[\left(\mathrm{CD}_{3}\right)_{2} \mathrm{SO}, 300\right.$ $\mathrm{MHz}] \delta 11.40(\mathrm{~s}, 1 \mathrm{H}), 8.14(\mathrm{~m}, 2 \mathrm{H}), 7.43(\mathrm{~m}, 1 \mathrm{H}), 7.28(\mathrm{~m}, 2 \mathrm{H}), 7.15(\mathrm{~m}, 1 \mathrm{H}), 7.08(\mathrm{dd}, J=1.5$ and 8.7 $\mathrm{Hz}, 1 \mathrm{H}), 5.73$ (s, 2H); ${ }^{13} \mathrm{C} \mathrm{NMR}\left[\left(\mathrm{CD}_{3}\right)_{2} \mathrm{SO}, 75 \mathrm{MHz}\right] \delta 160.9$ (C), 148.9 (C), 134.6 (C), 127.0 (C), $126.8(\mathrm{CH}), 123.6(\mathrm{CH}), 122.9(\mathrm{C}), 121.9(\mathrm{CH}), 121.5(\mathrm{CH}), 120.1(\mathrm{CH}), 118.7(\mathrm{C}), 115.7(\mathrm{CH}), 109.2$ $(\mathrm{CH})$; IR $v_{\max }(\mathrm{KBr}) 3442,3384,2923,2852,1729,1663,1558,1516,1485,1372,1305,1082,896,826$, $744 \mathrm{~cm}^{-1}$; MS, m/z (EI, 70eV) $210\left(\mathrm{M}^{+\bullet}, 100 \%\right), 181$ (21), 154 (9), 85 (16), 71 (21), 57 (29); HRMS, Found: $\mathrm{M}^{+\bullet}, 210.0790 . \mathrm{C}_{13} \mathrm{H}_{10} \mathrm{~N}_{2} \mathrm{O}$ requires $\mathrm{M}^{+\bullet}, 210.0793$.

4-Methoxy-2'-nitro-biphenyl-2-carboxylic Acid Methyl Ester (52). Cross-coupling of 1-bromo-2nitrobenzene $(\mathbf{1}, \mathrm{R}=\mathrm{H})$ with commercially available compound $\mathbf{5 1}$ in the same manner as described above for the preparation of compound $\mathbf{2 1}$ gave a yellow oil on work-up. Subjection of this material to flash chromatography $(100: 2 \rightarrow 100: 6 \mathrm{v} / \mathrm{v}$ hexane-ethyl acetate gradient elution) afforded, after concentration of the appropriate fractions $\left(R_{\mathrm{f}}=0.4\right.$ in 55:25:5 v/v/v hexane- $\mathrm{CH}_{2} \mathrm{Cl}_{2}$-ethyl acetate), compound $\mathbf{5 2}(36 \%)$ as a light-yellow solid, $\mathrm{mp}=102-103.5 .{ }^{1} \mathrm{H} \mathrm{NMR}\left(\mathrm{CDCl}_{3}, 300 \mathrm{MHz}\right) \delta 8.06(\mathrm{dd}, J=1.4$ and 8.1 Hz, $1 \mathrm{H})$, $7.60(\mathrm{~m}, 2 \mathrm{H}), 7.50(\mathrm{dt}, J=2.0$ and $7.6 \mathrm{~Hz}, 1 \mathrm{H}), 7.27(\mathrm{dd}, J=1.5$ and $7.1 \mathrm{~Hz}, 1 \mathrm{H}), 7.14(\mathrm{~m}, 2 \mathrm{H}), 3.90$ (s,

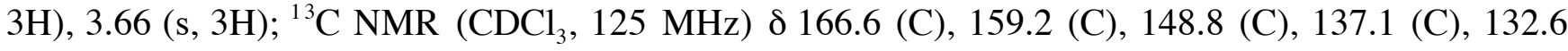
$(\mathrm{CH}), 132.0(\mathrm{C}), 131.9(\mathrm{CH}), 131.3(\mathrm{CH}), 129.7(\mathrm{C}), 128.1(\mathrm{CH}), 124.0(\mathrm{CH}), 118.3(\mathrm{CH}), 115.4(\mathrm{CH})$, $55.6\left(\mathrm{CH}_{3}\right), 52.2\left(\mathrm{CH}_{3}\right)$; IR $v_{\max }(\mathrm{KBr}) 2952,2852,1723,1609,1573,1522,1468,1434,1349,1222$, 1077, 1048, 855, 787, $755 \mathrm{~cm}^{-1}$; MS, m/z (EI, 70eV) $287\left(\mathrm{M}^{+\bullet}, 23 \%\right), 242$ (38), 241 (100), 226 (52), 211 (28), 139 (26), 127 (20); HRMS, Found: $\mathrm{M}^{+\bullet}, 287.0793 . \mathrm{C}_{15} \mathrm{H}_{13} \mathrm{NO}_{5}$ requires $\mathrm{M}^{+\bullet}, 287.0794$.

8-Methoxy-6(5H)-phenanthridinone (53). A magnetically stirred mixture of compound 52 (155 mg, $0.54 \mathrm{mmol})$ and $10 \% \mathrm{Pd}$ on carbon $\mathrm{C}(39 \mathrm{mg})$ in methanol $(4 \mathrm{~mL})$ was exposed to dihydrogen $(1 \mathrm{~atm}$.). After stirring at $18{ }^{\circ} \mathrm{C}$ for $1 \mathrm{~h}$, the reaction mixture was filtered through a short pad of Celite ${ }^{\mathrm{TM}}$ and the filtrate was concentrated under reduced pressure. The ensuing light-yellow oil was subjected to flash chromatography 100:5 v/v methanol/triethylamine elution) thus affording, after concentration of the appropriate fractions $\left(R_{\mathrm{f}} 0.4\right.$ in 80:55:25 v/v/v ethyl acetate/hexane/ $\left.\mathrm{CH}_{2} \mathrm{Cl}_{2}\right)$, compound $\mathbf{5 3}(104 \mathrm{mg}, 85 \%)$ as a light-yellow solid, mp could not be determined, sublimation: $>163{ }^{\circ} \mathrm{C} .{ }^{1} \mathrm{H}$ NMR $\left[\left(\mathrm{CD}_{3}\right)_{2} \mathrm{SO}, 300\right.$ $\mathrm{MHz}] \delta 11.39(\mathrm{~s}, 1 \mathrm{H}), 8.50(\mathrm{~d}, J=8.9 \mathrm{~Hz}, 1 \mathrm{H}), 8.41(\mathrm{~d}, J=7.8 \mathrm{~Hz}, 1 \mathrm{H}), 7.77(\mathrm{~m}, 2 \mathrm{H}), 7.57$ (t, $J=7.8 \mathrm{~Hz}$, $1 \mathrm{H}), 7.46(\mathrm{~m}, 1 \mathrm{H}), 7.34(\mathrm{t}, J=7.8 \mathrm{~Hz}, 1 \mathrm{H}), 3.93(\mathrm{~s}, 3 \mathrm{H}) ;{ }^{13} \mathrm{C} \mathrm{NMR}\left[\left(\mathrm{CD}_{3}\right)_{2} \mathrm{SO}, 75 \mathrm{MHz}\right] \delta 159.1(\mathrm{C})$, $156.4(\mathrm{C}), 135.9(\mathrm{C}), 128.8(\mathrm{CH}), 126.8(\mathrm{C}), 125.7(\mathrm{C}), 124.7(\mathrm{CH}), 122.7(\mathrm{CH}), 121.4(\mathrm{CH}), 117.4(\mathrm{C})$, $113.0(\mathrm{CH}), 108.5(\mathrm{CH}), 55.5\left(\mathrm{CH}_{3}\right)$ (one signal obscured or overlapping); IR $v_{\max }(\mathrm{KBr}) 2921,2847$, 1658, 1609, 1453, 1363, 1290, 1217, 1024, 867, 845, 818, $739 \mathrm{~cm}^{-1}$; MS, m/z (EI, 70eV) $225\left(\mathrm{M}^{+\bullet}, 100 \%\right)$, 210 (30), 196 (32), 182 (18), 154 (30), 153 (22), 57 (16); HRMS, Found: $\mathrm{M}^{+\bullet}, 225.0790 . \mathrm{C}_{14} \mathrm{H}_{11} \mathrm{NO}_{2}$ requires $\mathrm{M}^{+\bullet}, 225.0790$. 
2'-Nitro-biphenyl-2,5-dicarboxylic Acid Dimethyl Ester (55). Cross-coupling of 1-bromo-2nitrobenzene $(1, \mathrm{R}=\mathrm{H})$ with commercially available compound 54 in the same manner as described above for the preparation of compound $\mathbf{2 1}$ gave a yellow oil on work-up. Subjection of this material to flash chromatography (100:3 $\rightarrow$ 100:10 v/v hexane-ethyl acetate gradient elution) afforded, after concentration of the appropriate fractions $\left(R_{\mathrm{f}}=0.4 \mathrm{in} 55: 25: 10 \mathrm{v} / \mathrm{v} / \mathrm{v}\right.$ hexane $/ \mathrm{CH}_{2} \mathrm{Cl}_{2} /$ ethyl acetate), compound $\mathbf{5 5}(48 \%)$ as a light-yellow solid, $\mathrm{mp}=122.5-124{ }^{\circ} \mathrm{C} .{ }^{1} \mathrm{H} \mathrm{NMR}\left[\left(\mathrm{CD}_{3}\right)_{2} \mathrm{SO}, 300 \mathrm{MHz}\right] \delta 8.17(\mathrm{dd}, J=1.2$ and 8.1 $\mathrm{Hz}, 1 \mathrm{H}), 8.12$ (s, 2H), 7.83-7.60 (complex m, 2H), 7.69 (m, 1H), 7.42 (dd, $J=1.4$ and 7.6 Hz, 1H), 3.88 $(\mathrm{s}, 3 \mathrm{H}), 3.61(\mathrm{~s}, 3 \mathrm{H}) ;{ }^{13} \mathrm{C}$ NMR $\left[\left(\mathrm{CD}_{3}\right)_{2} \mathrm{SO}, 75 \mathrm{MHz}\right] \delta 166.0(\mathrm{C}), 165.8(\mathrm{C}), 147.9(\mathrm{C}), 140.4(\mathrm{C}), 136.4$ (C), $133.3(\mathrm{C}), 133.0(\mathrm{CH}), 132.3(\mathrm{C}), 131.4(\mathrm{CH}), 131.0(\mathrm{CH}), 130.6(\mathrm{CH}), 129.0(\mathrm{CH}), 128.7(\mathrm{CH})$, $124.2(\mathrm{CH}), 52.6\left(\mathrm{CH}_{3}\right), 52.4\left(\mathrm{CH}_{3}\right)$; IR $v_{\max }(\mathrm{KBr}) 2954,2850,1725,1613,1573,1525,1435,1349$, 1289, 1241, 1117, $749 \mathrm{~cm}^{-1}$; MS, m/z (EI, 70eV) 284 [(M- $\left.\left.\mathrm{CH}_{3} \mathrm{O} \cdot\right)^{+}, 19 \%\right], 269$ (100), 223 (23), 195 (170, 167 (13), 151 (16), 139 (25), 119 (9); HRMS, Found: $\left(\mathrm{M}-\mathrm{CH}_{3} \mathrm{O} \cdot\right)^{+}, 284.0561 . \mathrm{C}_{16} \mathrm{H}_{13} \mathrm{NO}_{6}$ requires $\left(\mathrm{M}-\mathrm{CH}_{3} \mathrm{O} \cdot\right)^{+}, 284.0559$.

6-Oxo-5,6-dihydrophenanthridine-9-carboxylic Acid Methyl Ester (56). A magnetically stirred mixture of compound 55 (253 mg, $0.80 \mathrm{mmol})$ and $10 \% \mathrm{Pd}$ on carbon (63 mg) in methanol (4 mL) was exposed to dihydrogen ( $1 \mathrm{~atm}$.). After stirring at $18{ }^{\circ} \mathrm{C}$ for $1 \mathrm{~h}$, the reaction mixture was filtered through a short pad of Celite ${ }^{\mathrm{TM}}$ and the filtrate was concentrated under reduced pressure. The ensuing residue was subjected to flash chromatography (100:5 v/v methanol/ triethylamine elution) thus affording, after concentration of the appropriate fractions $\left(R_{\mathrm{f}}=0.4\right.$ in 80:55:25 v/v/v ethyl acetate/hexane $\left./ \mathrm{CH}_{2} \mathrm{Cl}_{2}\right)$, compound 56 (108 mg, 53\%) as a white solid, mp could not be determined, sublimation: $>185{ }^{\circ} \mathrm{C} .{ }^{1} \mathrm{H}$ NMR [(CD $\left.)_{2} \mathrm{SO}, 300 \mathrm{MHz}\right] \delta 11.89(\mathrm{~s}, 1 \mathrm{H}), 8.96(\mathrm{~s}, 1 \mathrm{H}), 8.44(\mathrm{~d}, J=8.4 \mathrm{~Hz}, 2 \mathrm{H}), 8.13(\mathrm{~d}, J=8.4 \mathrm{~Hz}$, $1 \mathrm{H}), 7.54(\mathrm{t}, J=7.6 \mathrm{~Hz}, 1 \mathrm{H}), 7.39(\mathrm{~d}, J=8.4 \mathrm{~Hz}, 1 \mathrm{H}), 7.30(\mathrm{t}, J=7.6 \mathrm{~Hz}, 1 \mathrm{H}), 3.96(\mathrm{~s}, 3 \mathrm{H}) ;{ }^{13} \mathrm{C} \mathrm{NMR}$ $\left[\left(\mathrm{CD}_{3}\right)_{2} \mathrm{SO}, 75 \mathrm{MHz}\right] \delta 165.8(\mathrm{C}), 160.1(\mathrm{C}), 136.8(\mathrm{C}), 134.3(\mathrm{C}), 133.3(\mathrm{C}), 130.2(\mathrm{CH}), 128.7(\mathrm{C})$, $128.3(\mathrm{CH}), 127.6(\mathrm{CH}), 123.6(\mathrm{CH}), 123.4(\mathrm{CH}), 122.6(\mathrm{CH}), 117.0(\mathrm{C}), 116.3(\mathrm{CH}), 52.7\left(\mathrm{CH}_{3}\right)$; IR $v_{\max }(\mathrm{KBr}) 2918,2849,1728,1657,1611,1503,1434,1419,1287,1256,1026,856,748,724 \mathrm{~cm}^{-1}$; MS,

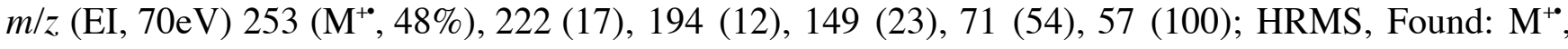
253.0739. $\mathrm{C}_{15} \mathrm{H}_{11} \mathrm{NO}_{3}$ requires $\mathrm{M}^{+\bullet}, 253.0739$.

6-(2-Nitrophenyl)benzo[1,3]dioxole-5-carboxylic Acid Methyl Ester (58). Cross-coupling of 1-bromo-2-nitrobenzene $(\mathbf{1}, \mathrm{R}=\mathrm{H})$ with bromo-ester $\mathbf{5 7 ^ { 2 1 }}$ was carried out in the same manner as described above for the preparation of compound $\mathbf{2 1}$ save for the use of a reaction temperature of $90{ }^{\circ} \mathrm{C}$ and a reaction time of $6 \mathrm{~h}$. Subjection of the yellow oil obtained on work-up to flash chromatography $(4: 1 \mathrm{v} / \mathrm{v}$ hexane-ethyl acetate elution) afforded two fractions, A and B.

Concentration of fraction $\mathrm{A}\left(R_{\mathrm{f}}=0.2\right)$ afforded a ca. $2: 1$ mixture of compound $\mathbf{5 8}(40 \%$ as determined by ${ }^{1} \mathrm{H}$ NMR analysis) and 2,2'-dinitrobiphenyl [product from reductive dimerization of compound $1(\mathrm{R}=\mathrm{H})$ ] as a yellow oil. Trituration (diethyl ether) of a small portion of this mixture afforded 
an analytically pure sample of compound 58 as a yellow crystalline solid, $\mathrm{mp}=133-134{ }^{\circ} \mathrm{C}$. ${ }^{1} \mathrm{H}$ NMR $\left(\mathrm{CDCl}_{3}, 300 \mathrm{MHz}\right) \delta 8.06(\mathrm{dd}, J=1.3$ and $8.1 \mathrm{~Hz}, 1 \mathrm{H}), 7.59(\mathrm{dt}, J=1.3$ and $8.1 \mathrm{~Hz}, 1 \mathrm{H}), 7.50(\mathrm{~s}, 1 \mathrm{H})$, $7.48(\mathrm{dt}, J=1.3$ and $8.1 \mathrm{~Hz}, 1 \mathrm{H}), 7.23(\mathrm{dd}, J=1.3$ and $8.1 \mathrm{~Hz}, 1 \mathrm{H}), 6.64(\mathrm{~s}, 1 \mathrm{H}), 6.08(\mathrm{~m}, 2 \mathrm{H}), 3.60$ (s, $3 \mathrm{H}) ;{ }^{13} \mathrm{C} \mathrm{NMR}\left(\mathrm{CDCl}_{3}, 125 \mathrm{MHz}\right) \delta 165.8$ (CO), 150.8 (C), 148.3 (C), 147.4 (C), 137.1 (C), 135.8 (C), 132.6 (CH), $131.3(\mathrm{CH}), 128.2(\mathrm{CH}), 124.0(\mathrm{CH}), 121.9(\mathrm{C}), 110.3(\mathrm{CH}), 110.1(\mathrm{CH}), 102.2\left(\mathrm{CH}_{2}\right), 52.0$ $\left(\mathrm{CH}_{3}\right)$; IR $v_{\max }(\mathrm{KBr}) 1721,1525,1505,1479,1374,1349,1255,1037 \mathrm{~cm}^{-1} ; \mathrm{MS}, \mathrm{m} / z$ (EI, $\left.70 \mathrm{eV}\right) 301$ ( $\mathrm{M}^{+\bullet}, 28 \%$ ), 255 (100), 240 (58); HRMS, Found: $\mathrm{M}^{+\bullet}$, 301.0588. $\mathrm{C}_{15} \mathrm{H}_{11} \mathrm{NO}_{6}$ requires $\mathrm{M}^{+\bullet}, 301.0586$. Anal.: Found C 59.85; H 3.65; N 4.70. Calculated for $\mathrm{C}_{15} \mathrm{H}_{11} \mathrm{NO}_{6} ; \mathrm{C} 59.80 ; \mathrm{H} 3.68 ; \mathrm{N} 4.65 \%$.

Concentration of fraction $\mathrm{B}\left(R_{\mathrm{f}}=0.1\right)$ afforded $\left[5,5^{\prime}\right]$ bi $\{$ benzo[1,3]dioxolyl\}-6,6'-dicarboxylic acid dimethyl ester ${ }^{21}$ (product from reductive dimerization of compound 57) (27\% as determined by ${ }^{1} \mathrm{H}$ NMR analysis) as a white crystalline solid, $\mathrm{mp}=159-160{ }^{\circ} \mathrm{C}$ (lit. $\left.{ }^{21} \mathrm{mp}=160-160.5^{\circ} \mathrm{C}\right) .{ }^{1} \mathrm{H} \mathrm{NMR}$ $\left(\mathrm{CDCl}_{3}, 300 \mathrm{MHz}\right) \delta 7.44(\mathrm{~s}, 2 \mathrm{H}), 6.58(\mathrm{~s}, 2 \mathrm{H}), 6.04(\mathrm{~m}, 4 \mathrm{H}), 3.61(\mathrm{~s}, 6 \mathrm{H}) ;{ }^{13} \mathrm{C} \mathrm{NMR}\left(\mathrm{CDCl}_{3}, 75 \mathrm{MHz}\right)$ ठ $166.3(\mathrm{CO}), 150.2(\mathrm{C}), 146.6(\mathrm{C}), 139.5(\mathrm{C}), 122.4(\mathrm{C}), 110.3(\mathrm{CH}), 109.8(\mathrm{CH}), 101.9\left(\mathrm{CH}_{2}\right), 51.8$ $\left(\mathrm{CH}_{3}\right)$; IR $v_{\max }(\mathrm{KBr}) 2953,2923,1727,1615,1504,1481,1435,1374,1353,1248,1123,1037,933 \mathrm{~cm}^{-1}$; MS, $m / z(E I, 70 \mathrm{eV}) 358\left(\mathrm{M}^{+\bullet}, 62 \%\right), 299$ (100), 284 (59), 268 (45); HRMS, Found: M $^{+\bullet}, 358.0691$. $\mathrm{C}_{18} \mathrm{H}_{14} \mathrm{O}_{8}$ requires $\mathrm{M}^{+\bullet}, 358.0689$.

Crinasidine $\{[1,3]$-Dioxolo[4,5-j]-6(5H)-phenanthridinone $\}$ (59). A solution of compound 58 $(11.0 \mathrm{mg}, 0.037 \mathrm{mmol})$ in ethanol $(10 \mathrm{~mL})$ was treated with $10 \% \mathrm{Pd}$ on carbon $(3 \mathrm{mg})$ and the resulting mixture stirred at $18{ }^{\circ} \mathrm{C}$ under an atmosphere of dihydrogen for $16 \mathrm{~h}$. The dihydrogen atmosphere was then replaced by nitrogen and the reaction mixture treated with sodium ethoxide $(2.0 \mathrm{~mL}$ of a $0.4 \mathrm{M}$ solution in ethanol). After $5 \mathrm{~h}$ at $18{ }^{\circ} \mathrm{C}$ the reaction mixture was concentrated under reduced pressure and the residue subjected to flash chromatography (90:9.5:0.5 v/v/v chloroform-methanol-5\% aq. ammonia elution). In this manner two fractions, $\mathrm{A}$ and $\mathrm{B}$, were obtained.

Concentration of fraction A $\left(R_{\mathrm{f}}=0.8\right)$ afforded compound $\mathbf{5 8}(3.2 \mathrm{mg}, 29 \%$ recovery) as yellow crystalline solid and identical, in all respects, with an authentic sample.

Concentration of fraction B $\left(R_{\mathrm{f}}=0.4\right)$ afforded compound $\mathbf{5 9}^{13}(5.8 \mathrm{mg}, 92 \%$ at $71 \%$ conversion) as white crystalline masses, $\mathrm{mp}=>350{ }^{\circ} \mathrm{C}$ (lit. ${ }^{13} \mathrm{mp}=>350{ }^{\circ} \mathrm{C}$ with partial sublimation at $340{ }^{\circ} \mathrm{C}$ ). ${ }^{1} \mathrm{H}$ NMR $\left[\left(\mathrm{CD}_{3}\right)_{2} \mathrm{SO}, 500 \mathrm{MHz}\right] \delta 11.62($ broad s, $1 \mathrm{H}), 8.29(\mathrm{~d}, J=8.0 \mathrm{~Hz}, 1 \mathrm{H}), 8.03(\mathrm{~s}, 1 \mathrm{H}), 7.63(\mathrm{~s}, 1 \mathrm{H})$, $7.42(\mathrm{t}, J=8.0 \mathrm{~Hz}, 1 \mathrm{H}), 7.32(\mathrm{~d}, J=8.0 \mathrm{~Hz}, 1 \mathrm{H}), 7.20(\mathrm{t}, J=8.0 \mathrm{~Hz}, 1 \mathrm{H}), 6.22(\mathrm{~s}, 2 \mathrm{H}) ;{ }^{3} \mathrm{C}$ NMR $\left[\left(\mathrm{CD}_{3}\right)_{2} \mathrm{SO}, 125 \mathrm{MHz}\right] \delta 160.2,152.2,148.1,135.9,131.2,128.8,123.2,122.1,121.1,117.7,115.9$, 105.1, 102.3, 101.6; IR $v_{\max }(\mathrm{KBr}) 1663,1584,1472,1430,1240,1040 \mathrm{~cm}^{-1}$; MS, m/z (EI, 70eV) 239 $\left(\mathrm{M}^{+\bullet}, 100 \%\right), 162$ (26), 91 (25); HRMS, Found: $\mathrm{M}^{+\bullet}$, 239.0581. $\mathrm{C}_{14} \mathrm{H}_{9} \mathrm{NO}_{3}$ requires $\mathrm{M}^{+\bullet}, 239.0582$. 


\section{References}

1. (a) Arnold, Z.; Holy, A. Collect. Czech. Chem. Commun. 1961, 26, 3059. (b) Robertson, I. R.; Sharp, J. T. Tetrahedron 1984, 40, 3095.

2. Curran, D. P.; Kuo, S. C. J. Org. Chem. 1984, 49, 2063.

3. Eisch, J. J.; Gopal, H.; Kuo, C. T. J. Org. Chem. 1978, 43, 2190.

4. Denton, D. A.; Smalley, R. K.; Suschitzky, H. J. Chem. Soc. 1964, 2421.

5. Nicolaou, K. C.; Dai, W.-M. J. Am. Chem. Soc. 1992, 114, 8908.

6. These compounds were prepared using modifications of the methods reported in the following papers: (a) Bovonsombat, P.; McNelis, E. Tetrahedron 1993, 49, 1525. (b) Piers, E.; Grierson, J. R.; Lau, K. C.; Nagakura, I. Can. J. Chem. 1982, 60, 210. Full details of these preparations will be reported in due course.

7. Curran, A. C. W.; Shepherd, R. G. J. Chem. Soc., Perkin Trans. 1 1976, 983.

8. Vieweg, H.; Wagner, G. Pharmazie 1979, 34, 785.

9. Hahn, W. E.; Lesiak, J. Pol. J. Chem. 1985, 59, 627.

10. Kar, G. K.; Karmakar, A. C.; Makur, A.; Ray, J. K. Heterocycles 1995, 41, 911.

11. Gilchrist, T. L.; Healy, A. M. M. Tetrahedron 1993, 49, 2543.

12. Kallianpur, C. S.; Merchant, J. R. J. Indian Chem. Soc. 1961, 38, 27.

13. Banwell, M. G.; Cowden, C. J. Aust. J. Chem. 1994, 47, 2235.

14. Bohlmann, F.; Fritz, G. Chem. Ber. 1976, 109, 3371.

15. Zhang, H.; Larock, R. C. J. Org. Chem. 2002, 67, 9318.

16. Clemo, G. R.; Felton, D. G. I. J. Chem. Soc. 1951, 671; Chem. Abstr. 1951, 45, 9060c.

17. Jousseaume, B.; Villeneuve, P. Tetrahedron 1989, 45, 1145.

18. Bailey, A. S.; Seager, J. F. J. Chem. Soc., Perkin Trans. 1 1974, 763.

19. Hennings, D. D.; Iwama, T.; Rawal, V. H. Org. Lett. 1999, 1, 1205.

20. The Aldrich Library of ${ }^{13} \mathrm{C}$ and ${ }^{1} \mathrm{H}$ NMR Spectra, Edition 1, Vol. 2; Aldrich Chemical Co.: Milwaukee, Wisconsin, 1993; p 1437A.

21. Brown, E.; Robin, J.-P.; Dhal, R. Tetrahedron 1982, 38, 2579. 
$300 \mathrm{MHz}^{1} \mathrm{HNMR}$ Spectrum in $\mathrm{CDCl}_{3}$
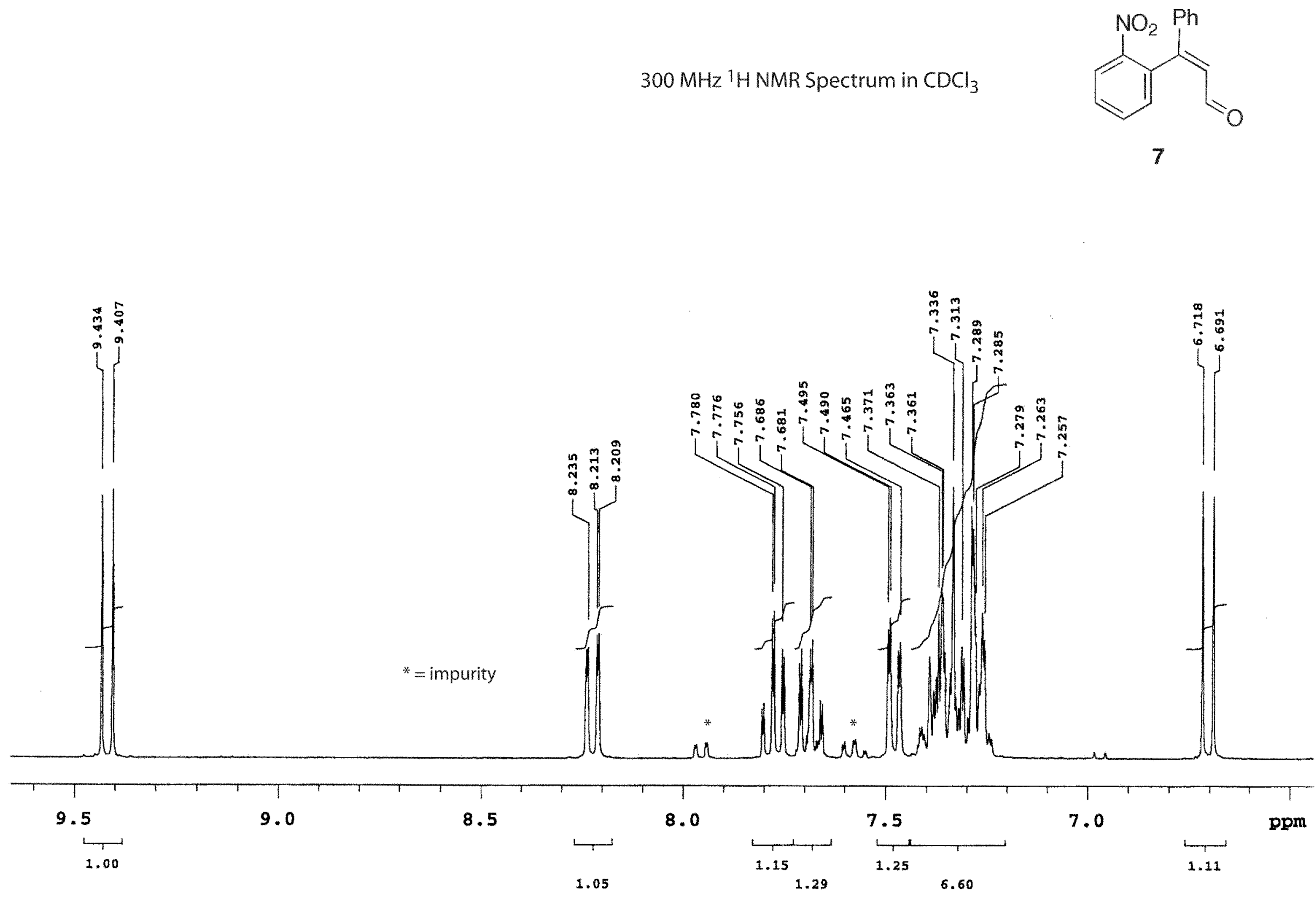
$75 \mathrm{MHz}{ }^{13} \mathrm{CNMR}$ Spectrum in $\mathrm{CDCl}_{3}$
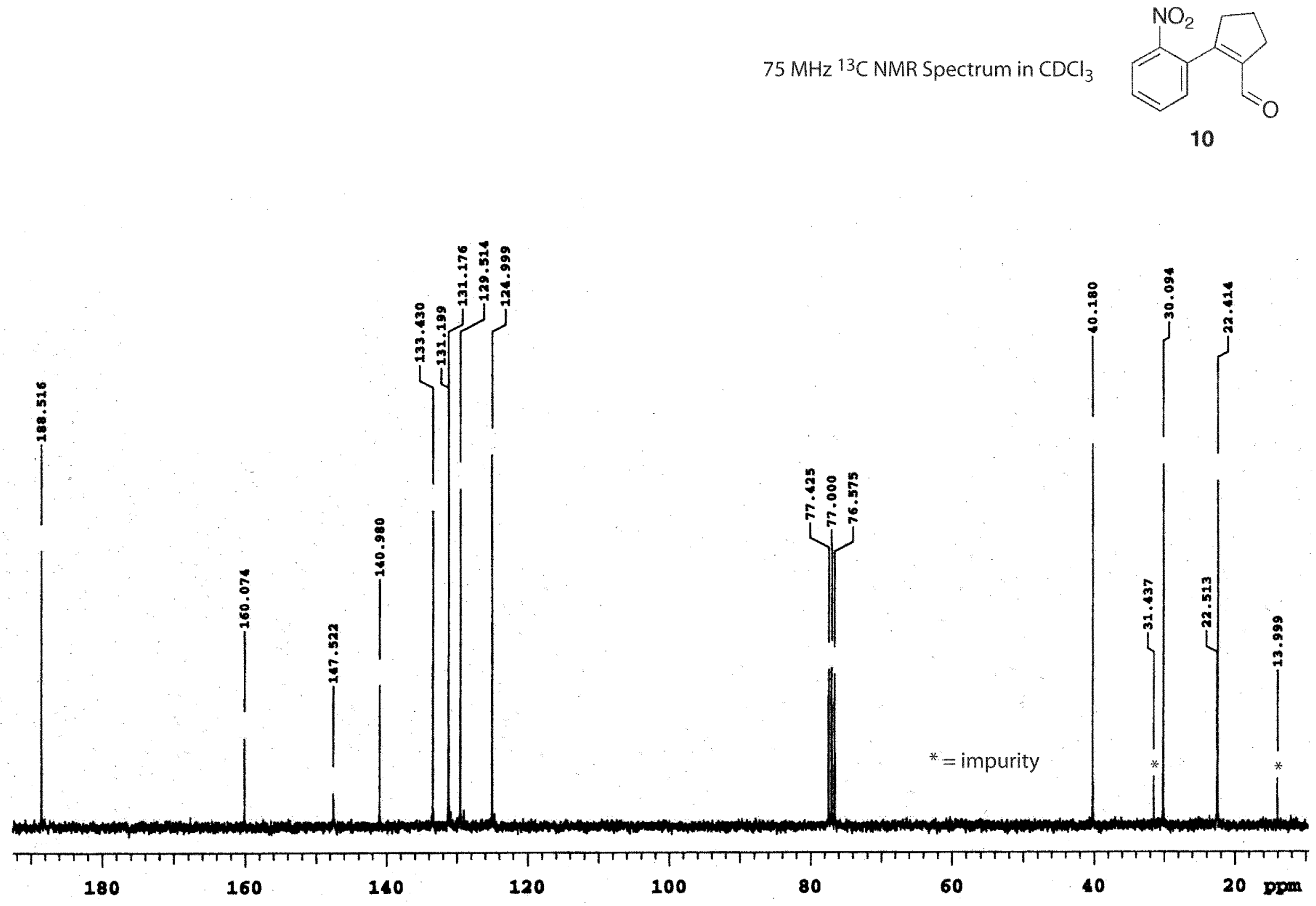
$75 \mathrm{MHz}^{13} \mathrm{CNMR}$ Spectrum in $\mathrm{CDCl}_{3}$

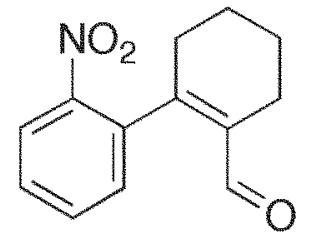

13

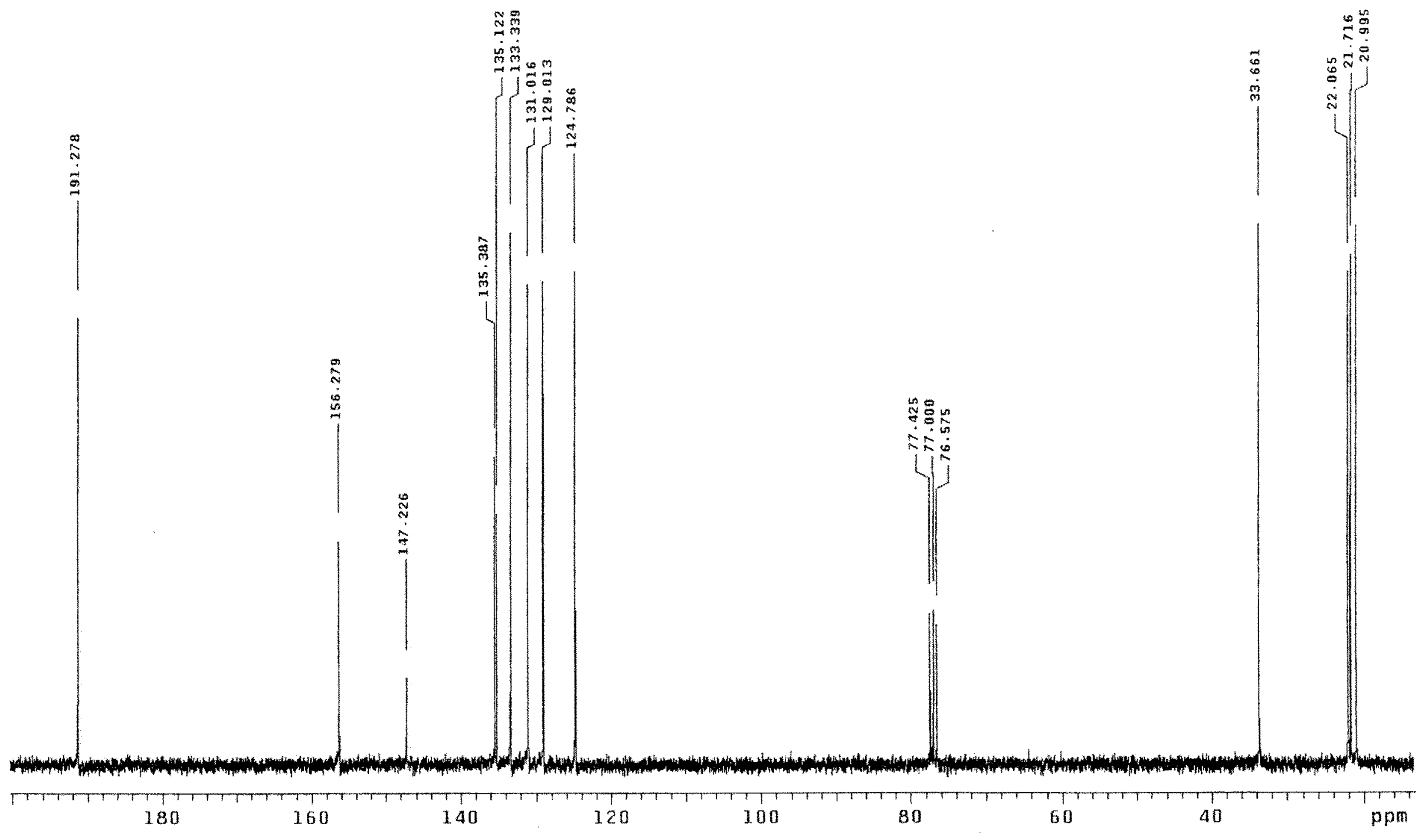


$75 \mathrm{MHz}^{13} \mathrm{C} \mathrm{NMR}$ spectrum in $\mathrm{CDCl}_{3}$
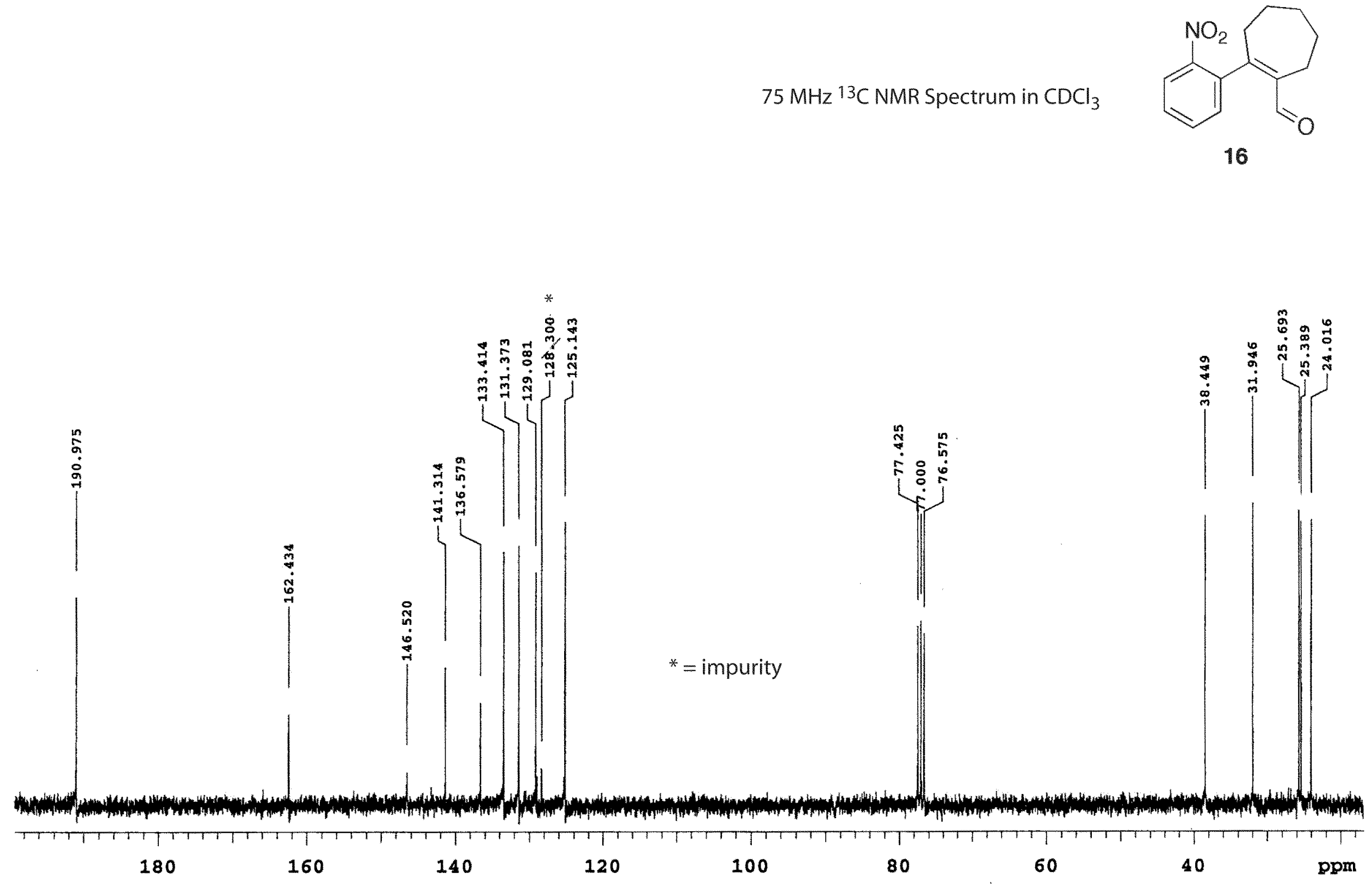
$75 \mathrm{MHz}^{13} \mathrm{C}$ NMR Spectrum in $\mathrm{CDCl}_{3}$

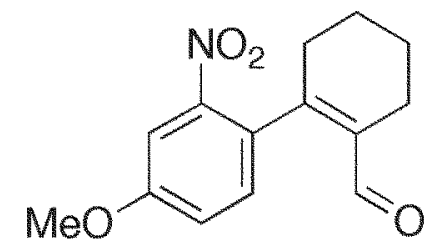

18

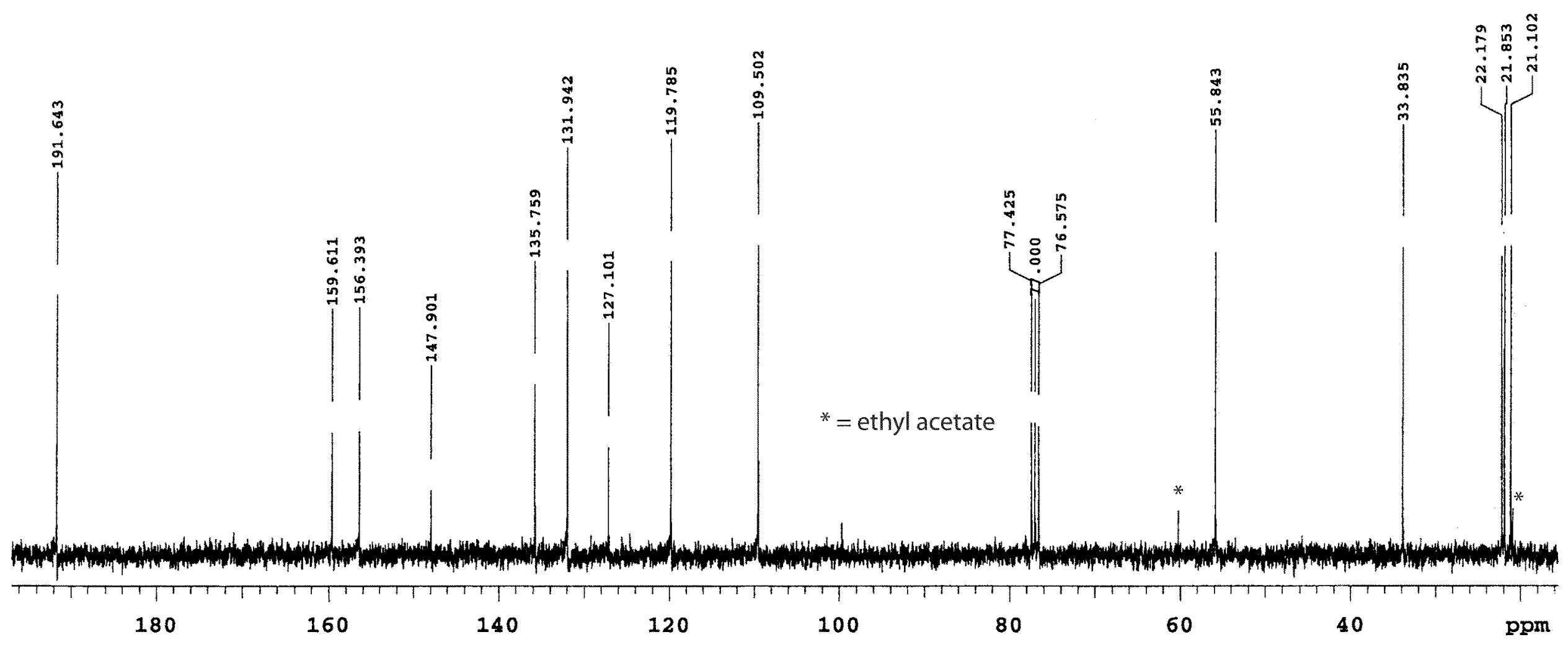




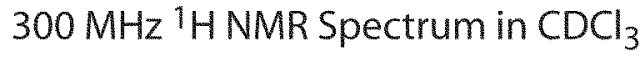

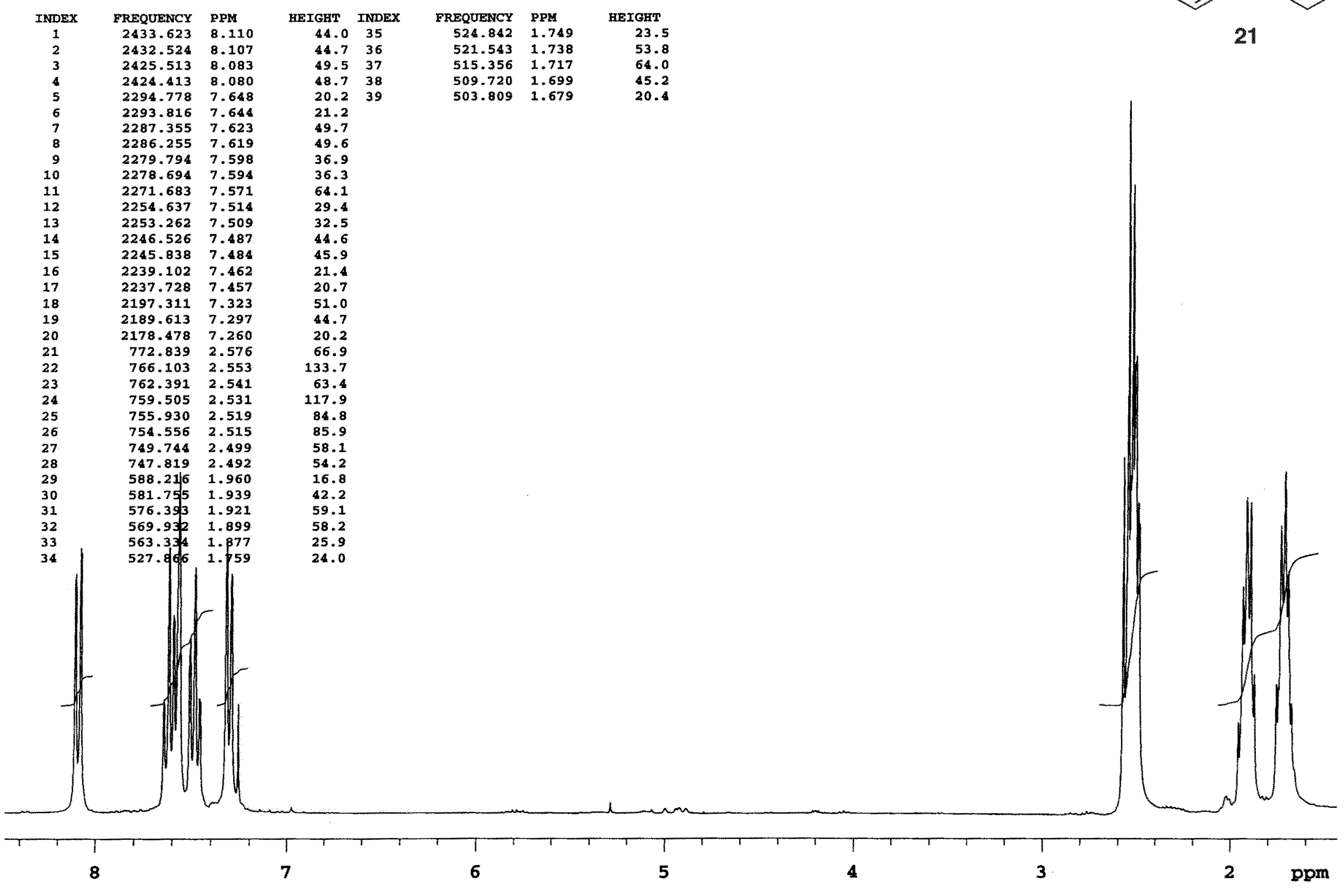




$\begin{array}{crl}\text { INDEX } & \text { FREQUENCY } & \text { PPM } \\ 1 & 2159.507 & 7.197 \\ 2 & 2158.957 & 7.195 \\ 3 & 768.578 & 2.561 \\ 4 & 761.842 & 2.539 \\ 5 & 757.992 & 2.526 \\ 6 & 751.806 & 2.505 \\ 7 & 496.650 & 1.655 \\ 8 & 492.124 & 1.640 \\ 9 & 487.037 & 1.623\end{array}$

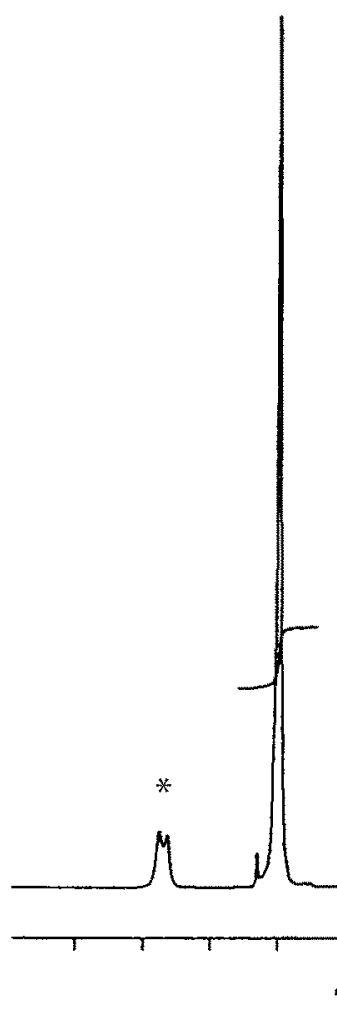

HEIGHT

87.7

86.3

123.9

67.1

141.5

110.4

106.5

$300 \mathrm{MHz}^{1} \mathrm{H}$ NMR Spectrum in $\mathrm{CDCl}_{3}$

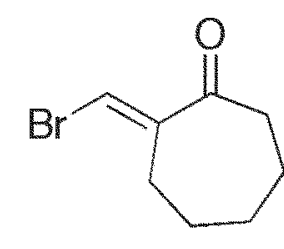

23

* = impurity

1

6

5
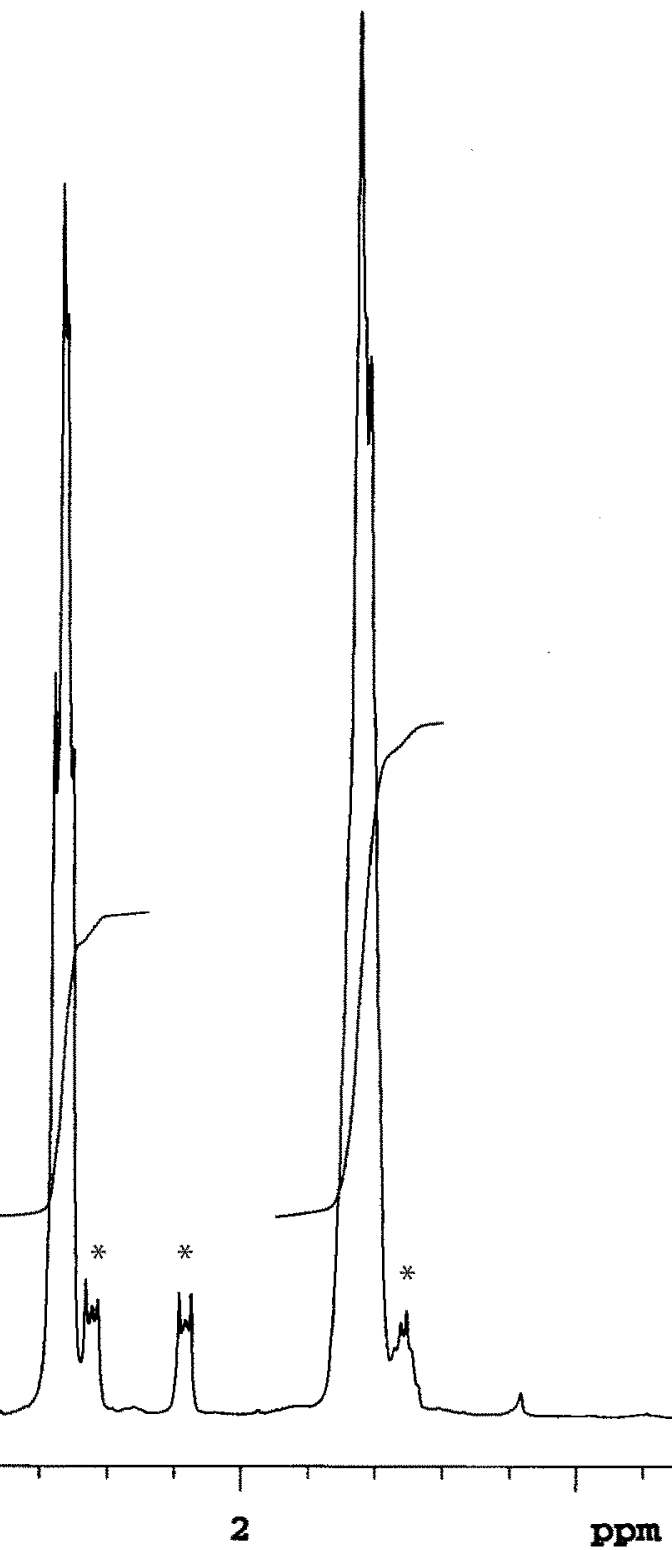


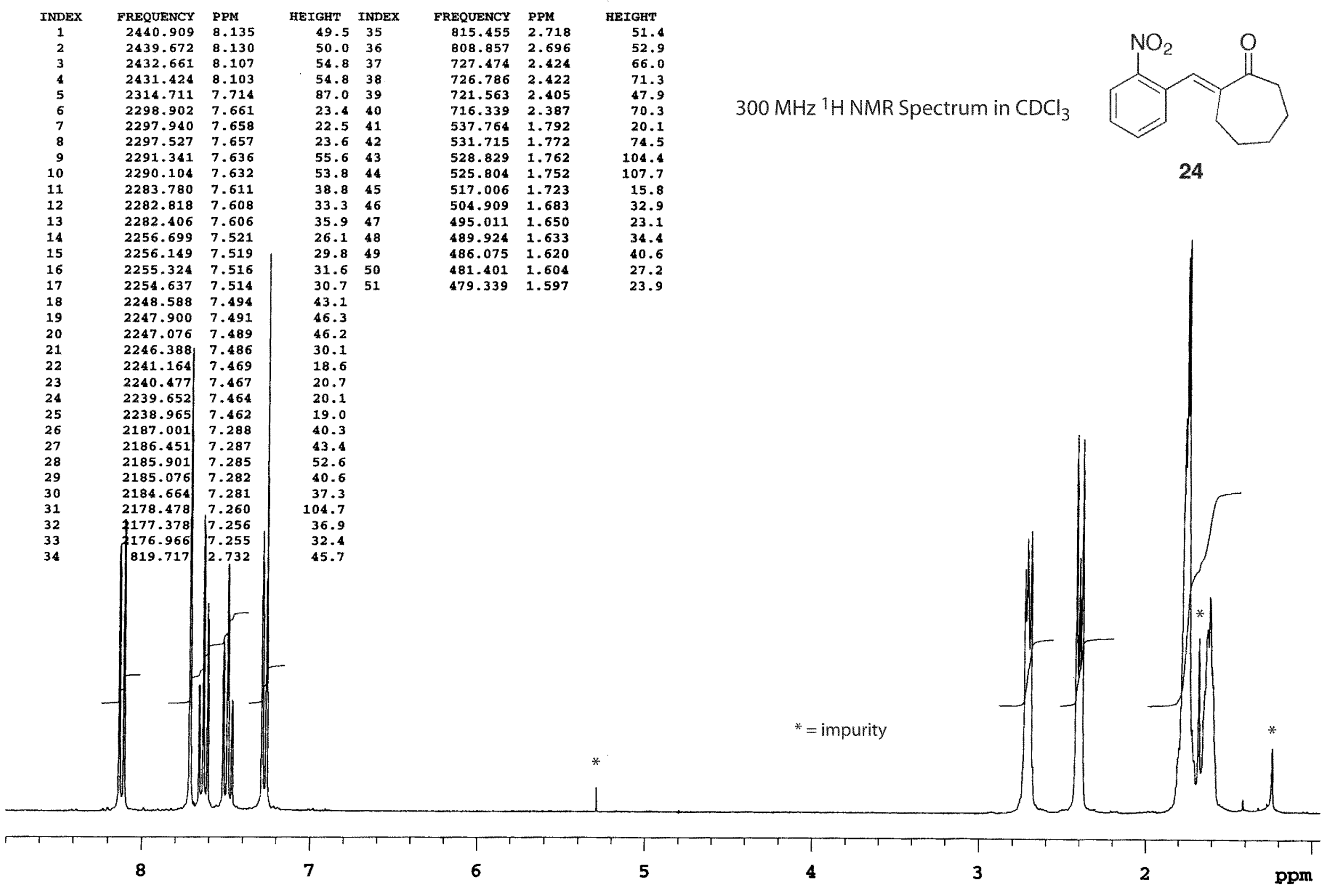




$\begin{array}{crlr}\text { INDEX } & \text { FREQUENCY } & \text { PPM } & \text { HEIGHT } \\ 1 & 2283.368 & 7.610 & 16.3 \\ 2 & 2280.756 & 7.601 & 16.6 \\ 3 & 2259.448 & 7.530 & 6.2 \\ 4 & 2257.523 & 7.523 & 11.2 \\ 5 & 2255.599 & 7.517 & 5.7 \\ 6 & 2178.478 & 7.260 & 16.5 \\ 7 & 2175.591 & 7.250 & 9.5 \\ 8 & 2166.793 & 7.221 & 18.2 \\ 9 & 2150.021 & 7.165 & 12.5 \\ 10 & 2147.409 & 7.156 & 11.5 \\ 11 & 2141.498 & 7.137 & 6.3 \\ 12 & 2138.749 & 7.128 & 6.1 \\ 13 & 1177.141 & 3.923 & 1.6 \\ 14 & 1168.343 & 3.894 & 139.7 \\ 15 & 772.702 & 2.575 & 16.0 \\ 16 & 769.815 & 2.565 & 8.8 \\ 17 & 766.103 & 2.553 & 33.7 \\ 18 & 759.367 & 2.531 & 23.2 \\ 19 & 757.168 & 2.523 & 8.4 \\ 20 & 589.591 & 1.965 & 3.0 \\ 21 & 582.992 & 1.943 & 6.5 \\ 22 & 577.768 & 1.925 & 8.5 \\ 23 & 575.156 & 1.917 & 5.1 \\ 24 & 571.170 & 1.903 & 8.6 \\ 25 & 568.145 & 1.893 & 2.2 \\ 26 & 564.571 & 1.881 & 3.6 \\ 27 & 530.203 & 1.767 & 3.9 \\ 28 & 527.179 & 1.757 & 3.0 \\ 29 & 524.017 & 1.746 & 8.0 \\ 30 & 517.693 & 1.725 & 9.0 \\ 31 & 511.920 & 1.706 & 5.9 \\ 32 & 509.720 & 1.699 & 2.8 \\ 33 & 505.871 & 1.686 & 3.1 \\ 34 & 503.259 & 1.677 & 3.3\end{array}$

$300 \mathrm{MHz}{ }^{1} \mathrm{H}$ NMR Spectrum in $\mathrm{CDCl}_{3}$

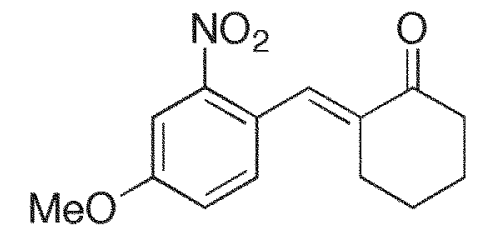

26
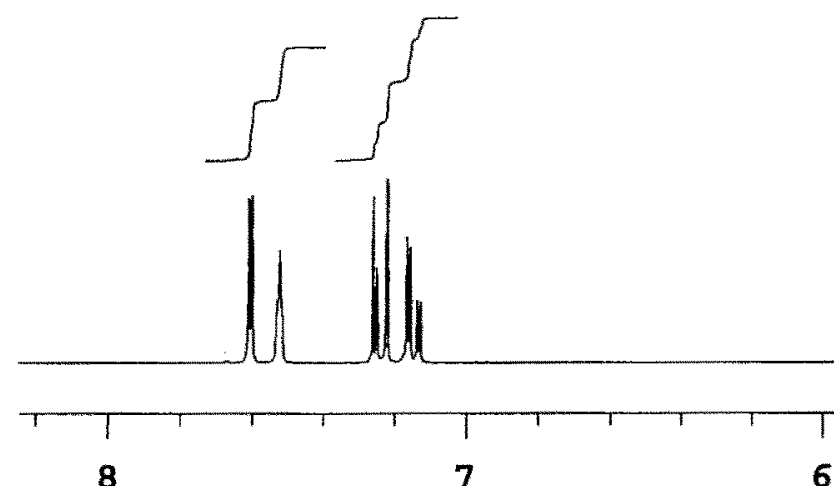

6

3

ppm 


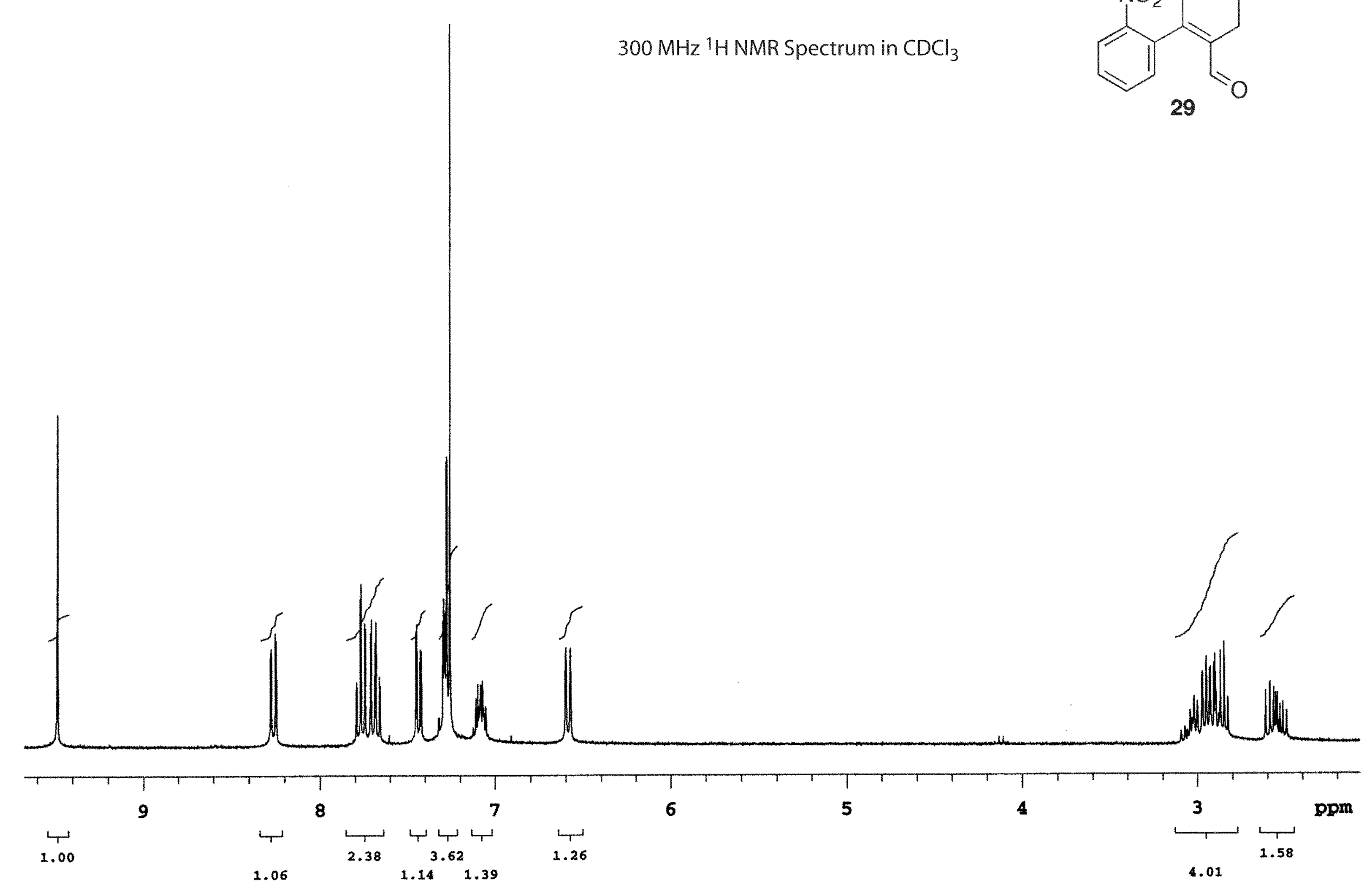


$300 \mathrm{MHz}^{1} \mathrm{HNMR}$ Spectrum in $\mathrm{CDCl}_{3}$
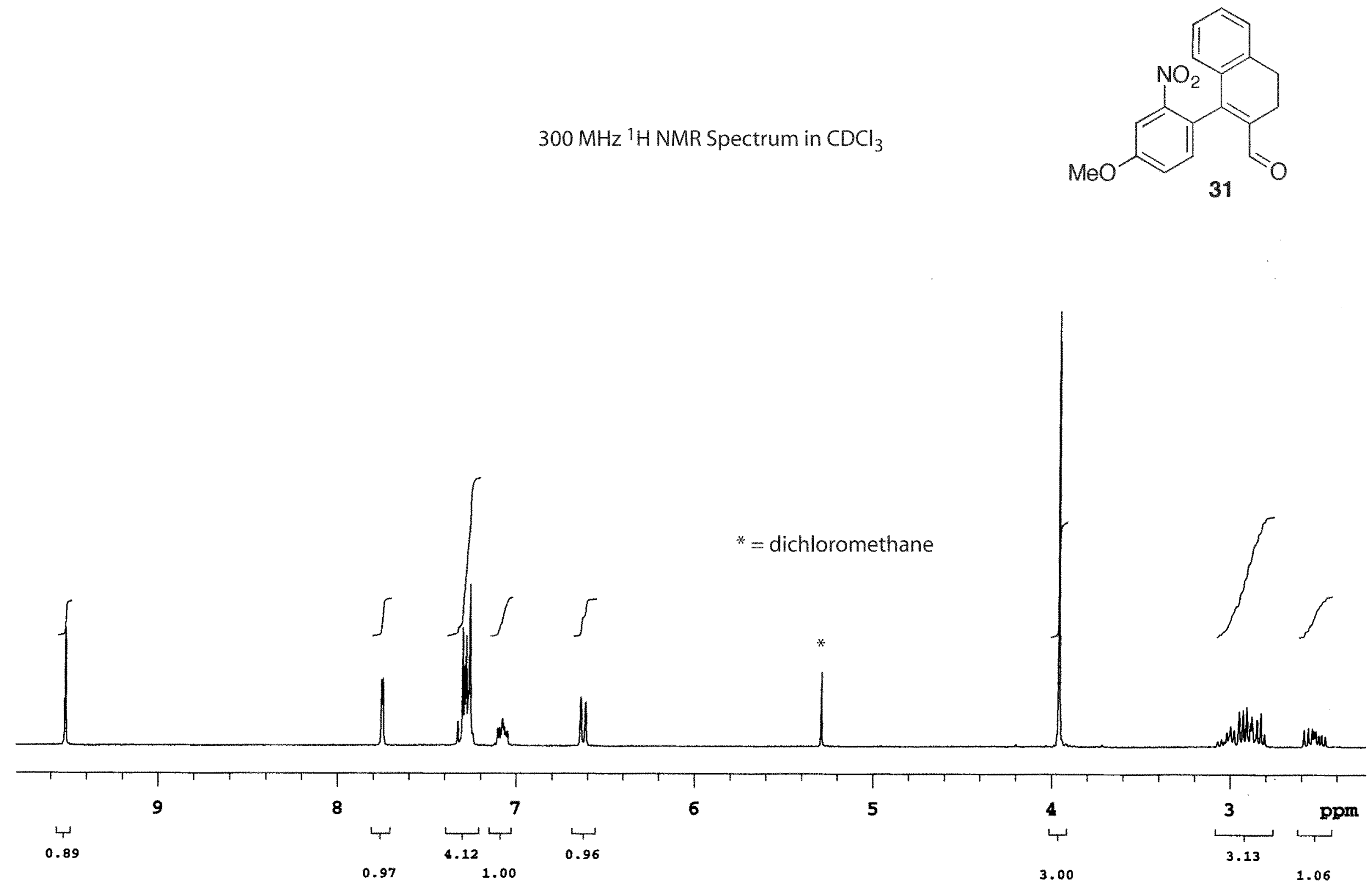
$300 \mathrm{MHz}^{1} \mathrm{H}$ NMR Spectrum in $\mathrm{CDCl}_{3}$

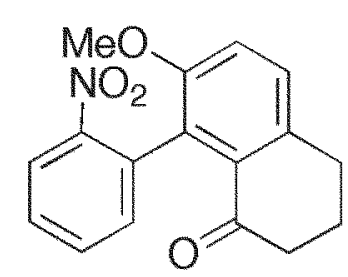

37

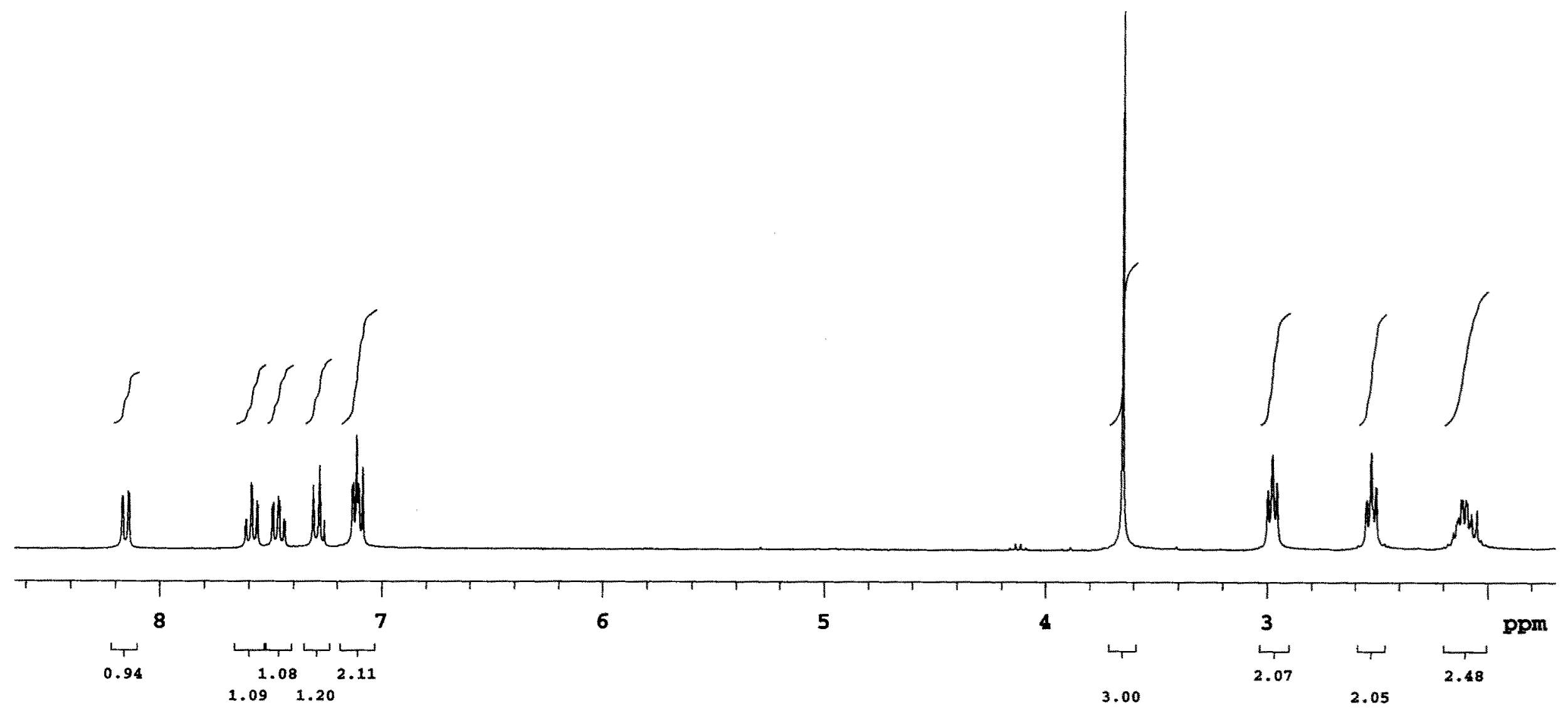


$300 \mathrm{MHz}^{1} \mathrm{H}$ NMR Spectrum in $\mathrm{CDCl}_{3}$
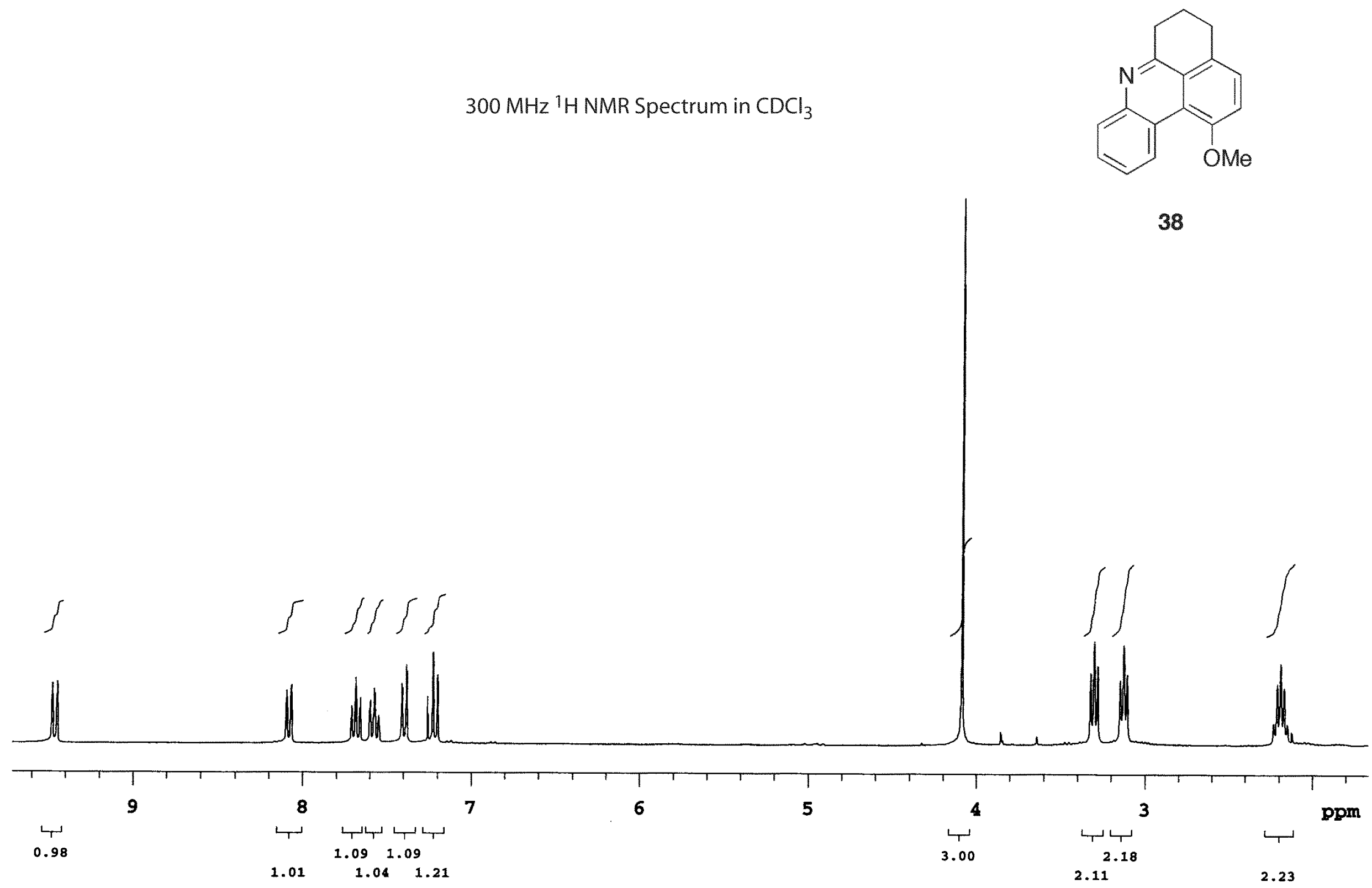
$300 \mathrm{MHz}^{1} \mathrm{H} \mathrm{NMR}$ spectrum in $\mathrm{CDCl}_{3}$

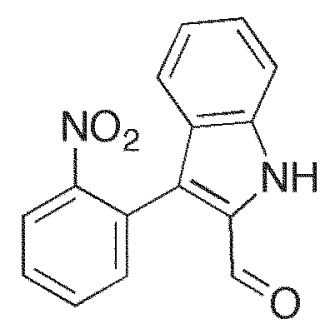

40
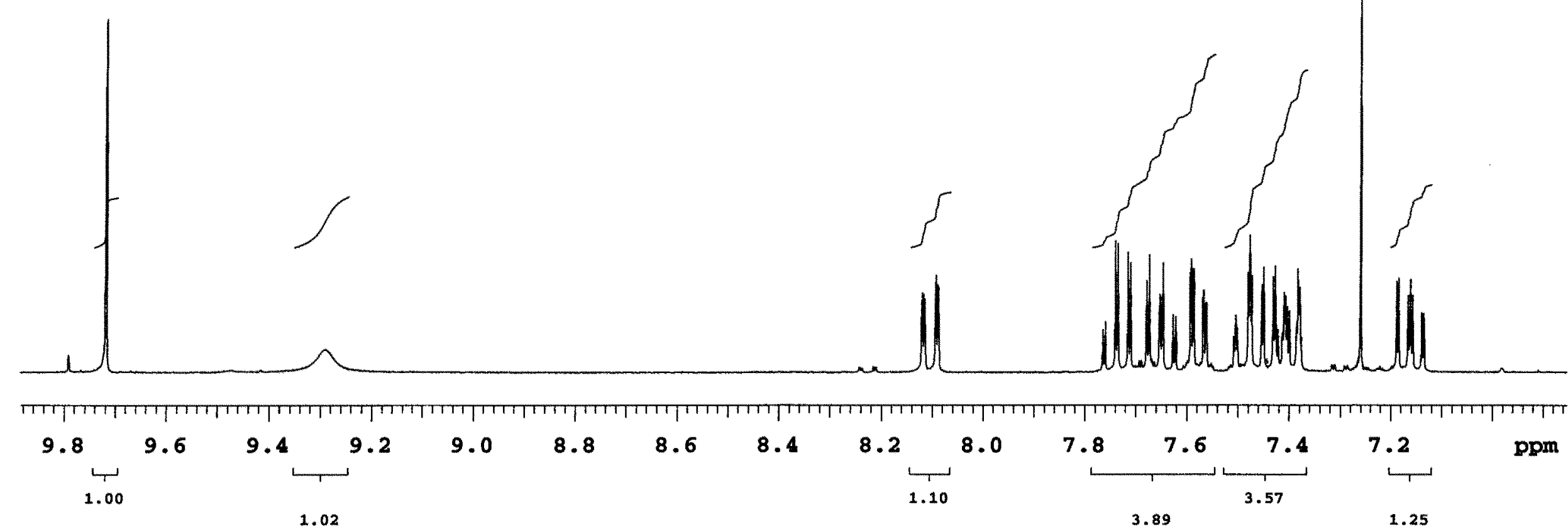
$300 \mathrm{MHz}{ }^{1} \mathrm{H}$ NMR Spectrum in $\left(\mathrm{CD}_{3}\right)_{2} \mathrm{CO}$

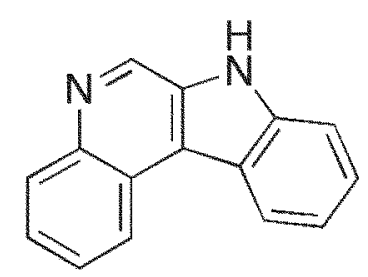

41

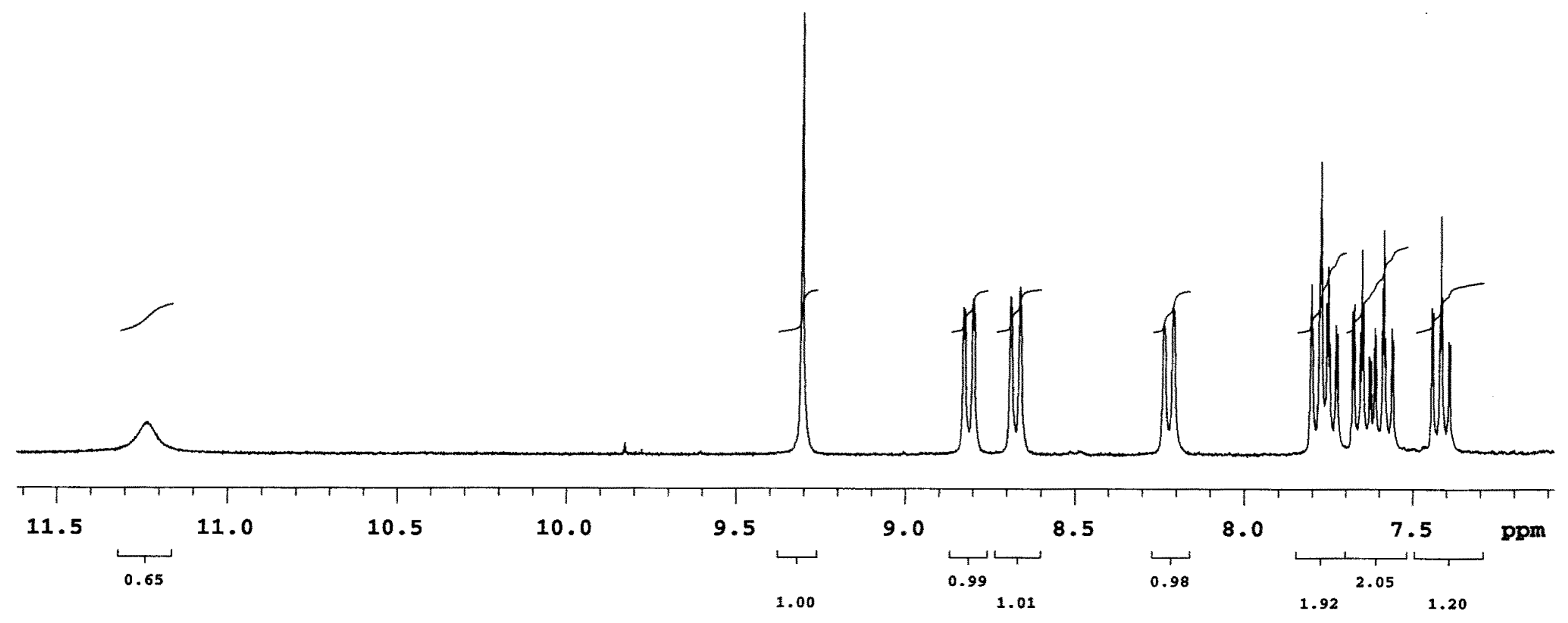


$75 \mathrm{MHz}^{1} \mathrm{H} \mathrm{NMR}$ Spectrum in $\mathrm{CDCl}_{3}$

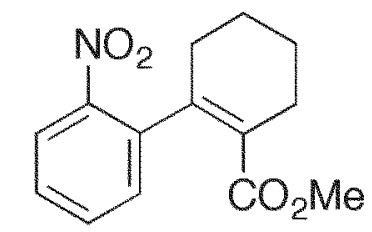

43

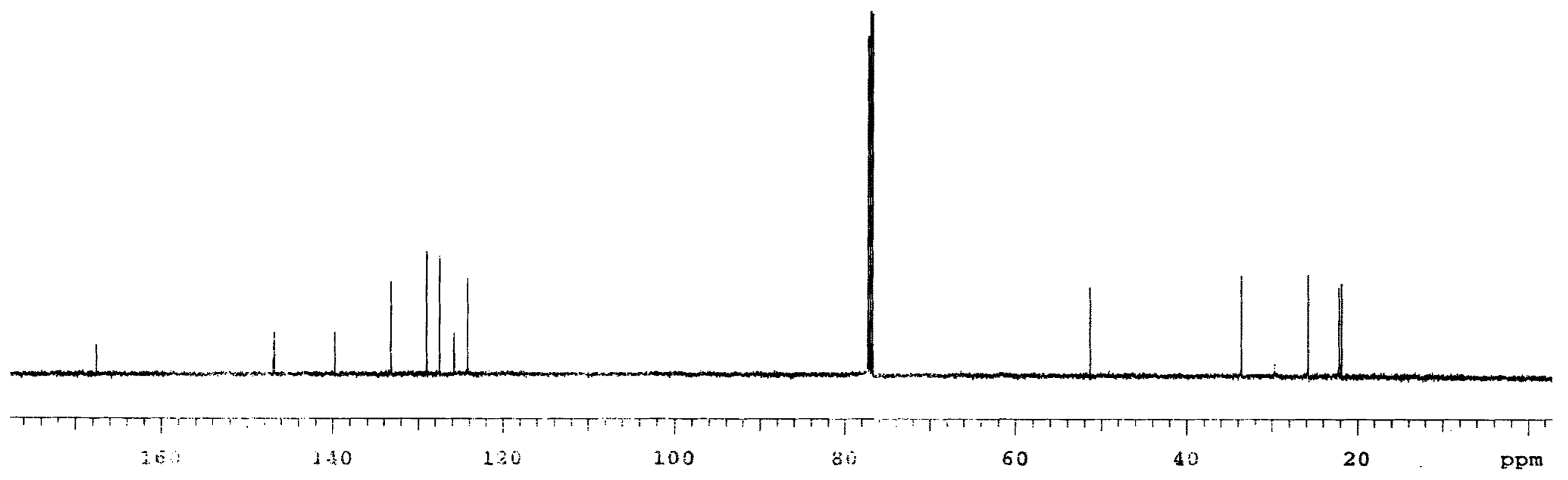




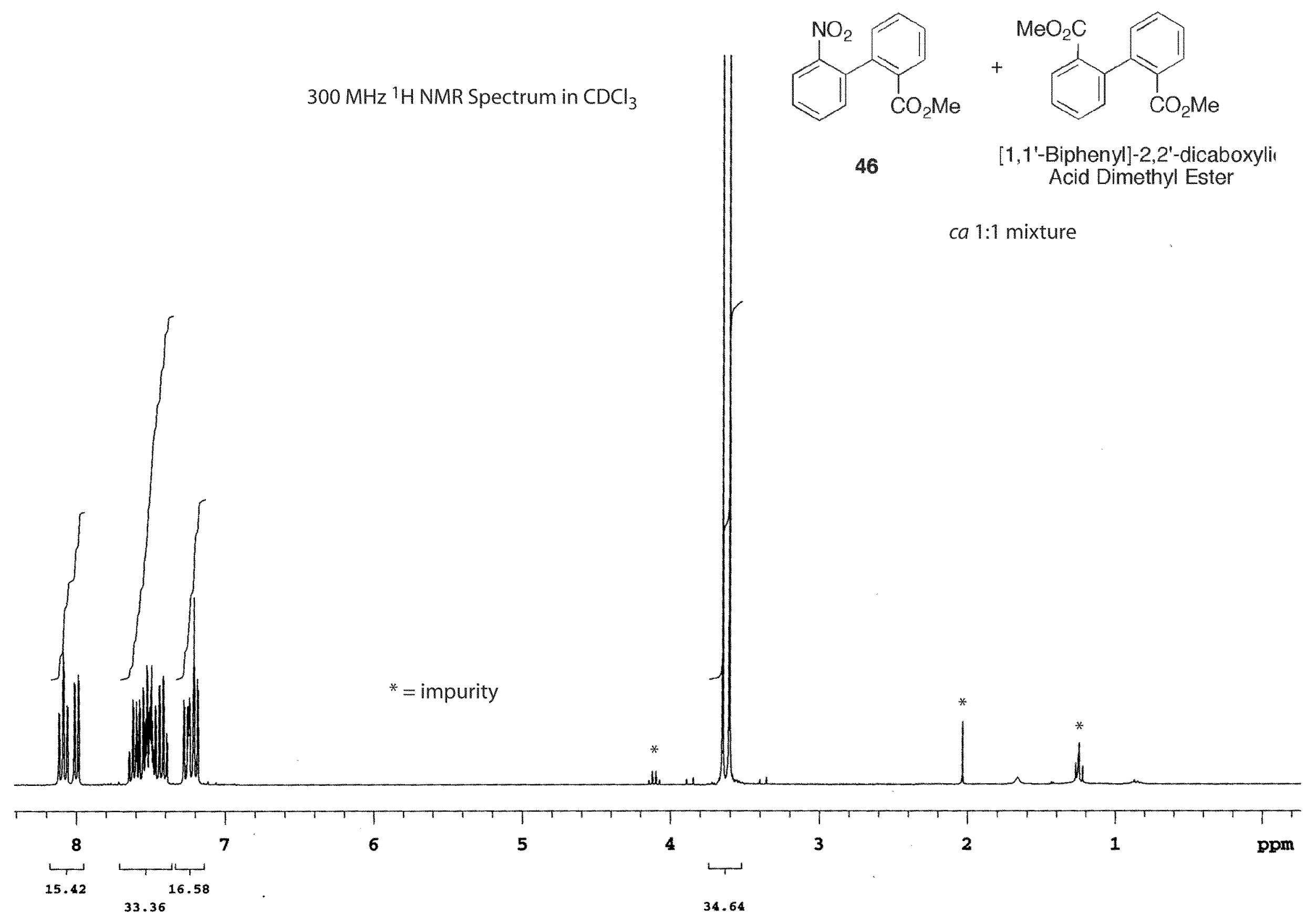




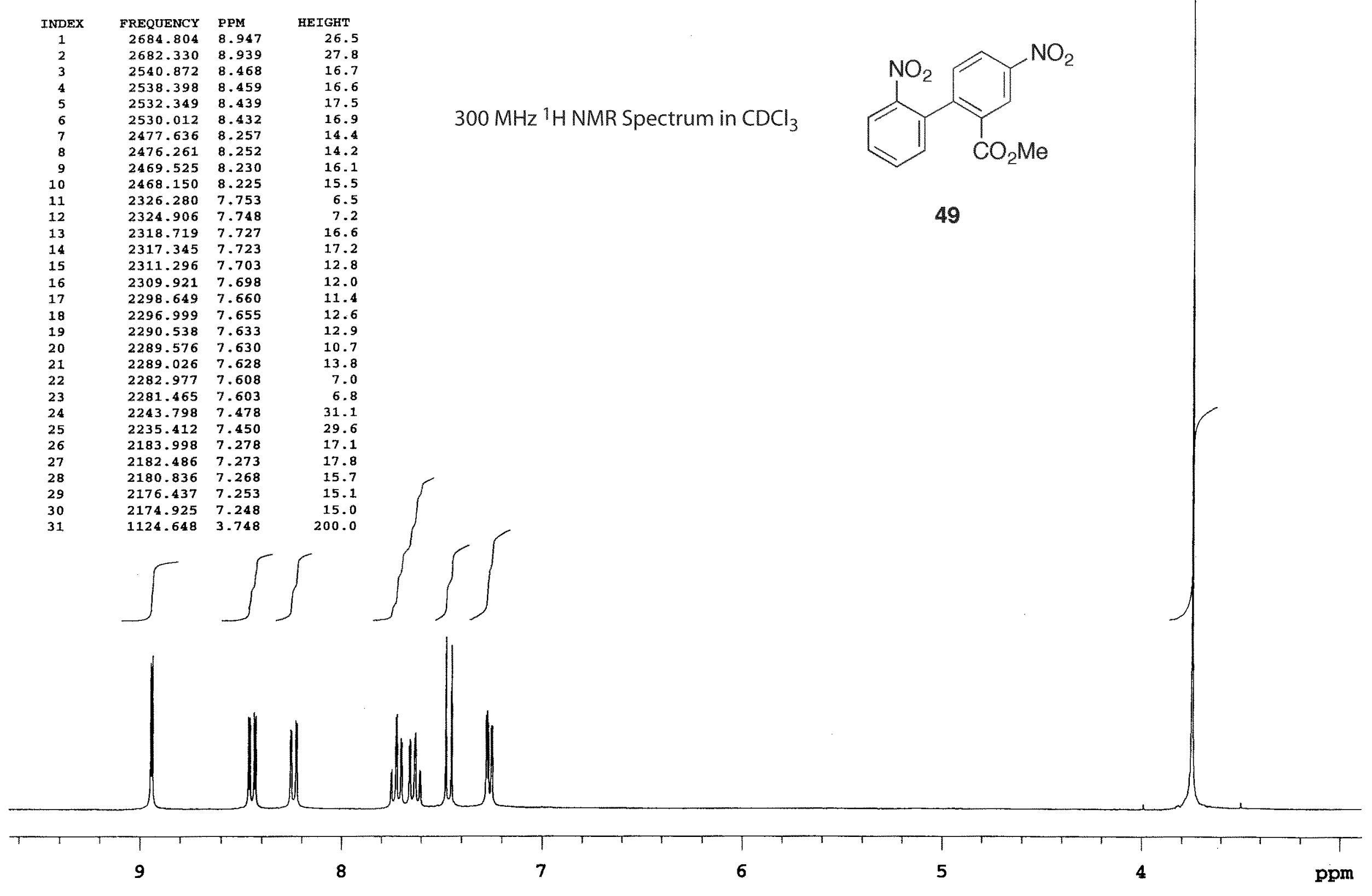




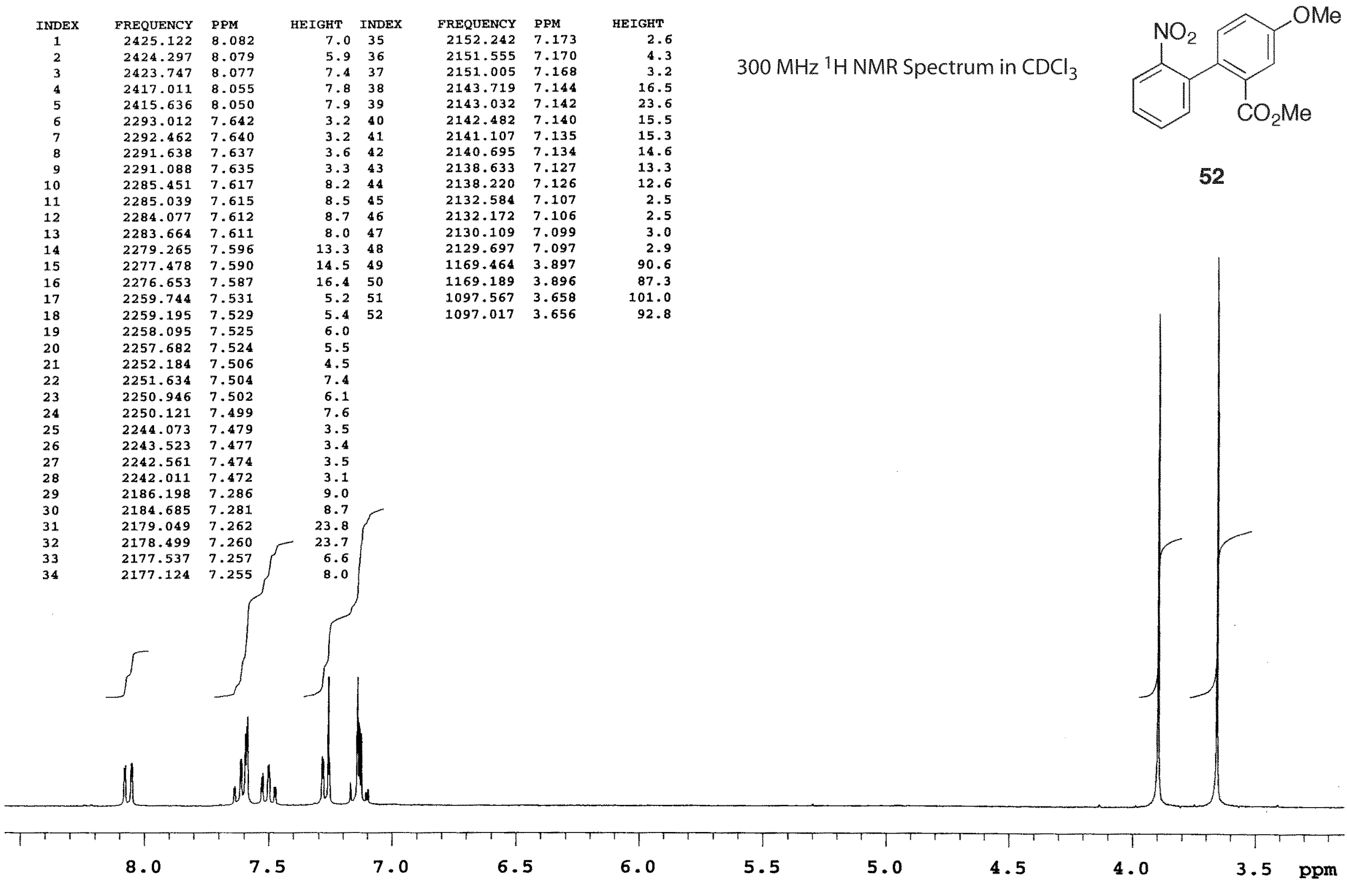




$\begin{array}{crlr}\text { INDEX } & \text { FREQUENCY } & \text { PPM } & \text { HEIGHT } \\ 1 & 2452.319 & 8.173 & 11.1 \\ 2 & 2451.082 & 8.168 & 11.8 \\ 3 & 248.608 & 8.160 & 4.5 \\ 4 & 2444.209 & 8.146 & 15.0 \\ 5 & 244.834 & 8.141 & 18.9 \\ 6 & 2441.322 & 8.136 & 48.9 \\ 7 & 2440.360 & 8.133 & 41.1 \\ 8 & 2375.748 & 7.917 & 14.4 \\ 9 & 2374.786 & 7.914 & 21.4 \\ 10 & 2373.686 & 7.911 & 13.8 \\ 11 & 2307.700 & 7.691 & 5.2 \\ 12 & 2306.326 & 7.686 & 5.5 \\ 13 & 2300.139 & 7.665 & 12.4 \\ 14 & 2298.765 & 7.661 & 12.2 \\ 15 & 2292.716 & 7.641 & 9.3 \\ 16 & 2291.341 & 7.636 & 8.6 \\ 17 & 2276.219 & 7.586 & 8.4 \\ 18 & 2274.707 & 7.581 & 9.1 \\ 19 & 2268.109 & 7.559 & 9.6 \\ 20 & 2267.009 & 7.555 & 9.9 \\ 21 & 2260.685 & 7.534 & 5.6 \\ 22 & 2259.173 & 7.529 & 5.2 \\ 23 & 2189.888 & 7.298 & 12.1 \\ 24 & 2188.376 & 7.293 & 12.3 \\ 25 & 2182.464 & 7.273 & 10.9 \\ 26 & 2180.815 & 7.268 & 10.8 \\ 27 & 2178.478 & 7.260 & 7.1 \\ 28 & 1178.928 & 3.929 & 122.8 \\ 29 & 1104.281 & 3.680 & 123.4 \\ & & & \end{array}$

$300 \mathrm{MHz}{ }^{1} \mathrm{H} \mathrm{NMR} \mathrm{Spectrum} \mathrm{in}\left(\mathrm{CD}_{3}\right)_{2} \mathrm{SO}$

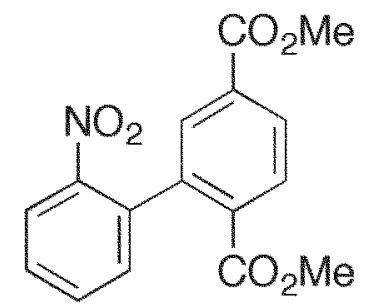

55

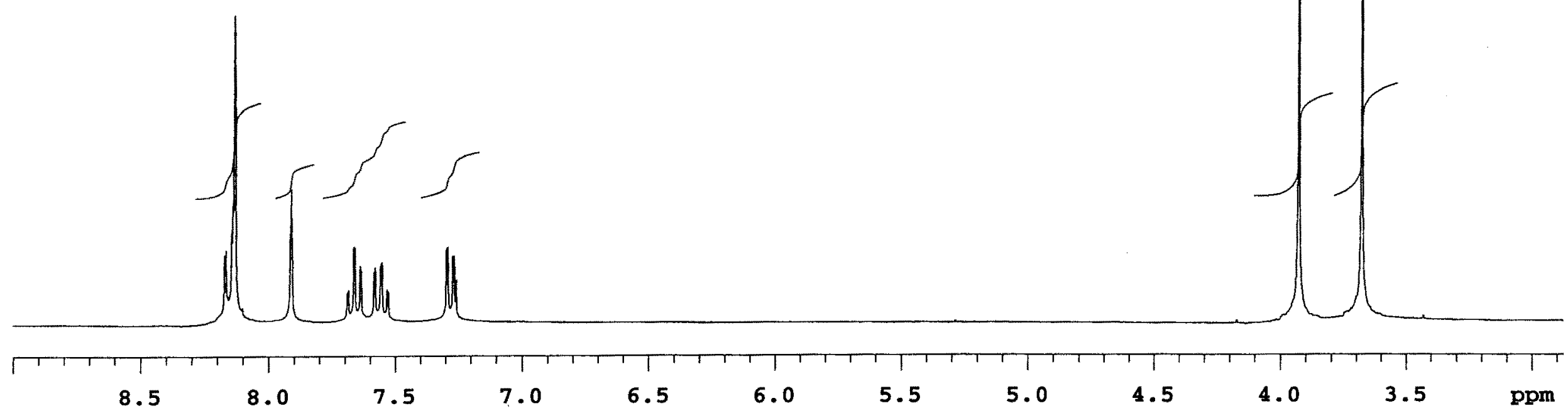


FREQUENCY PPM HEIGHT

$\begin{array}{lll}3567.219 & 11.888 & 16 .\end{array}$

$2690.155 \quad 8.965$

$2534.676 \quad 8.447$

$2526.428 \quad 8.420$

$2495.359 \quad 8.316$

$2494.260 \quad 8.312$

$2444.495 \quad 8.146$

$2436.110 \quad 8.119$

$2270.045 \quad 7.565$

$2261.934 \quad 7.538$

13.269 7.376

$\begin{array}{lll}2198.697 & 7.327\end{array}$

$\begin{array}{lll}2191.686 & 7.304\end{array}$

$\begin{array}{lll}2183.576 & 7.277\end{array}$

$1188.287 \quad 3.960$

38.3

44. 0

49.7

51.1

50.6

24.9

21.2

$300 \mathrm{MHz}{ }^{1} \mathrm{H}$ NMR Spectrum in $\left(\mathrm{CD}_{3}\right)_{2} \mathrm{SO}$

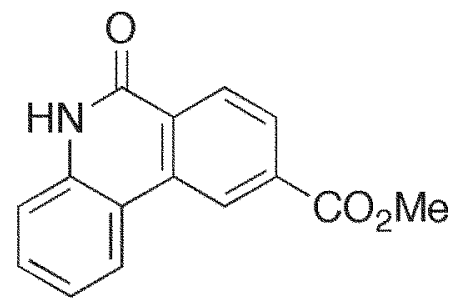

56

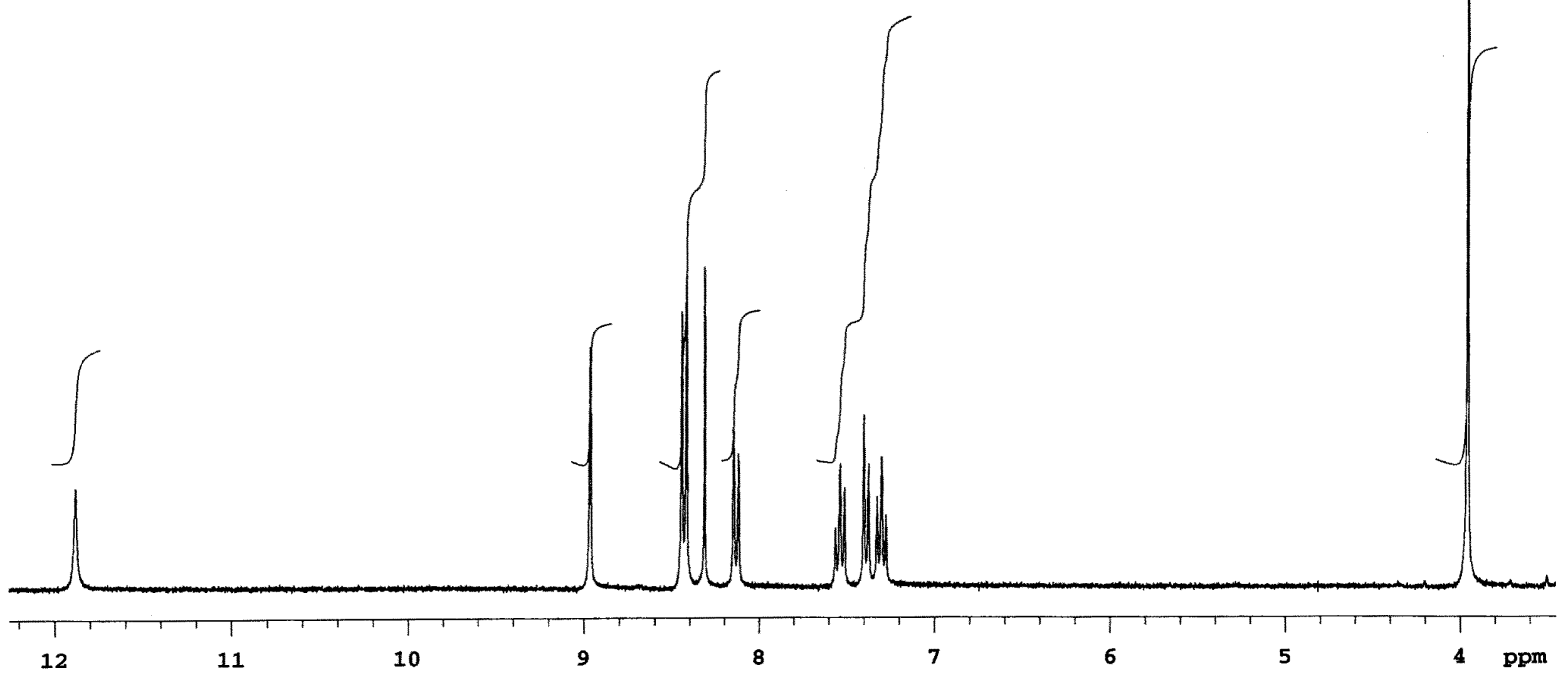


$300 \mathrm{MHz}^{1} \mathrm{H}$ NMR Spectrum in $\mathrm{CDCl}_{3}$

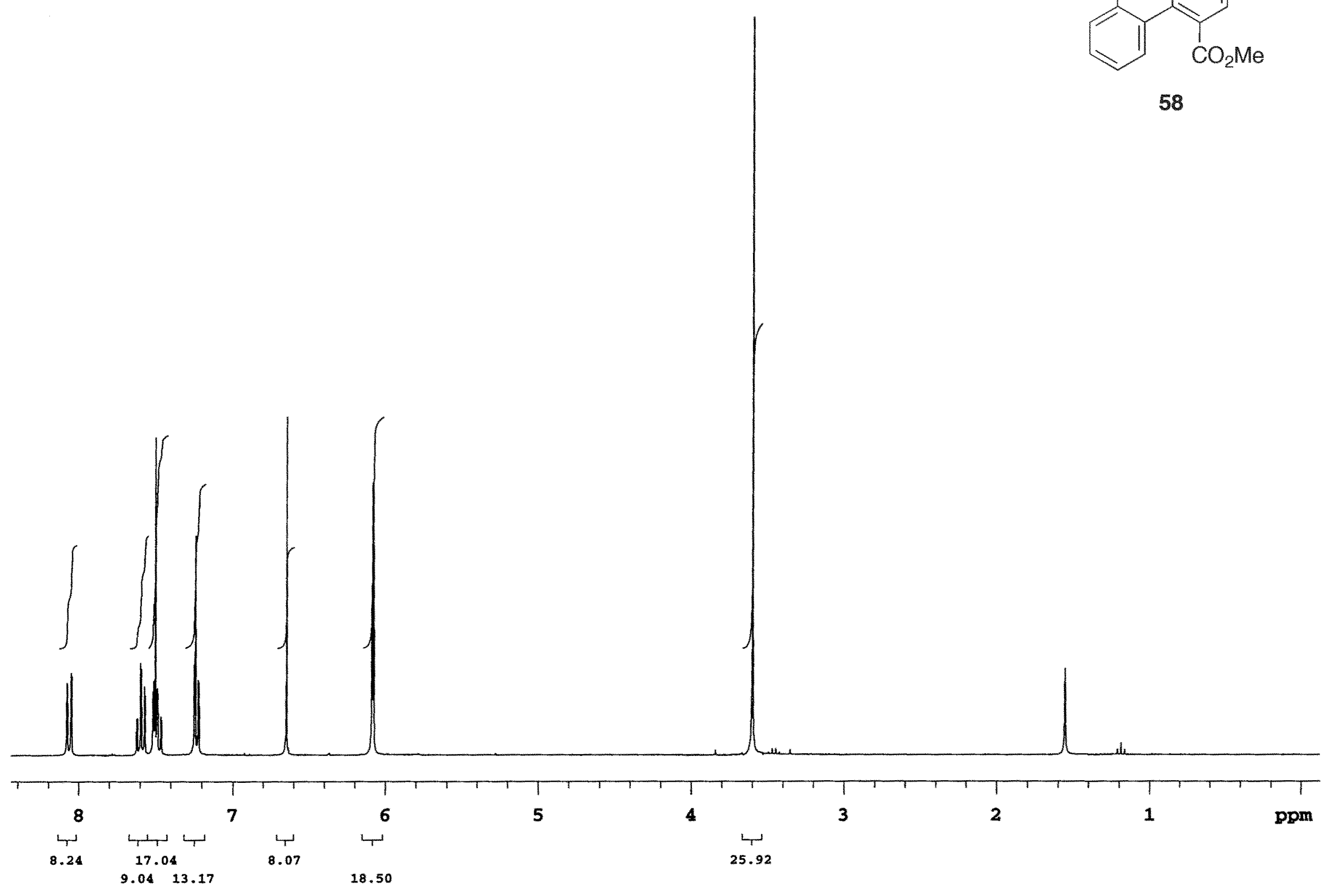

